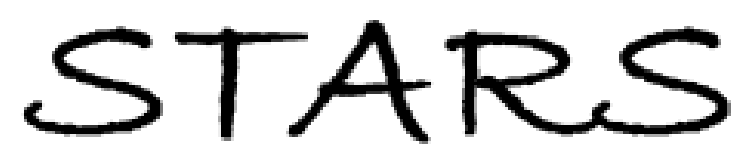

University of Central Florida

STARS

\title{
Unified Steady-state Computer Aided Model For Soft-switching DC-DC Converters
}

\author{
Wisam Al-Hoor \\ University of Central Florida
}

Part of the Electrical and Electronics Commons

Find similar works at: https://stars.library.ucf.edu/etd

University of Central Florida Libraries http://library.ucf.edu

This Masters Thesis (Open Access) is brought to you for free and open access by STARS. It has been accepted for inclusion in Electronic Theses and Dissertations, 2004-2019 by an authorized administrator of STARS. For more information, please contact STARS@ucf.edu.

\section{STARS Citation}

Al-Hoor, Wisam, "Unified Steady-state Computer Aided Model For Soft-switching DC-DC Converters" (2006). Electronic Theses and Dissertations, 2004-2019. 835.

https://stars.library.ucf.edu/etd/835

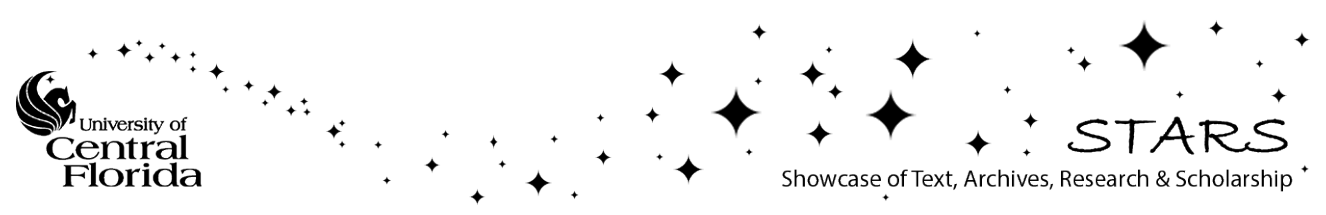




\title{
UNIFIED STEADY-STATE COMPUTER AIDED MODEL FOR SOFT-SWITCHING DC-DC CONVERTERS
}

\author{
By \\ WISAM AL-HOOR \\ B.Sc. Princess Sumaya University for Technology, 2002
}

\begin{abstract}
A thesis submitted in partial fulfillment of the requirements for the degree of Master of Science

in the School of Electrical Engineering and Computer Science

in the College of Engineering and Computer Science at the University of Central Florida

Orlando, Florida
\end{abstract}

Spring Term

2006 
(C) 2006 Wisam Al-Hoor 


\begin{abstract}
For many decades, engineers and students have heavily depended on simulation packages such as Pspice to run transit and steady-state simulation for their circuits. The majority of these circuits, such as soft switching cells, contain complicated modes of operations that require the Pspice simulation to run for a long time and, finally, it may not reach a convergent solution for these kinds of circuits. Also, there is a need for an educational tool that provides students with a better understanding of circuit modes of operation through state-plan figures and steady-state switching waveforms.

The unified steady-state computer aided model proposes a simulation block that covers common unified soft-switching cells operations and can be used in topologies simulation. The simulation block has a simple interface that enables the user to choose the switching cell type and connects the developed simulation model in the desired topology configuration. In addition to the measured information that can be obtained from the circuitry around the unified simulation model, the simulation block includes some additional nodes (other than the inputs and outputs) that make internal switching cell information, such as switching voltages and currents, easy to access and debug. The model is based on mathematical equations, resulting in faster simulation times, smaller file size and greatly minimized simulation convergence problems.
\end{abstract}


The Unified Model is based on the generalized analysis: Chapter 1 discusses the generalized equation concept along with a detailed generalization example of one switching cell, which is the zero current switching quasi-resonant converter ZCS-QRC. Chapter 2 presents a detailed discussion of the unified model concept, the unified model flow chart and the unified model implementation in Pspice. Chapter 3 presents the unified model applications; generating the switching cell inductor current $I_{L r}$ and the switching cell capacitor voltage $V_{C r}$ steady-state waveforms, the State-Plane Diagram, the feedback design using the unified model, and the chapter concludes with how the model can be used with different topologies. Finally, chapter 4 presents the summary and the future work. 
To my parents with love and gratitude 


\section{ACKNOWLEDGMENTS}

First and foremost, all the praises and thanks are to Allah for his persistent blessings. Then, I would like to thank my supervisor Dr. Issa Batarseh for his assistance, support and for giving me the opportunity to pursue my higher education. I would like also to thank Dr. Jaber Abu-Qahouq for his encouragement and thought provoking ideas that helped me in this thesis work. Finally, I would like to express my sincere love and gratitude to my parents and siblings whose love, support and encouragement have been the root of this success. 


\section{TABLE OF CONTENTS}

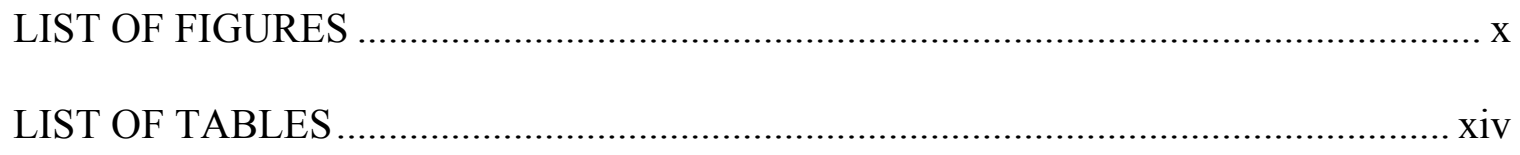

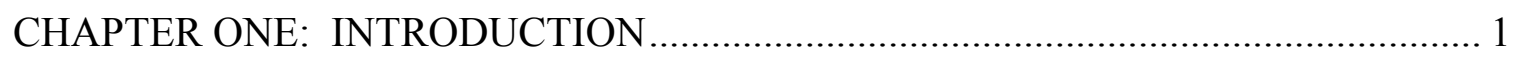

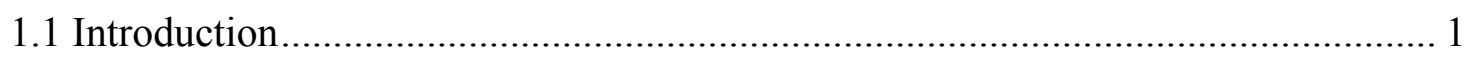

1.2 The Generalized switching cells and their parameters .................................. 4

1.3 The generalized Transformation Table ........................................................ 8

1.4 ZCS-QRC Generalized Switching Cell and derived family .............................. 13

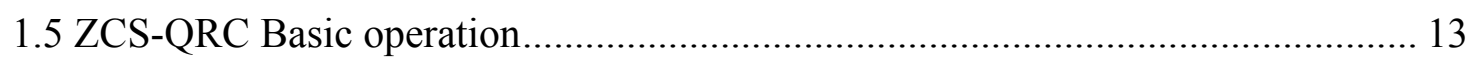

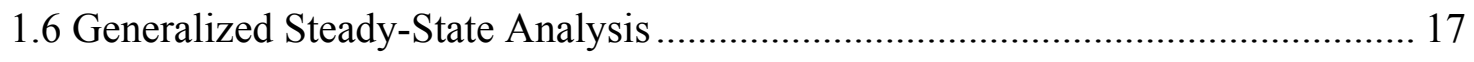

1.6.1 Generalized Intervals Equations ............................................... 17

1.6.2 Generalized Gain Equation ...................................................... 19

1.6.3 Generalized ZCS Condition ........................................................ 20

1.6.4 Generalized peak resonant Inductor Current and capacitor voltage .... 21

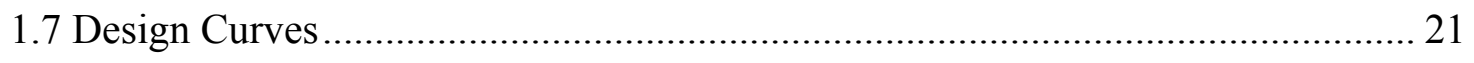


2.1 Introduction. 24

2.2 Unified Steady-State Model Flow-chart ......................................................... 25

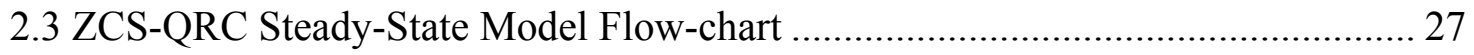

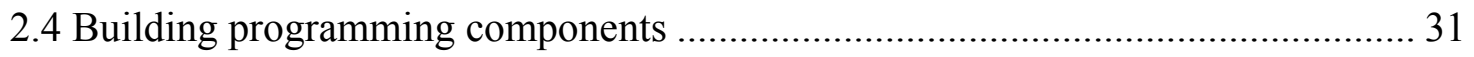

2.4.1 Creating variables in Pspice...................................................... 31

2.4.2 Creating Loops in Pspice ......................................................... 32

2.4.2.1 Creating FOR Loop........................................................... 33

2.4.2.2 Creating WHILE Loop ......................................................... 34

2.5 Developing System Architecture ................................................................ 35

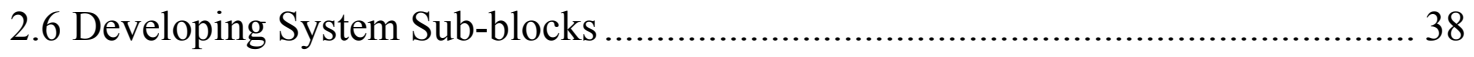

2.6.1 Unified model Inputs ............................................................ 38

2.6.2 Normalized Switching Frequency Block ..................................... 39

2.6.3 Quality Factor \& Characteristics Impedance Block “QZ Block”....... 43

2.6.4 Beta, Gamma and Alpha Block “BG Block”.................................. 47

2.6.4.1 Building Inverse Sine function in Pspice................................. 51

2.6.5 Error Block “ERR Block”, ................................................................. 56

2.6.6 Steady-State Gain Solution Block "SOL Block”.............................. 59

2.6.6.1 FOR Loop with Breakout capability...................................... 60 


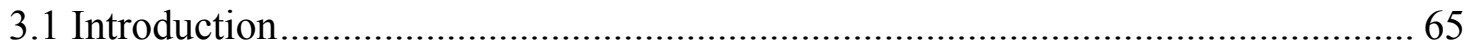

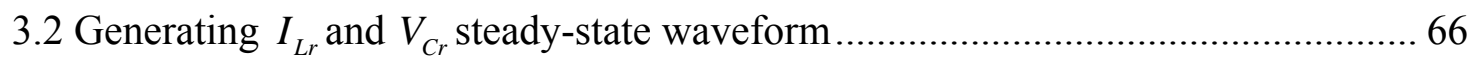

3.3 Generating the State-Plane Diagram........................................................... 75

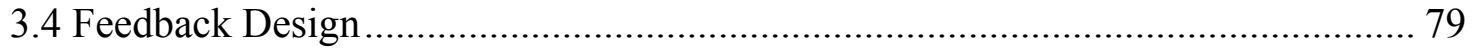

3.4.1 Detecting changes in switching frequency .................................. 80

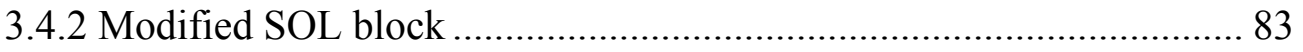

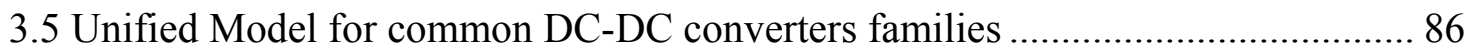

3.5.1 Unified Model for Boost converter family ................................................. 86

3.5.2 Unified Model for Buck-Boost converter family........................................ 89

3.5.3 Selecting between different topologies ................................................... 91

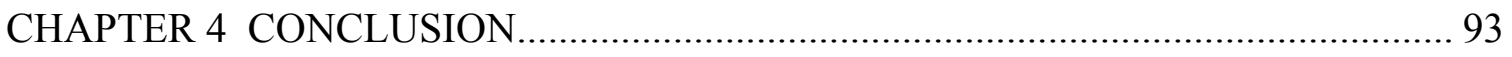

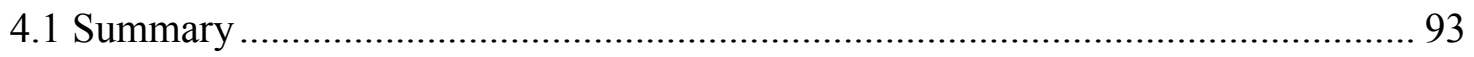

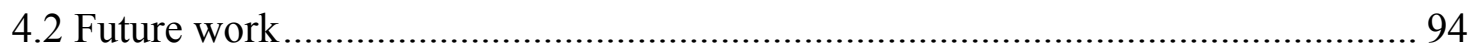

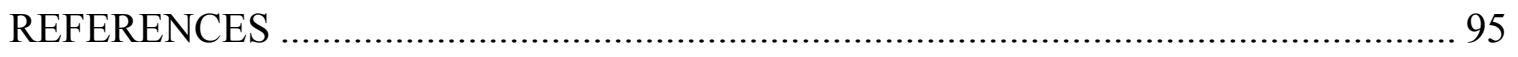




\section{LIST OF FIGURES}

Figure 1.1: Switching-Cells: (a) Conventional Cell ………....................................... 7

Figure 1.1: Switching-Cells: (b) ZVS-QRC Cell....................................................... 7

Figure 1.1: Switching-Cells: (c) ZCS-QRC Cell ......................................................... 7

Figure 1.1: Switching-Cells: (d) ZVS-QSW CV Cell .................................................... 7

Figure 1.1: Switching-Cells: (e) ZCS-QSW CC Cell, ............................................... 7

Figure 1.1: Switching-Cells: (f) ZVT-PWM Cell.......................................................... 7

Figure 1.1: Switching-Cells: (g) ZCT-PWM Cell ………............................................. 7

Figure 1.2: The Conventional DC-DC Converters: (a) Buck ……………...................... 12

Figure 1.2: The Conventional DC-DC Converters: (b) Boost .......................................... 12

Figure 1.2: The Conventional DC-DC Converters: (c) Buck-Boost............................... 12

Figure 1.2: The Conventional DC-DC Converters: (d) Cuk.......................................... 12

Figure 1.2: The Conventional DC-DC Converters: (e) Zeta............................................ 12

Figure 1.2: The Conventional DC-DC Converters: (f) Sepic ......................................... 12

Figure 1.3: The Generalized ZCS-QRC Switching Cell with Uni-directional Switch ..... 13

Figure 1.4: The DC-DC ZCS-QRC Family:(a) Buck ……………………………....... 15

Figure 1.4: The DC-DC ZCS-QRC Family: (b) Boost............................................... 15

Figure 1.4: The DC-DC ZCS-QRC Family: (c) Buck-Boost ......................................... 15

Figure 1.4: The DC-DC ZCS-QRC Family: (d) Cuk ................................................ 15

Figure 1.4: The DC-DC ZCS-QRC Family: (e) Zeta ……………................................ 15

Figure 1.4: The DC-DC ZCS-QRC Family: (f) Sepic .................................................. 15 
Figure 1.5: Main ZCS-QRC switching cell waveforms................................................. 16

Figure 1.6: The equivalent circuits for: (a) Mode 1 _................................................ 19

Figure 1.6: The equivalent circuits for: (b) Mode 2 ………..................................... 19

Figure 1.6: The equivalent circuits for: (c) Mode 3 …………..................................... 19

Figure 1.6: The equivalent circuits for: (d) Mode 4................................................... 19

Figure 1.7a: DC voltage conversion ratio characteristics for ZCS-QRC Buck ................ 22

Figure 1.7b: DC voltage conversion ratio characteristics for ZCS-QRC Boost............... 22

Figure 1.7c: DC voltage conversion ratio characteristics for ZCS-QRC Buck-Boost, Cuk,

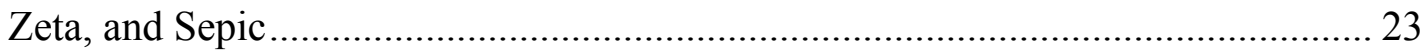

Figure 1.7d: DC voltage conversion ratio characteristics for The Minimum Allowed Duty

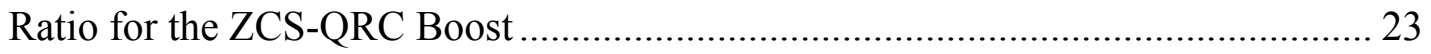

Figure 2.1: Unified Steady-State Model Flow-chart........................................................ 26

Figure 2.2: ZCS-QRC buck unified equation solution Flow-chart.................................. 29

Figure 2.3: ZCS-QRC buck steady-state waveforms using MATLAB ……………....... 30

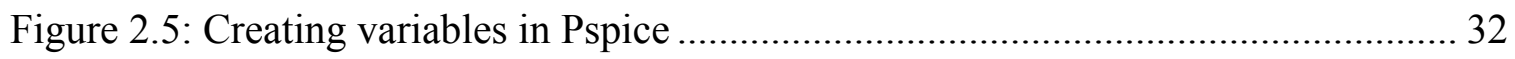

Figure 2.6: FOR loop circuit in Pspice …………………....................................... 33

Figure 2.7: WHILE loop circuit in Pspice ................................................................... 34

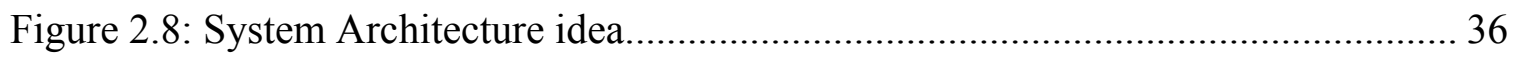

Figure 2.9: System Architecture Implementation in Pspice ........................................... 37

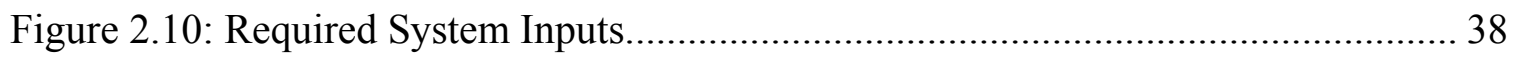

Figure 2.11: Normalized Switching Frequency Block …………………….................... 39

Figure 2.12: Normalized Switching Frequency Block flowchart .................................... 41

Figure 2.13: Pspice implementation of $f_{n s}$ Block ...................................................... 41 
Figure 2.14: Pspice Simulation results for $f_{n s}$ Block..................................................... 42

Figure 2.15: Quality Factor \& Characteristics Impedance Block................................... 43

Figure 2.16: Quality Factor and the Characteristics Impedance $Q Z$ block flowchart.... 45

Figure 2.17: Pspice implementation of QZ Block ......................................................... 46

Figure 2.18: Pspice Simulation results for $f_{n s}$ Block.................................................... 47

Figure 2.19: Alpha, Beta and Gamma Block .............................................................. 47

Figure 2.20: Alpha, Beta and Gamma block flowchart .................................................. 50

Figure 2.21: Maclaurin series approximation versus actual $S N^{-1}$ function ..................... 53

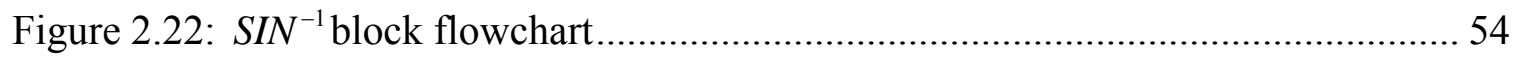

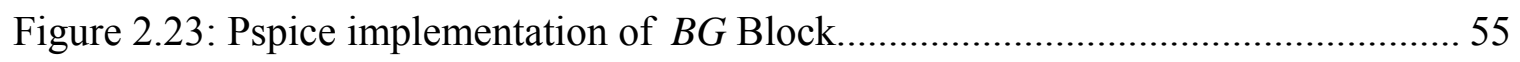

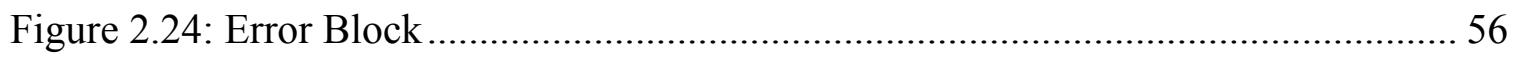

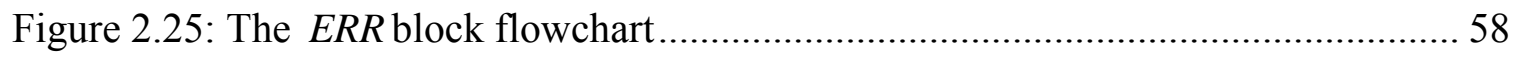

Figure 2.26: Pspice implementation of the ERR Block ................................................. 59

Figure 2.27: The Steady-State Gain Solution Block..................................................... 59

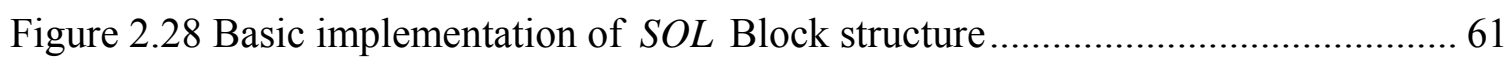

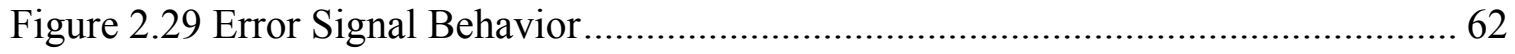

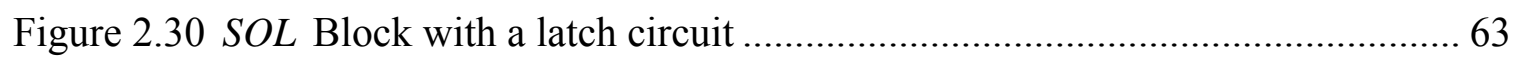

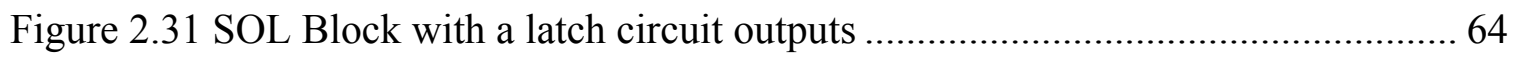

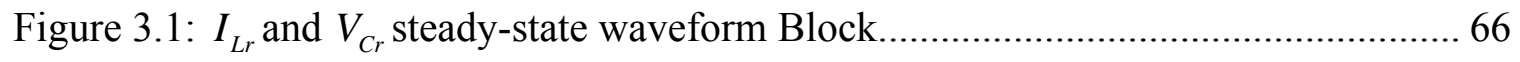

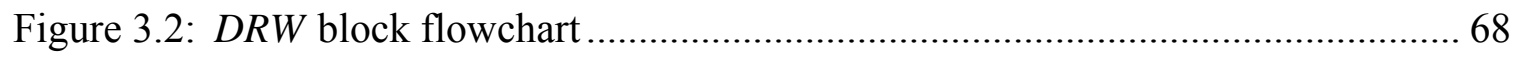

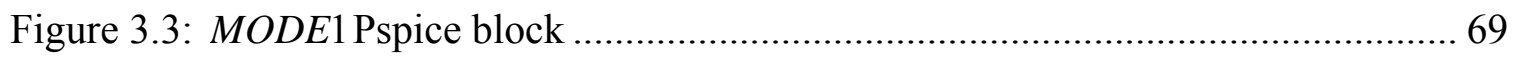

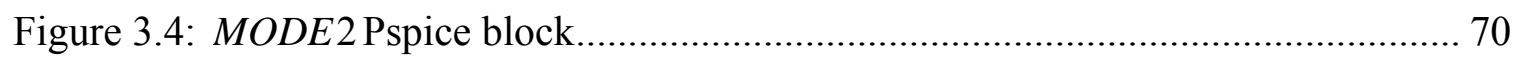

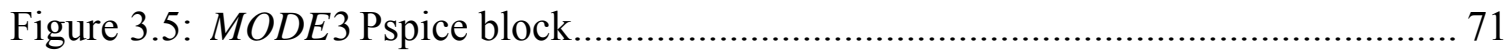




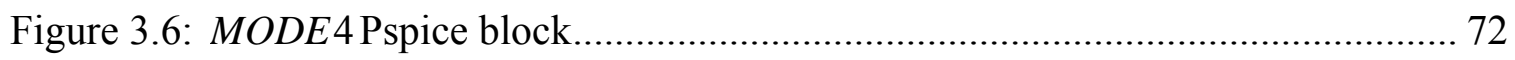

Figure 3.7: All Modes summation Pspice block …………….......................................... 73

Figure 3.8: Finding time intervals Pspice block ........................................................... 74

Figure 3.9: $I_{L r}$ and $V_{C r}$ steady-state waveform ....................................................... 74

Figure 3.10: The Generalized State plane for ZCS-QRC Switching Cell ....................... 75

Figure 3.11: Switching cell state-plane diagram........................................................ 78

Figure 3.12: Closed loop using the unified general model ............................................. 79

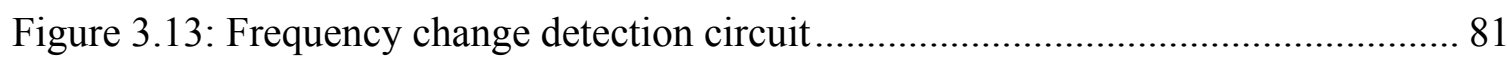

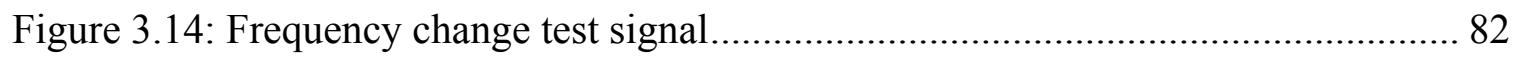

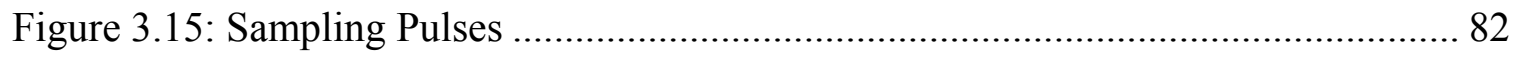

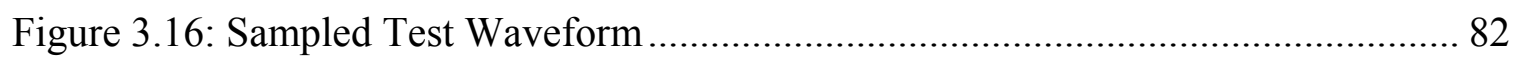

Figure 3.17 Frequency change detection block output result ......................................... 83

Figure 3.18: Pspice implementation of counter idea ………………………………...... 84

Figure 3.19 modified capacitor-counter circuit .......................................................... 85

Figure 3.20 Unified Model step response ………………........................................ 85

Figure 3.21 Error block for the Boost topology ……………...................................... 88

Figure 3.22 Error block for the Buck-Boost topology ……............................................ 91

Figure 3.23 Complete Error block with topology selection............................................. 92 


\section{LIST OF TABLES}

Table 1.1: Generalized Transformation Table ................................................................ 8

Table 2.1: Generalized Transformation Table ............................................................. 28

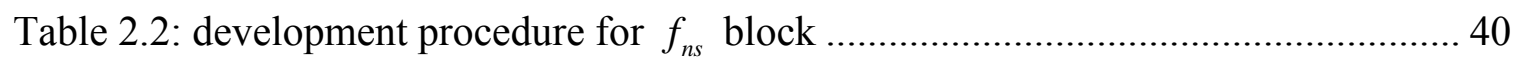

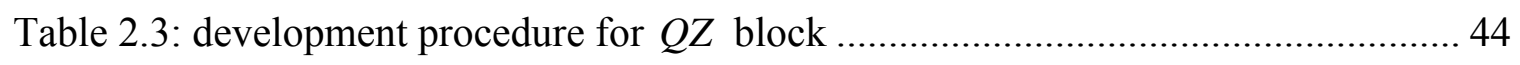

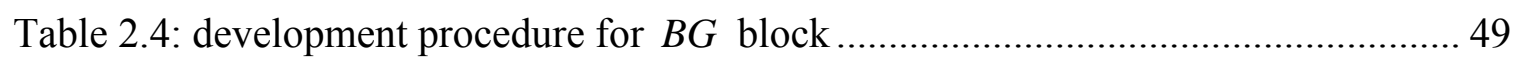

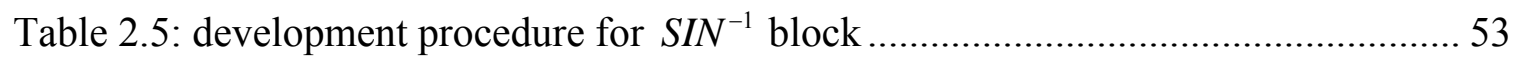

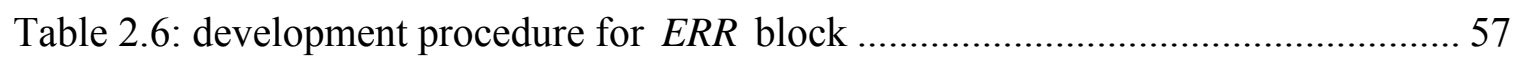

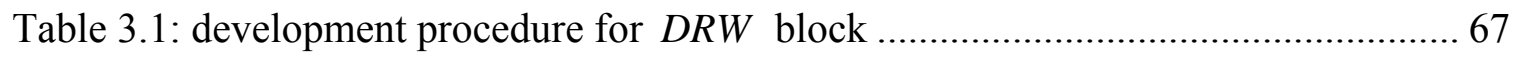

Table 3.2: Comparison between theoretical values and Model calculated values........... 79 


\section{CHAPTER ONE INTRODUCTION}

\subsection{Introduction}

The ever increasing demand for DC-DC converters with high power density and high efficiency introduces many technologies to the power converters industry. One of those technologies is different soft-switching techniques [1-10]. The main idea behind soft-switching is to create a resonant condition across the converter main switches to force the voltage across the switch to decrease to zero before the switch turns ON in what is knows as Zero Voltage Switching (ZVS), or to force the current passing through the switch to decrease to zero before the switch turns OFF in what is known as Zero Current Switching (ZCS).

Soft-switching techniques add many advantages to DC-DC power converters, the most important is the reduction of switching losses and hence the ability to operate at higher switching frequencies. Larger switching frequency means smaller magnetic components size and higher power densities [1-5]. Soft-switching cells achieve those advantages by employing additional resonant components and auxiliary switches and diodes. 
Designing soft-switching cells requires a thorough understanding of the cell properties and operation, and this in turn requires accurate analysis of the cell during different main and transition modes of operations at one switching cycle. This is a difficult and time consuming process, especially when considering the added resonant components and auxiliary switches and diodes $[9,10]$.

A simpler and a faster way to analyze and find a steady-state solution for softswitching cells is by using generalized analysis. Generalized analysis is based on the idea that soft-switching cells will have the same modes of operation and the same switching waveforms in spite of cell orientation. Therefore, instead of analyzing switching cells contained in different converters as a one topology, only the switching cell needs to be analyzed and the result can be applied to different DC-DC converter families using transformation tables [2].

In this thesis and based on the generalized equations, a unified simulation model is derived. The unified steady-state simulation model is a simulation block that covers common unified soft-switching cell operations [1-16], with defined inputs and outputs, and can be used in topologies simulation. The user can choose the switching cell type and can connect the developed simulation model in the desired topology configuration. In addition to the measurement information that can be obtained from the circuitry around the unified simulation model, the simulation block includes some additional nodes (other than the inputs and outputs) that give internal switching cell information, such as switching voltages and currents. The models is based on mathematical equations, which 
results in faster simulation times, smaller file size, and greatly minimizes simulation convergence problems.

The proposed unified simulation model is implemented in Pspice/Orcad $\mathbb{}$ simulation software $[17,18]$ to provide a model that can be used as a part of complete simulation schematic that may include other models in Pspice/Orcad $\AA$. Using the mathematical equations approach to implement the model requires the ability to implement programming loops and perform instructions in certain sequence. This was the main challenge in this work because Pspice/Orcad ${ }^{\circledR}$ is not a programming language and soft -switching cells have complicated set of equations that describe the operation modes.

Chapter 1 generally discusses the generalized equation concept, section 1.2 introduces the switching cell parameters, and section 1.3 shows the generalized transformation table derivation. To introduce the proposed unified model development and related development issues and challenges, the zero current switching (ZCS) quasiresonant $(\mathrm{QRC})$ unified switching cell $[2,3,9]$ was selected. In zero current switching families, the switches are turned OFF at zero current. To achieve ZCS, the soft-switching cell utilizes circuit non-idealities like the transformer leakage inductance, junction diode capacitance and the power switch parasitic capacitance. Chapter 1 gives a complete analysis of the ZCS-QRC family beginning in Section 1.4 by presenting the ZCS-QRC Generalized switching cell and it is derived family. Section 1.5 introduces the basic operation of the ZCS-QRC along with its modes of operation and generalized switching waveforms, followed by a discussion of the generalized steady-state analysis in section 1.6. Finally section 1.7 shows some design curves for the ZCS-QRC family. 


\subsection{The Generalized switching cells and their parameters}

Figure 1.1 shows the generalized switching cells for number of selected families, it can be noted that those cells have the same modes of operations and thus their analysis can be generalized. Different converters analysis can be obtained by using different orientation of any of the cells in Figure 1.1. Using the three terminal notations $a, b$ and $c$ for the switching cells, the following generalized parameters can be defined as follow:

- The over-all output to input converter gain (M):

$$
M=\frac{V_{o}}{V_{\text {in }}}=\frac{I_{\text {in }}}{I_{o}}
$$

Where :

$V_{\text {in }}$ is the converter average input voltage.

$V_{o}$ is the converter average output voltage.

$I_{\text {in }}$ is the converter average input current.

$I_{o}$ is the converter average output current.

- The normalized cell input voltage $\left(V_{n g}\right)$ :

$$
V_{n g}=\frac{V_{g}}{V_{i n}}
$$

Where $V_{g}$ is the switching-cell average input voltage as show in Figure 1.1. 
- The normalized cell output current $\left(I_{n F}\right)$ :

$$
I_{n F}=\frac{I_{F}}{I_{o}}
$$

Where $I_{F}$ is the switching-cell average output current as shown in figure 1.1.

- The normalized filter capacitor voltage $\left(V_{n F}\right)$ :

$$
V_{n F}=\frac{V_{F}}{V_{i n}}
$$

Where $V_{F}$ is the filter capacitor average voltage.

- The normalized filter inductor current $\left(I_{n T}\right)$ :

$$
I_{n T}=\frac{I_{T}}{I_{o}}
$$

Where $I_{T}$ is the filter inductor average current.

- The normalized cell output average voltage $\left(V_{n b c}\right)$

$$
V_{n b c}=\frac{V_{b c}}{V_{i n}}
$$

Where $V_{b c}$ is the switching cell average output voltage.

- The normalized current entering node $\mathrm{b}$ in the switching cell $\left(I_{n b}\right)$ :

$$
I_{n b}=\frac{I_{b}}{I_{o}}
$$

Where $I_{b}$ is the average current entering node $b$. 
- The resonant frequency $\left(f_{o}\right)$ in $\mathrm{Hz}$ :

$$
f_{o}=\frac{1}{2 \pi \sqrt{C_{r} L_{r}}}=\frac{\omega_{o}}{2 \pi}
$$

where:

$\omega_{o}$ is the resonant frequency in $\mathrm{rad} . / \mathrm{sec}$.

$C_{r}$ is the resonant capacitor.

$L_{r}$ is the resonant inductor.

- The switching frequency $\left(f_{s}\right)$ in $\mathrm{Hz}$ :

$$
f_{s}=\frac{1}{T_{s}}
$$

where $T_{s}$ is the switching period in seconds.

- The normalized switching frequency $\left(f_{n s}\right)$ :

$$
f_{n s}=\frac{f_{s}}{f_{o}}
$$

- The characteristic impedance $\left(Z_{o}\right)$ in ohms:

$$
Z_{o}=\sqrt{\frac{L_{r}}{C_{r}}}
$$

- The quality factor or the normalized load (Q):

$$
Q=\frac{R_{o}}{Z_{o}}
$$

Where $R_{o}$ is the load resistance in ohms. 


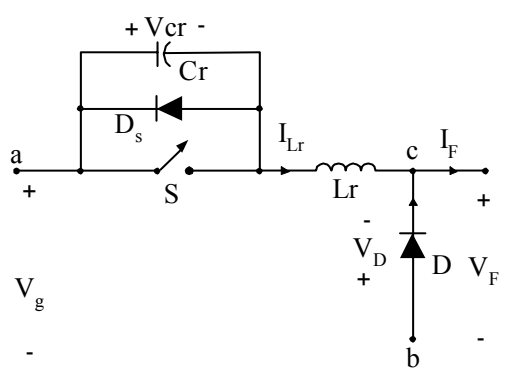

(b)
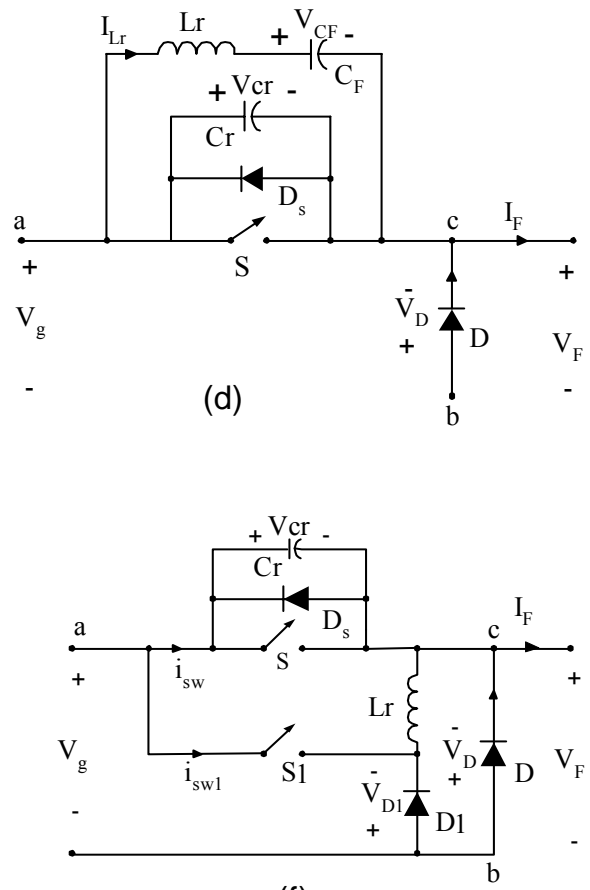

(f)

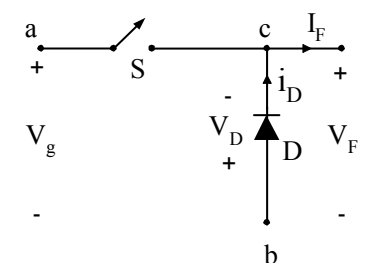

(a)

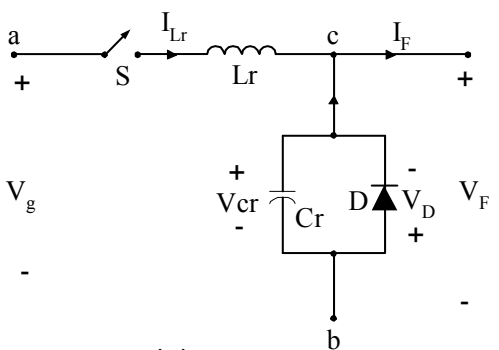

(c)

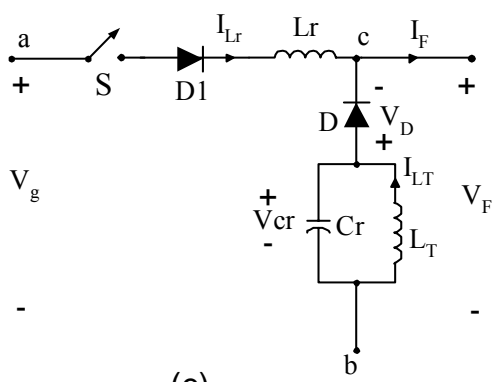

(e)

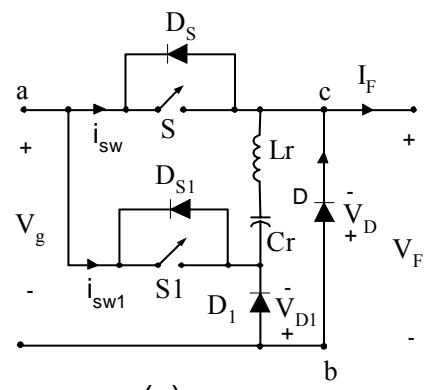

(g)

Figure 1.1: Switching-Cells: (a) Conventional Cell, (b) ZVS-QRC Cell, (c) ZCS-QRC Cell, (d) ZVS-QSW CV Cell, (e) ZCS-QSW CC Cell, (f) ZVT-PWM Cell, and (g) ZCTPWM Cell 


\subsection{The generalized Transformation Table}

A complete set of converters can be obtained by using the various orientations of the generalized three terminal cells shown in Figure 1.1. Figure 1.2 shows the common DC-DC converters, namely, the buck, boost, buck-boost, cuck, zeta, and sepic. The derivation of the generalized transformation for the above converters can be explained in this section by choosing the buck converter as an example. The generalized transformation table is given in Table 1.1. It can be shown that this table is general and can be applied to all cells in Figure 1.1.

Table 1.1: Generalized Transformation Table

\begin{tabular}{|c|c|c|c|}
\hline $\begin{array}{c}\text { Normalized } \\
\text { Parameters }\end{array}$ & Buck & Boost & $\begin{array}{c}\text { Buck -Boost, } \\
\text { Cuk, Zeta, and } \\
\text { Sepic }\end{array}$ \\
\hline$V_{n g}, I_{n F}$ & 1 & $\mathrm{M}$ & $1+\mathrm{M}$ \\
\hline$V_{n F}, I_{n b}, I_{n T}$ & $1-\mathrm{M}$ & 1 & 1 \\
\hline$V_{n b c}$ & $-\mathrm{M}$ & $1-\mathrm{M}$ & $-\mathrm{M}$ \\
\hline
\end{tabular}

The buck converter is shown is part (a) of figure 1.2, the derivation for the generalized parameters for the buck converter will be as following: 
- $V_{n g}$ :

$V_{g}$ is the average voltage between point $a$ and point $b$ in the generalized switching-cells $\left(V_{a b}\right)$. Hence, simply by applying KVL to the buck converter in any of the selected families, $V_{g}$ can be found. This yields to:

$$
V_{g}=V_{a b}=V_{\text {in }}
$$

Normalize $V_{g}$ with respect to $V_{\text {in }}$ :

$$
V_{n g}=\frac{V_{g}}{V_{i n}}=\frac{V_{i n}}{V_{i n}}=1
$$

- $I_{n F}$ :

$I_{F}$ is the average current leaving (going out of) the generalized switching-cell at point $c$. By applying KCL to the buck converter in any of the selected families, $I_{F}$ can be found. This yields to:

$$
I_{F}=I_{c-o u t}=I_{o}
$$

Normalize $I_{F}$ with respect to $I_{o}$ :

$$
I_{n F}=\frac{I_{F}}{I_{o}}=\frac{I_{o}}{I_{o}}=1
$$


- $V_{n b c}$ :

$V_{b c}$ is the average output voltage between node $\mathrm{b}$ and node $\mathrm{c}$ in the generalized switching cell. By applying KVL to the buck converter in any of the selected families, $V_{b c}$ can be found. This yields to:

$$
V_{b c}=-V_{o}
$$

Normalize $V_{F}$ with respect to $V_{i n}$ :

$$
V_{n b c}=\frac{V_{b c}}{V_{i n}}=\frac{-V_{o}}{V_{i n}}=-M
$$

- $V_{n F}$ :

$V_{F}$ is the average voltage across the filter capacitor in the generalized switchingcell. By applying KVL to the buck converter in any of the selected families, $V_{F}$ can be found. This yields to:

$$
V_{F}=V_{i n}-V_{o}
$$

Normalize $V_{F}$ with respect to $V_{\text {in }}$ :

$$
V_{n F}=\frac{V_{F}}{V_{i n}}=\frac{V_{i n}-V_{o}}{V_{i n}}=1-M
$$


- $I_{n b}$ :

$I_{b}$ is the average current entering the generalized switching-cell at point $b$. By applying KCL to the buck converter in any of the selected families, $I_{b}$ can be found. This yields to:

$$
I_{b}=I_{b-i n}=I_{o}-I_{\text {in }}
$$

Normalize $I_{b}$ with respect to $I_{o}$ :

$$
I_{n b}=\frac{I_{b}}{I_{o}}=\frac{I_{o}-I_{i n}}{I_{o}}=1-M
$$

- $I_{n T}$ :

$I_{T}$ is the average current passes through the filter inductor in generalized switching-cell. By applying KCL to the buck converter in Figure 3.9(d), $I_{T}$ can be found. This yields to:

$$
I_{T}=I_{o}-I_{\text {in }}
$$

Normalize $I_{T}$ with respect to $I_{o}$ :

$$
I_{n T}=\frac{I_{T}}{I_{o}}=\frac{I_{o}-I_{i n}}{I_{o}}=1-M
$$

By doing the same derivation for the other converters types, the results will be as shown in Table 1.1. 


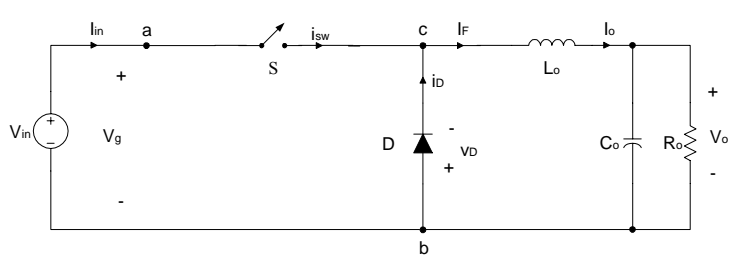

(a)

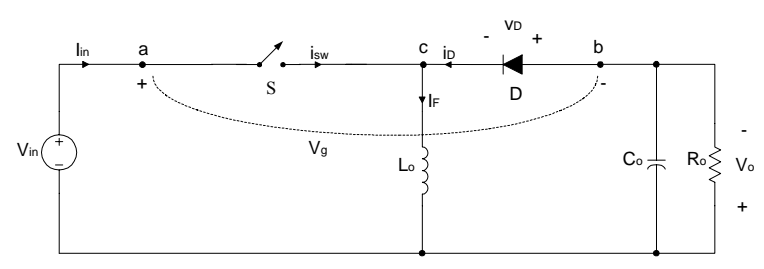

(c)

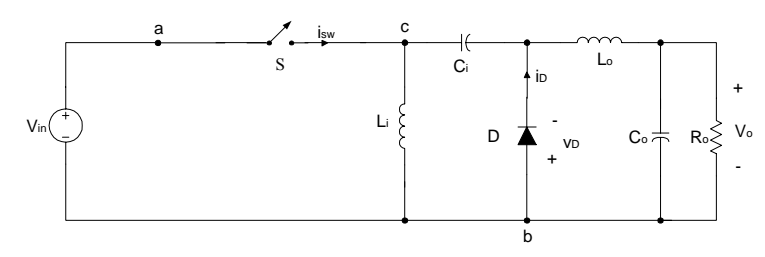

(e)

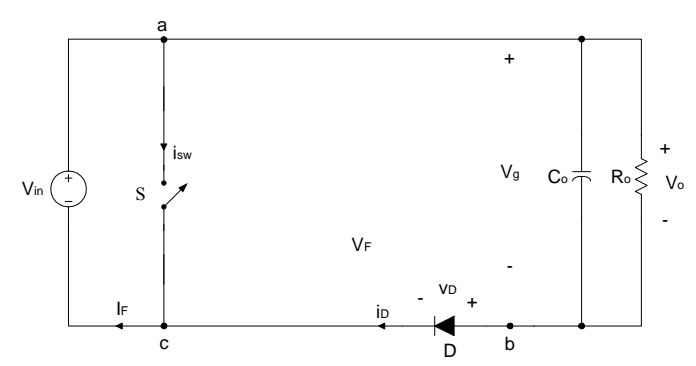

(b)

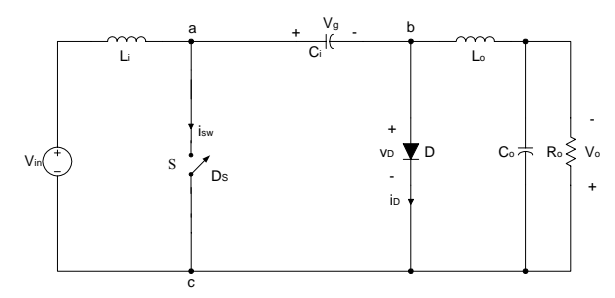

(d)

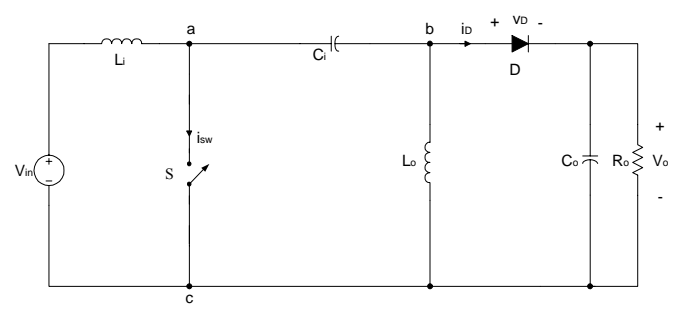

(f)

Figure 1.2: The Conventional DC-DC Converters: (a) Buck, (b) Boost, (c) Buck-Boost, (d) Cuk, (e) Zeta, and (f) Sepic 


\subsection{ZCS-QRC Generalized Switching Cell and derived family}

Figure 1.3 shows the Generalized ZCS-QRC switching cell, by applying this cell to the conventional dc-dc converters in Figure 1.2, the ZCS-QRC family can be formed as shown in Figure 1.4.

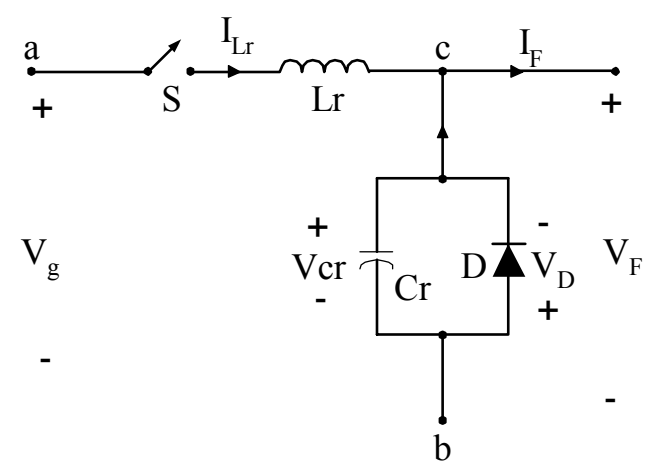

Figure 1.3: The Generalized ZCS-QRC Switching Cell with Uni-directional Switch

In the next section, the ZCS-QRC modes of operation will be discussed and main switching waveforms will be drawn. In this analysis the generalized parameters defined in chapter 1 will be used.

\subsection{ZCS-QRC Basic operation}

The typical switching waveforms for the ZCS-QRC cell in Figure 1.3 are shown in Figure 1.5. It can be shown that there are four modes of operation whose steady-state analysis is summarized as follows: 
It is assumed that before $t=t_{0}, S$ was OFF and $D$ was ON to carry $I_{n F}$. The resonant inductor $L_{r}$ was currying no current and the resonant capacitor $C_{r}$ voltage was zero.

Mode 1: $\left[t_{o} \leq t \leq t_{1}\right]$, starts when $S$ is turned ON while $D$ is ON, which cause $L_{r}$ to charge up linearly until the current through it becomes equal to $I_{n F}$ at $t=t_{1}$ causing $D$ to Turn OFF. The equivalent circuit for this mode is shown in Figure 1.6. The mathematical equation for this mode is as follow (Knowing $v_{C r}\left(t_{0}\right)=0$ and $\left.i_{L r}\left(t_{0}\right)=0\right)$ :

$$
\begin{gathered}
v_{c r}(t)=0 \\
i_{L r}(t)=\frac{V_{g}}{L_{r}}\left(t-t_{0}\right) \\
v_{C r}\left(t_{1}\right)=0 \\
i_{L r}\left(t_{1}\right)=I_{F}
\end{gathered}
$$

Mode 2: $\left[t_{1} \leq t \leq t_{2}\right]$, starts when $D$ turns OFF while $S$ is ON causing a resonant stage between $C_{r}$ and $L_{r}$ to start until the current through $L_{r}$ drops to zero at $t=t_{2}$ causing $S$ to turn OFF at zero current (Soft-switching). The equivalent circuit for this mode is shown in Figure 1.6. The mathematical equation for this mode is as follow: 


$$
\begin{gathered}
v_{C r}(t)=V_{g}\left[1-\cos w_{0}\left(t-t_{1}\right)\right] \\
i_{L r}(t)=I_{F}+\frac{V_{g}}{Z_{o}} \sin w_{0}\left(t-t_{1}\right) \\
i_{L r}\left(t_{2}\right)=0
\end{gathered}
$$

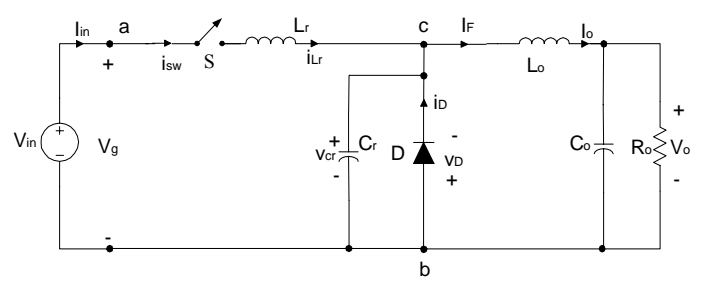

(a)

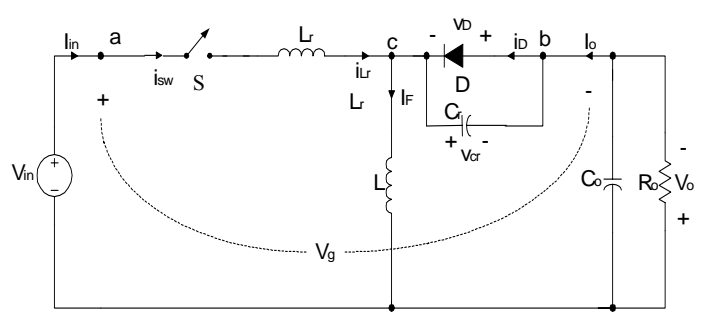

(c)

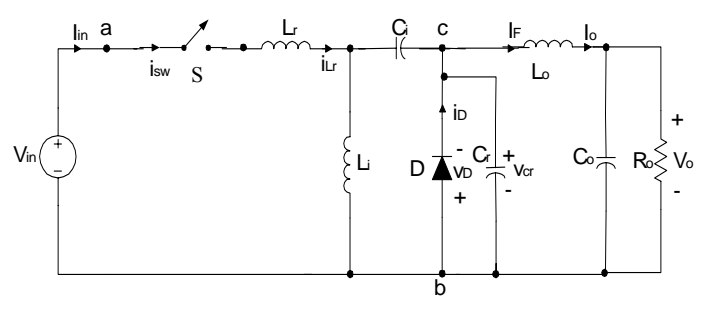

(e)

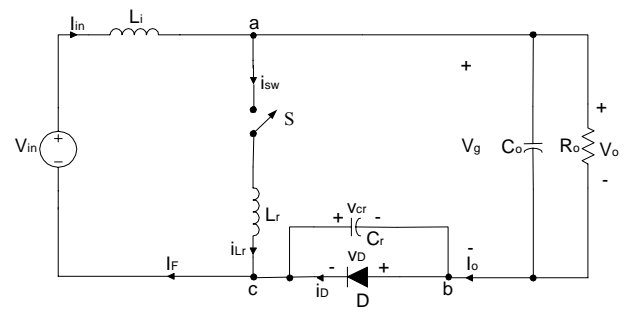

(b)

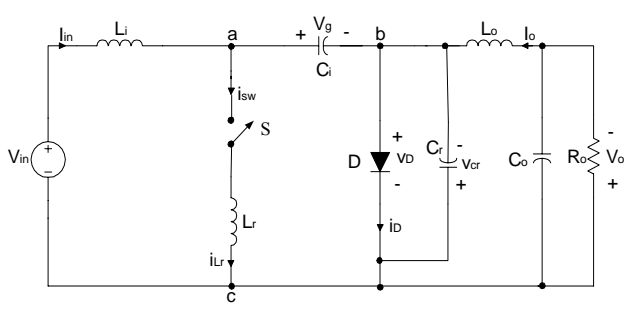

(d)

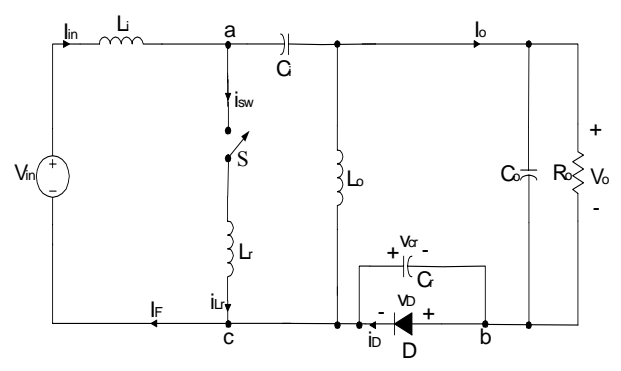

(f)

Figure 1.4: The DC-DC ZCS-QRC Family:(a) Buck. (b) Boost. (c) Buck-Boost. (d) Cuk. (e) Zeta. (f) Sepic 


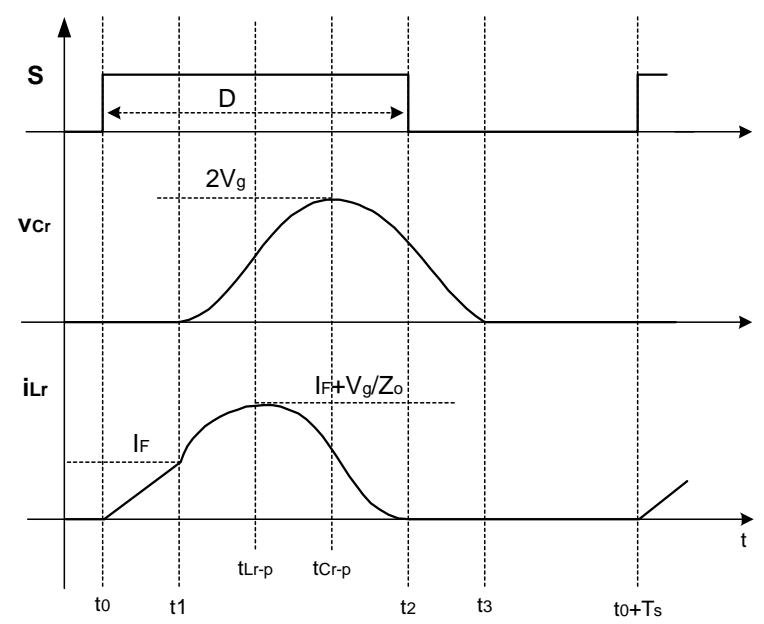

Figure 1.5: Main ZCS-QRC switching cell waveforms

Mode 3: $\left[t_{2} \leq t \leq t_{3}\right]$, starts when $S$ is turns OFF at zero. The resonant capacitor voltage starts discharging linearly until it drops to zero again causing $D$ to turn $\mathrm{ON}$ at zero voltage at $t=t_{3}$. The equivalent circuit for this mode is shown in Figure 1.6. The mathematical equation for this mode is as follow:

$$
\begin{gathered}
v_{C r}(t)=-\frac{I_{F}}{C_{r}}\left(t-t_{2}\right)+V_{g}[1-\cos \beta] \\
i_{L r}(t)=0 \\
v_{C r}\left(t_{3}\right)=0
\end{gathered}
$$


Mode 4: $\left[t_{3} \leq t \leq t_{o}+T_{s}\right]$, mode 4 is a steady-state mode and nothing happen on it until $S$ is turned ON again to start the next switching cycle. The equivalent circuit for this mode is shown in Figure 1.6. The mathematical equation for this mode is as follow:

$$
\begin{gathered}
i_{L r}(t)=0 \\
v_{C r}\left(t_{3}\right)=0
\end{gathered}
$$

\subsection{Generalized Steady-State Analysis}

From the modes of operations discussed in Section 1.5 generalized parameters can be defined and generalized equations can be derived. Section 1.6.1 defines the generalized parameters and based on them the generalized gain equation is derived in section 1.6.2. The generalized ZCS condition is discussed in 1.6.3 and finally generalized peak values for the switching cell inductor current and the switching cell capacitor voltage is discussed in section 1.6.4.

\subsubsection{Generalized Intervals Equations}

To simplify the analysis, the following time intervals are defined:

$$
\begin{gathered}
\alpha=\omega_{o}\left(t_{1}-t_{0}\right) \\
\beta=\omega_{o}\left(t_{2}-t_{1}\right) \\
\delta=\omega_{o}\left(t_{3}-t_{2}\right) \\
\eta=\omega_{o}\left(\left(t_{0}+T_{s}\right)-t_{3}\right)
\end{gathered}
$$


These intervals can be derived as follows:

- From Equations (1.2) and (1.4) :

$$
\alpha=\omega_{o}\left(t_{1}-t_{0}\right)=\frac{Z_{o} I_{F}}{V_{g}}
$$

By using the normalized parameters defined in Section 1.2:

$$
\alpha=\omega_{o}\left(t_{1}-t_{0}\right)=\frac{M I_{n F}}{Q V_{n g}}
$$

- From Equations (1.6) and (1.7) :

$$
\beta=\omega_{o}\left(t_{2}-t_{1}\right)=\sin ^{-1}\left(-\frac{M I_{n F}}{Q V_{n g}}\right)
$$

- From Equations (1.8) and (1.10) :

$$
\gamma=\omega_{o}\left(t_{3}-t_{2}\right)=\frac{Q V_{n g}}{M I_{n F}}(1-\cos \beta)
$$

- From Figure 1.5 and the above intervals :

$$
\delta=\omega_{o}\left(\left(t_{0}+T_{s}\right)-t_{3}\right)=\frac{2 \pi}{f_{n s}}-\alpha-\beta-\gamma
$$




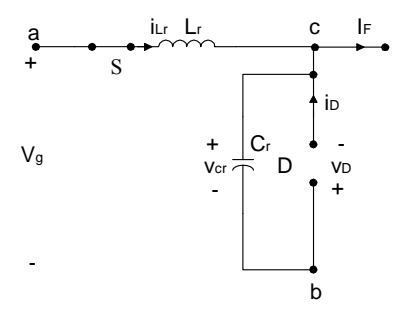

(a)

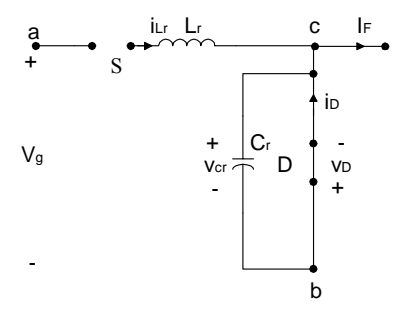

(c)

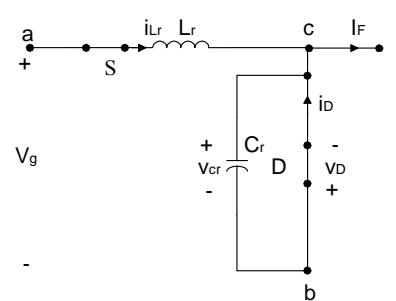

(b)

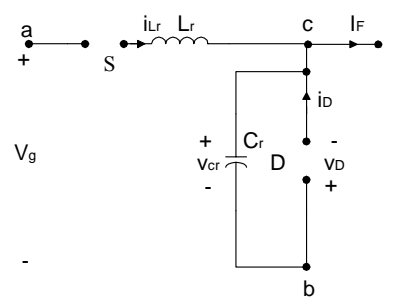

(d)

Figure 1.6: The equivalent circuits for:(a) Mode 1, (b) Mode 2,

(c) Mode 3, and (d) Mode 4

\subsubsection{Generalized Gain Equation}

The cell output to input generalized gain can be found using the cell average output voltage $V_{F}$ as follows:

$$
\begin{aligned}
V_{b c} & =V_{D}=-V_{C r} \\
& =-\frac{1}{T_{s}} \int_{t_{0}}^{t_{0}+T_{s}} V_{C r}(t) d t \\
& =-\frac{1}{T_{s}}\left[V_{g}\left(\left(t_{2}-t_{1}\right)-\frac{\sin \beta}{w_{0}}\right)-\frac{I_{F}}{2 C_{r}}\left(t_{3}-t_{2}\right)^{2}+V_{g}(1-\cos \beta)\left(t_{3}-t_{2}\right)\right]
\end{aligned}
$$


By using the normalized parameters defined in Section 1.2, we will have:

$$
V_{n b c}=\frac{f_{n s}}{2 \pi}\left[\frac{M I_{n F}}{2 Q} \gamma^{2}-V_{n g}(\beta+\gamma-\sin \beta-\gamma \cos \beta)\right]
$$

By substituting for the generalized parameters $\left(V_{n F}, V_{n g}\right.$, and $\left.I_{n F}\right)$ from Table 1.1 in Equation (1.17), we will have the gain equation for each converter in the family.

\subsubsection{Generalized ZCS Condition}

In order to achieve ZCS, which means that $S$ must be turned OFF at zero current condition. This condition can be noticed from Figure 1.5 after $t=t_{2}$ when the resonant inductor (switch) current drops to zero and before $t=t_{3}$ when the resonant capacitor voltage drops to zero causing $D$ to conduct and the resonant inductor current starts charging again. The generalized condition to achieve zero-current switching can be expresses as follows:

$$
(\alpha+\beta) \leq \frac{2 \pi}{f_{n s}}(D) \leq(\alpha+\beta+\gamma)
$$

Which limit the minimum and maximum value of the duty ratio to:

$$
\begin{gathered}
D_{\min }=\frac{f_{n s}}{2 \pi}(\alpha+\beta) \\
D_{\max }=\frac{f_{n s}}{2 \pi}(\alpha+\beta+\gamma)
\end{gathered}
$$




\subsubsection{Generalized peak resonant Inductor Current and capacitor voltage}

The peak resonant inductor current or peak switch current occurs at $t=t_{L r-p}$ when $w_{0}\left(t_{L r-p}-t_{1}\right)=\pi / 2$. By using Equation (1.6) at $t=t_{L r-p}$ :

$$
I_{n L r-P}=I_{n F}+\frac{Q V_{n g}}{M}
$$

The peak resonant capacitor voltage or peak diode voltage occurs at $t=t_{C r-p}$ when

$$
\begin{array}{r}
w_{0}\left(t_{C r-p}-t_{1}\right)=\pi \text {. By using Equation (1.5) at } t=t_{C r-p}: \\
V_{n D-p}=V_{n C r-p}=2 V_{n g}
\end{array}
$$

\subsection{Design Curves}

By substituting for the generalized parameters from Table 1.1 in the generalized equations, the design equations for each topology in the family can be found. Using MathCAD software, several design curves were plotted as shown in Figure 1.7 there are several control characteristics curves for the ZCS-QRC family, 


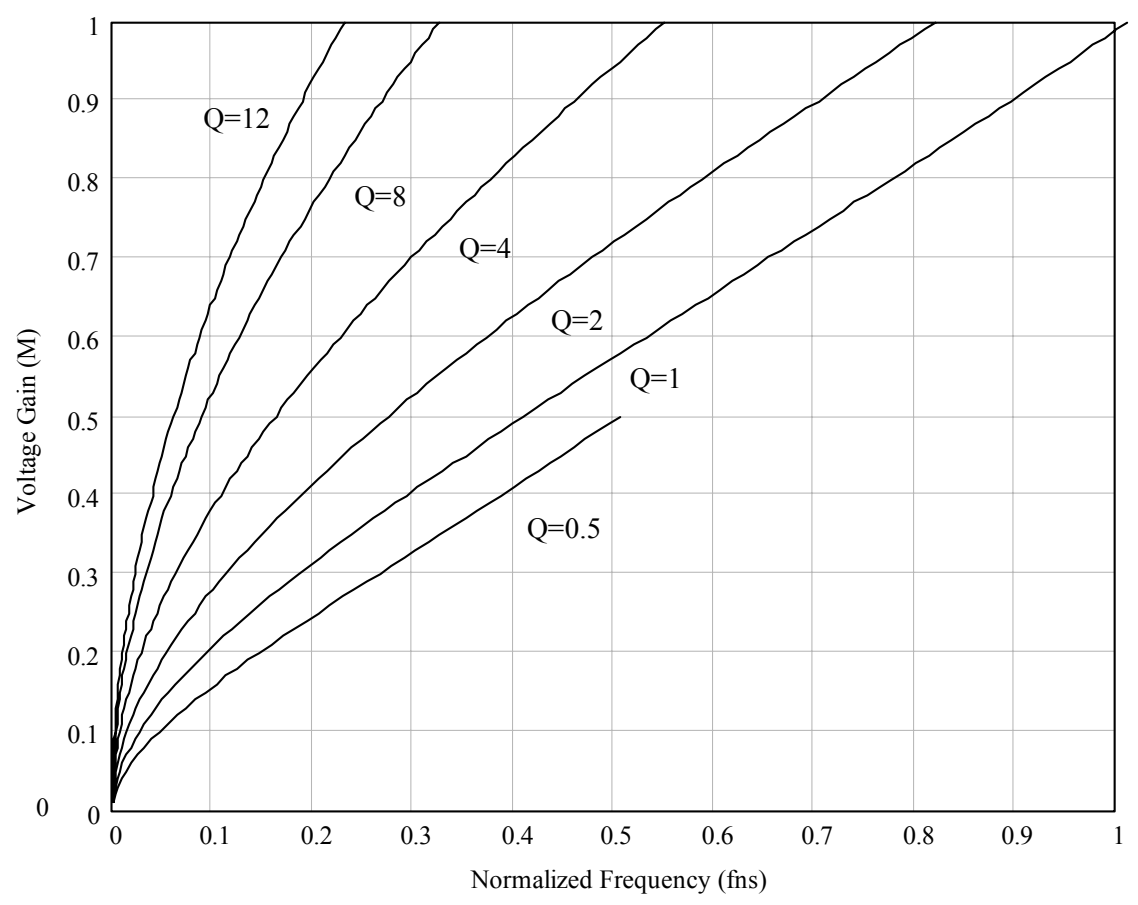

Figure 1.7a: DC voltage conversion ratio characteristics for ZCS-QRC Buck

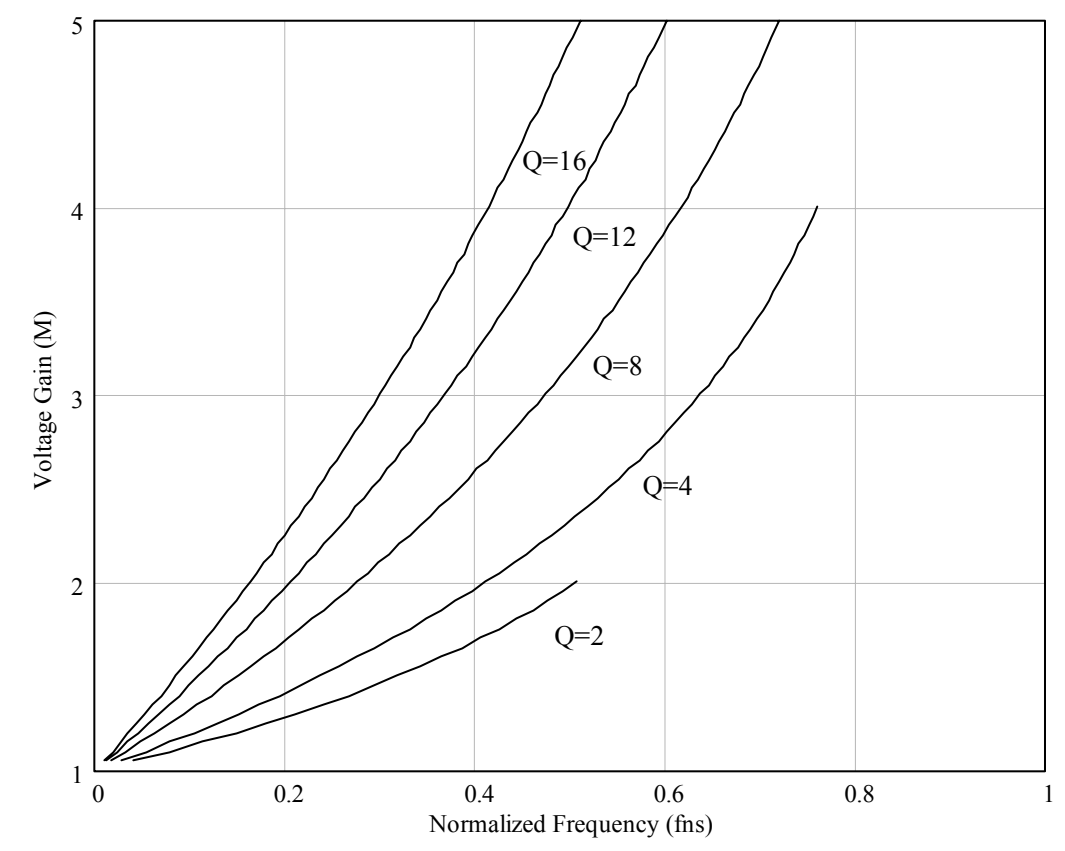

Figure 1.7b: DC voltage conversion ratio characteristics for ZCS-QRC Boost 


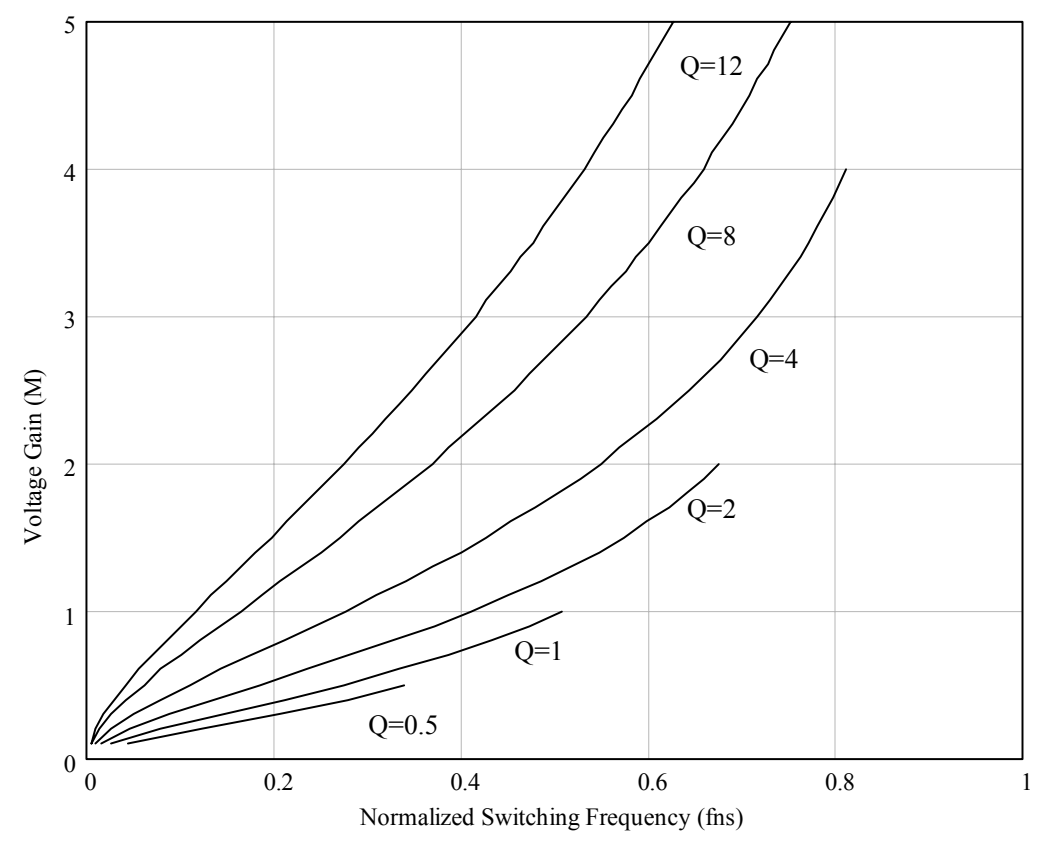

Figure 1.7c: DC voltage conversion ratio characteristics for ZCS-QRC Buck-Boost, Cuk, Zeta, and Sepic

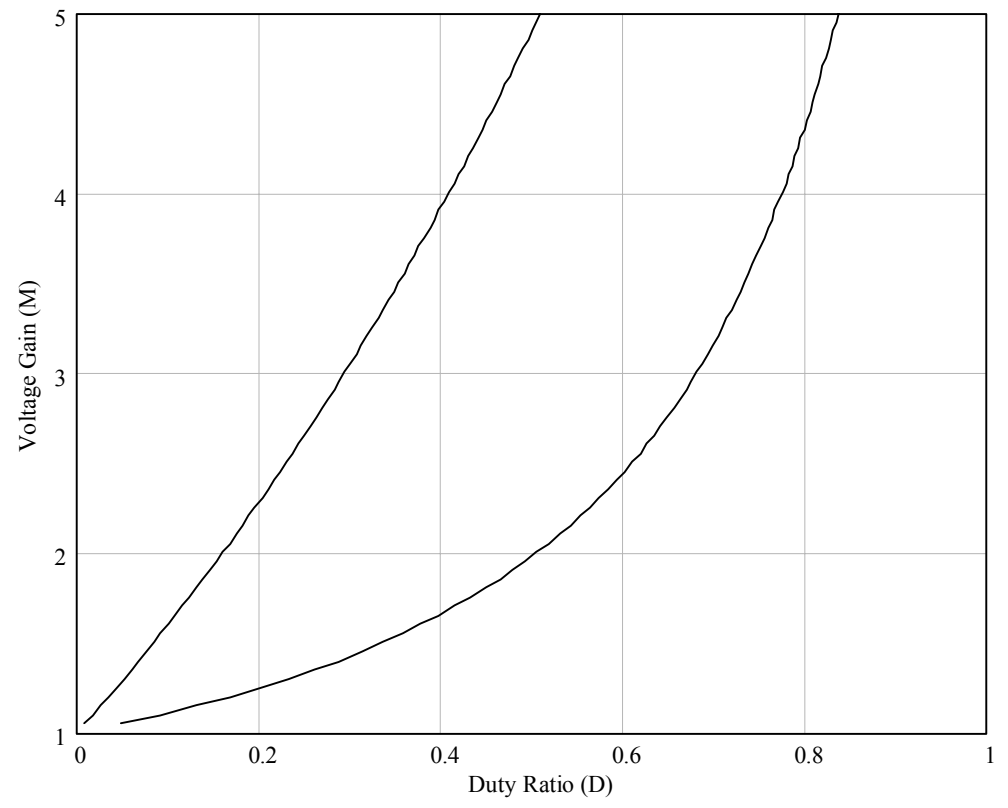

Figure 1.7d: DC voltage conversion ratio characteristics for The Minimum Allowed Duty Ratio for the ZCS-QRC Boost 


\section{CHAPTER 2}

\section{UNIFIED MODEL CONCEPT AND IMPLEMENTATION}

\subsection{Introduction}

The main idea behind the unified model is to have a unified steady-state simulation model that covers common soft-switching cells and can be used in topologies simulation. The user chooses the switching cell type and connects the simulation model to the desired topology configuration. The model provides the user with the internal switching cell information such as the switching voltage and current in addition to the regular measurement information that can be obtained from the circuitry around the unified model. To introduce the proposed unified model concept and related development issues and challenges, the zero current switching (ZCS) quasi-resonant (QRC) unified switching cell $[4,5,6,8,9]$ was selected.

Implementing the unified model in Pspice was not straightforward for the following two main reasons: First, Pspice is not a programming language, hence it doesn't have variables or basic programming elements like "For" or "While" loops, it only support very basic IF..ELSE function. Second, the final output in Pspice is the result of object interaction and hence implementing different flow charts is confusing especially when trying to control the flow of the program and the proper way of communication between the objects [17-26].

In this chapter a detailed discussion of the unified model concept and implementation is presented, next section presents the Unified Steady-State Model flow- 
chart, Section 2.3 discusses the modified flow-chart for the Zero Current Switching Quasi Resonant converter ZCS-QRC, the chapter then moves to the implementation of this flow-chart in Pspice in Section 2.4 by presenting how to build the necessary programming components for Pspice. Building functional block is Pspice should be done in certain way Section 2.5 discuss the system architecture in Pspice and finally Section 2.6 shows the detailed implementation of the system blocks in Pspice.

\subsection{Unified Steady-State Model Flow-chart}

The general flow chart of the unified model is shown in Figure 2.1. The flow chart explains the basic internal structure of the unified model and the way it works. The model starts by taking necessary inputs from the user this includes the input voltage, the switching cell inductance $L_{r}$, the switching cell capacitance $C_{r}$ and the switching frequency $f_{s}$ as a constant input to the cell or from the closed loop controller. The model then calculates the normalized switching frequency $f_{n s}$, the characteristic impedance $Z_{o}$ and the Quality factor Q. Those calculated values are constant values that will be used at a later stage in the model. Then, the model goes through several iterations, in each iteration, several sub-equations are evaluated, and the sub-equations final results are mathematically arranged and stored according to the topology, and subtracted from previously calculated $f_{n s}$ value. The result of this comparison determines if steady-state solution is reached or more iterations are still needed. After finding the steady-state solution, the final stage generates steady-state waveforms of the switching cell. 


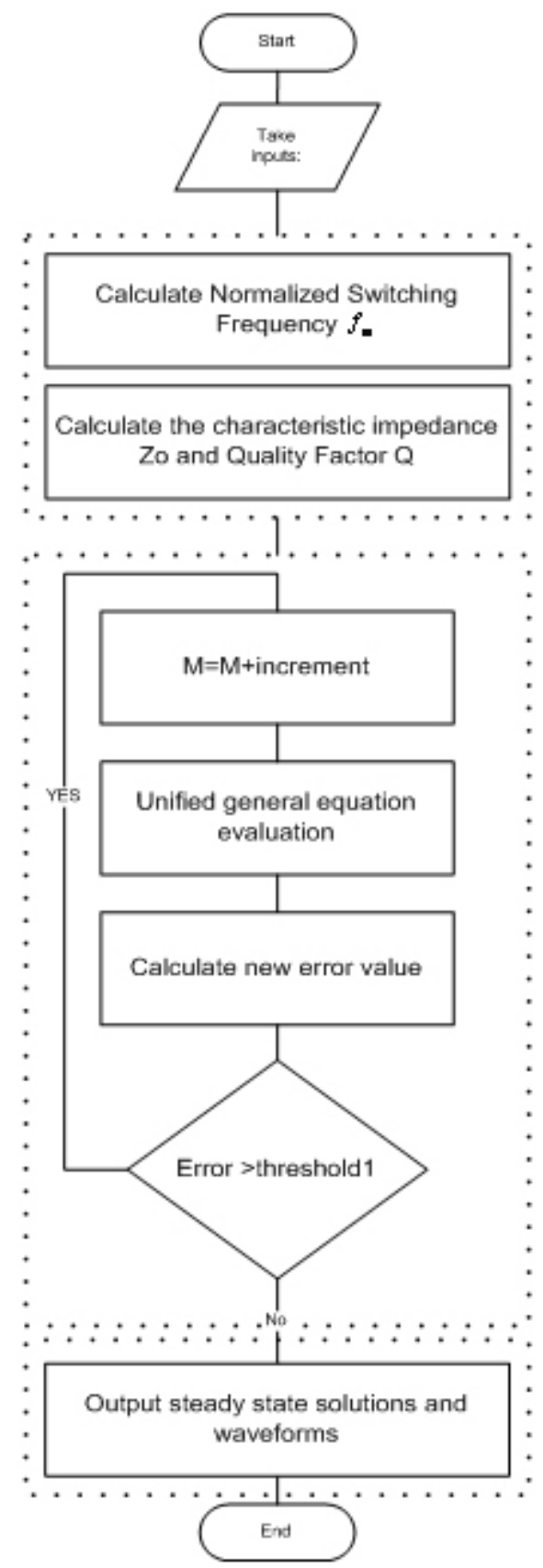

Figure 2.1: Unified Steady-State Model Flow-chart 


\subsection{ZCS-QRC Steady-State Model Flow-chart}

The derivation of the generalized gain equation for the zero current switching (ZCS) quasi-resonant (QRC) switching cell was discussed in details in the previous chapter. From Section 1.6.2:

$$
V_{n D}=\frac{f_{n s}}{2 \pi}\left[\frac{M I_{n F}}{2 Q} \gamma^{2}-V_{n g}(\beta+\gamma-\sin \beta-\gamma \cos \beta)\right]
$$

Where:

$$
\begin{gathered}
M=\frac{V_{o}}{V_{i n}} \\
f_{n s}=\frac{f_{s}}{f_{o}} \\
Q=\frac{R_{o}}{Z_{o}} \\
\alpha=\frac{M I_{n F}}{Q V_{n g}} \\
\beta=\sin ^{-1}\left(-\frac{M I_{n F}}{Q V_{n g}}\right) \\
\gamma=\frac{Q V_{n g}}{M I_{n F}}(1-\cos \beta) \\
\delta=\frac{2 \pi}{f_{n s}}-\alpha-\beta-\gamma
\end{gathered}
$$

And the generalized transformation table from Section 1.3 is shown in table 2.1. 
Table 2.1: Generalized Transformation Table

\begin{tabular}{|c|c|c|c|}
\hline $\begin{array}{c}\text { Normalized } \\
\text { Parameters }\end{array}$ & Buck & Boost & Buck -Boost \\
\hline$V_{n g} I_{n F}$ & 1 & $\mathrm{M}$ & $1+\mathrm{M}$ \\
\hline$V_{n F}, I_{n b}, I_{n T}$ & $1-\mathrm{M}$ & 1 & 1 \\
\hline$V_{n b c}$ & $-\mathrm{M}$ & $1-\mathrm{M}$ & $-\mathrm{M}$ \\
\hline
\end{tabular}

Using the ZCS-QRC generalized gain equation 2.1 and the generalized transformation table 2.1 the generalized gain equation for ZCS-QRC buck converter becomes:

$$
-M=\frac{f_{n s}}{2 \pi}\left[\frac{M}{2 Q} \gamma^{2}-(\beta+\gamma-\sin \beta-\gamma \cos \beta)\right]
$$

Where:

$$
\begin{gathered}
\alpha=\frac{M}{Q} \\
\beta=\sin ^{-1}\left(-\frac{M}{Q}\right) \\
\gamma=\frac{Q}{M}(1-\cos \beta)
\end{gathered}
$$

Equation 2.9 is a non-linear equation with the steady-state gain solution $M$ in both sides of the equation. To simplify the solving algorithm the equation was rearranged and solved in terms of $f_{n s}$ as shown in equation 2.13.

$$
f_{n s}=\frac{-2 \pi M}{\frac{M}{2 Q} \gamma^{2}-(\beta+\gamma-\sin \beta-\gamma \cos \beta)}
$$


Using equation 2.13, the normalized switching frequency value $f_{n s}$ is known, and the steady-state gain value $M$ is not, one solution to find steady-state gain value would be increasing $M$ value iteratively in small steps, in each step a new value of $f_{n s}$ is solved by using equation 2.13 and the result is subtracted from the true $f_{n s}$ value calculated using equation 2.3 to get an error signal. The process keeps going till the error goes below predetermined threshold level. Figure 2.2 shows the solution flowchart.

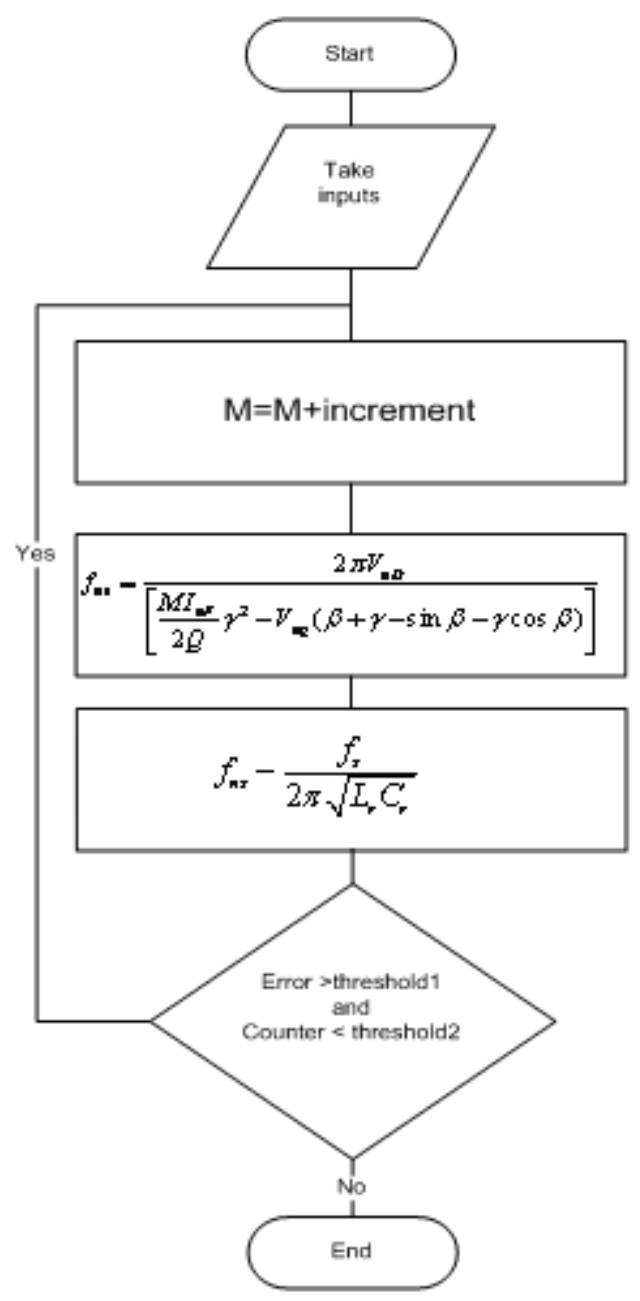

Figure 2.2: ZCS-QRC buck unified equation solution Flow-chart 
To prove the validity of the algorithm, the solution flow-chart was programmed in MATLAB and tested using soft-switching cell inductance $L_{r}=3 \mu \mathrm{H}$, soft-switching cell capacitance $C_{r}=0.02 \mu \mathrm{F}$, switching frequency $f_{s}=250 \mathrm{KHz}$, input voltage $V_{\text {in }}=25 \mathrm{~V}$ and load $R_{o}=12 \Omega$. The program proved the correctness of the approach and converges to a solution if there is any. Figure 2.3 shows the steady-state waveforms for the switching cell inductor current $I_{L r}$ and switching cell capacitor voltage $V_{C r}$.
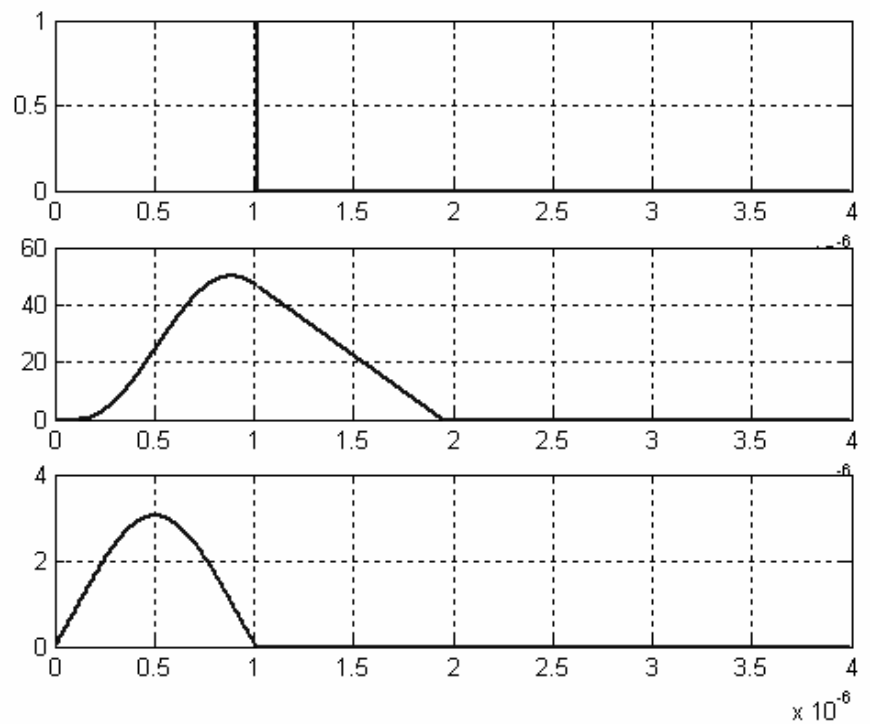

Figure 2.3: ZCS-QRC buck steady-state waveforms using MATLAB

The next part of this chapter is the implementation of the above flowchart in the Pspice. As it was discussed in section 2.1, Pspice implementation requires creating different programming components, and building function in certain hierarchical structure. The next section discusses those issues, and the actual implementation in Pspice [24-27]. 


\subsection{Building programming components}

To overcome the lack of programming elements in Pspice, necessary programming tools were developed based on existing Pspice elements. The two most important elements considered are creating variables and creating loops. Section 2.4.1 presents the implementation of variables in Pspice and section 2.4 .2 discusses the implementation of FOR and WHILE loop in Pspice.

\subsubsection{Creating variables in Pspice}

Variables are symbols denoting a quantity; Pspice supports a basic type of variable called PARAM that once defined can not be changed, as shown in Figure 2.4. This kind of data type that once defined can not be changed is called Constant in other programming languages. PARAM can not be used in dynamic programs where the state of a variable needs to be updated continuously, so there is a need to create objects in Pspice that can store and update its state $[17,18,19]$.

\begin{tabular}{l} 
PARAMETERS: \\
\hline L_p $=100 \mathrm{uH}$ \\
$n=15$ \\
$\mathrm{C}=220 \mathrm{uF}$ \\
$\mathrm{R}=1.0 \mathrm{Kohm}$
\end{tabular}

Figure 2.4: Constant variables in Pspice

Dynamic objects or variables can be created in Pspice by using a capacitor with an additional circuit to control and synchronize the time of charging and discharging of the capacitor to indicate variable change. Figure 2.5 shows the proposed circuit. 


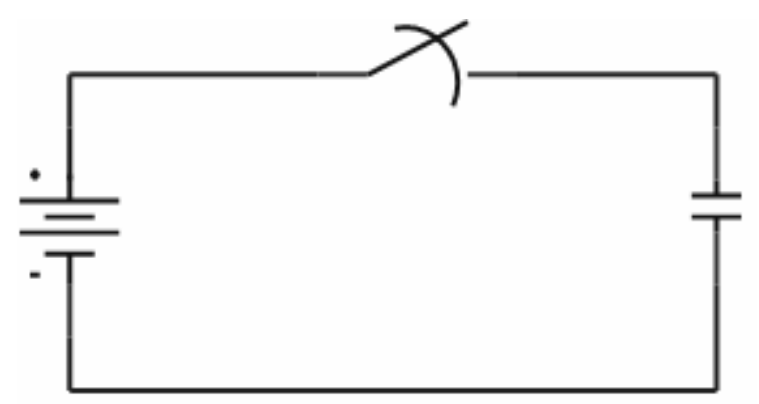

Figure 2.5: Creating variables in Pspice

The circuit works as follows: Turn the switch ON and charge the capacitor to the desired value, then turn the switch OFF. Turning the switch ON and OFF depends on a logic circuit; the complexity of this logic circuit varies according to the complexity of the application, but in its simplest form it is a comparator that compares the capacitor voltage to a certain reference. When the capacitor charges to the reference value, the switch turns OFF.

If the capacitor voltage or variable value is at certain value $\mathrm{x}$ and the value needs to be updated to a lower value, the same logic is applied, but the capacitor is now discharged using another switch and source of opposite polarity.

\subsubsection{Creating Loops in Pspice}

Loops are control structures that allow instructions to be executed repeatedly based on a given Boolean condition. There are several forms of Loops in different programming languages, most famous are FOR and WHILE loops. FOR loop starts from 
an initial value and terminates at a final value with specified increment between the start and the end. In WHILE loop the code keeps executing repeatedly until a specific Boolean condition is met.

\subsubsection{Creating FOR Loop}

FOR loop was built in Pspice using a ramp source which has a start value, an end value, a capacitor to store the value and finally an "ABM" Pspice block which contains the condition to break out from the loop. Figure 2.6 shows the basic FOR loop circuit.

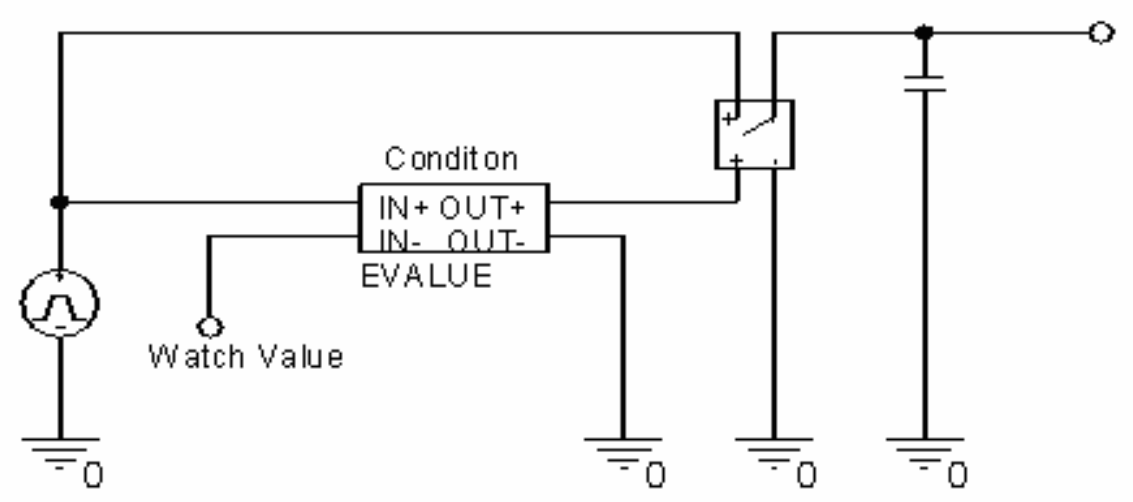

Figure 2.6: FOR loop circuit in Pspice

The circuit works as follows; The ramp source sets the FOR loop start value and end value, and the ABM block keeps monitoring the ramp source value and compares it to a "watch value" used to break out from the loop. As long as the programmed condition in the ABM block is true, the ABM block outputs a logic 1 to the "Sbreak" switch, hence the switch is $\mathrm{ON}$ and the capacitor is charging and storing the variable value. When the programmed condition becomes false, the ABM block outputs logic 0 to the "Sbreak" switch, hence the switch now is OFF and the capacitor stops charging and keeps the last 
charged value. The capacitor keeps different values since it is an RC circuit with resistance $\mathrm{R}$ equal to infinity. So the discharge time equals infinity.

\subsubsection{Creating WHILE Loop}

WHILE loop was built in Pspice using the same idea as FOR loop, but instead of using a ramp source with start value and an end value as a source for incrementing the loop, an ABM block with special variable called TIME was used. TIME is a Pspice variable that contains information about the simulation time. It starts at the beginning of the simulation with zero value and keeps incrementing as long as the simulation is running. Figure 2.7 shows the basic WHILE loop circuit.

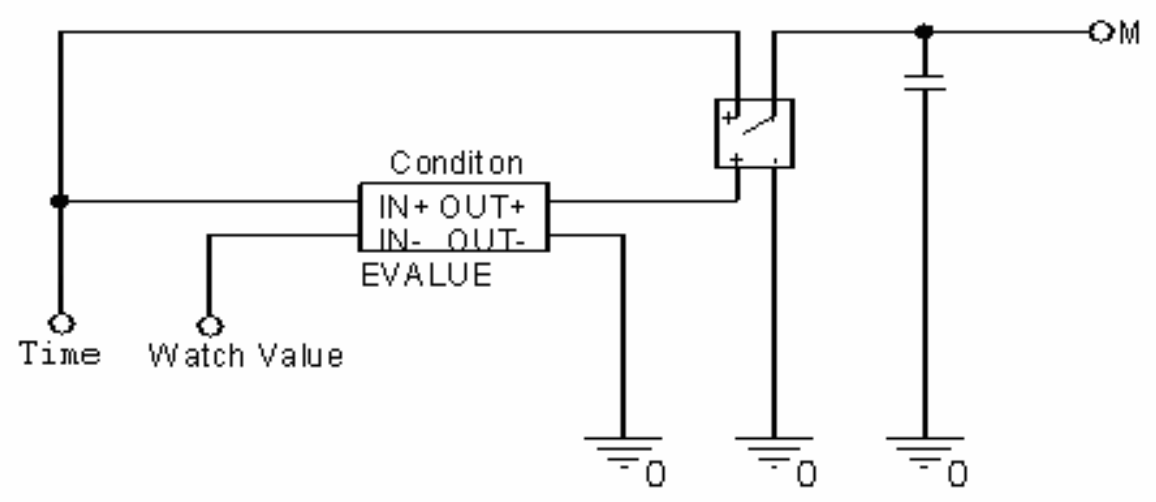

Figure 2.7: WHILE loop circuit in Pspice

The circuit works as follows: The TIME block starts at zero and keeps incrementing until the end of the simulation time. The ABM block keeps monitoring the TIME source value and compares it to a "watch value" used to break out from the loop. As long as the programmed condition in the ABM block is true, the ABM block outputs a 
logic 1 to the "Sbreak" switch, hence the switch is ON and the capacitor is charging and storing the variable value. When the programmed condition becomes false, the ABM block outputs logic 0 to the "Sbreak" switch, hence the switch is OFF and the capacitor stops charging and keeps the last charged value.

\subsection{Developing System Architecture}

Pspice has a different architecture than conventional programming languages. In conventional programming languages, the programmer writes a set of lines that compose certain functionality. The execution of the program or the function starts from the first line and ends by the last line, so the flow of the program and the direction of execution are determined by the order of the written lines. On the other hand, Pspice is an object-based program; the user places several objects in the work space, connects them together and the final outcome is the result of the interaction of those objects. So implementing different flowcharts in Pspice requires more effort, especially when trying to control the flow of the program and the proper communication between objects.

The unified equation was programmed in Pspice using special architecture that divides the programs into several functions and implements those functions using Pspice blocks that interact with each other. The unified general equation architecture includes a main block that passes certain values to other "sub-blocks." Those sub-blocks then process the information and update the Main-Block with the new values. Based on those new values, the Main-Block decides whether to continue solving or to stop when a steady-state solution is reached. Figure 2.8 shows the system architecture idea. 


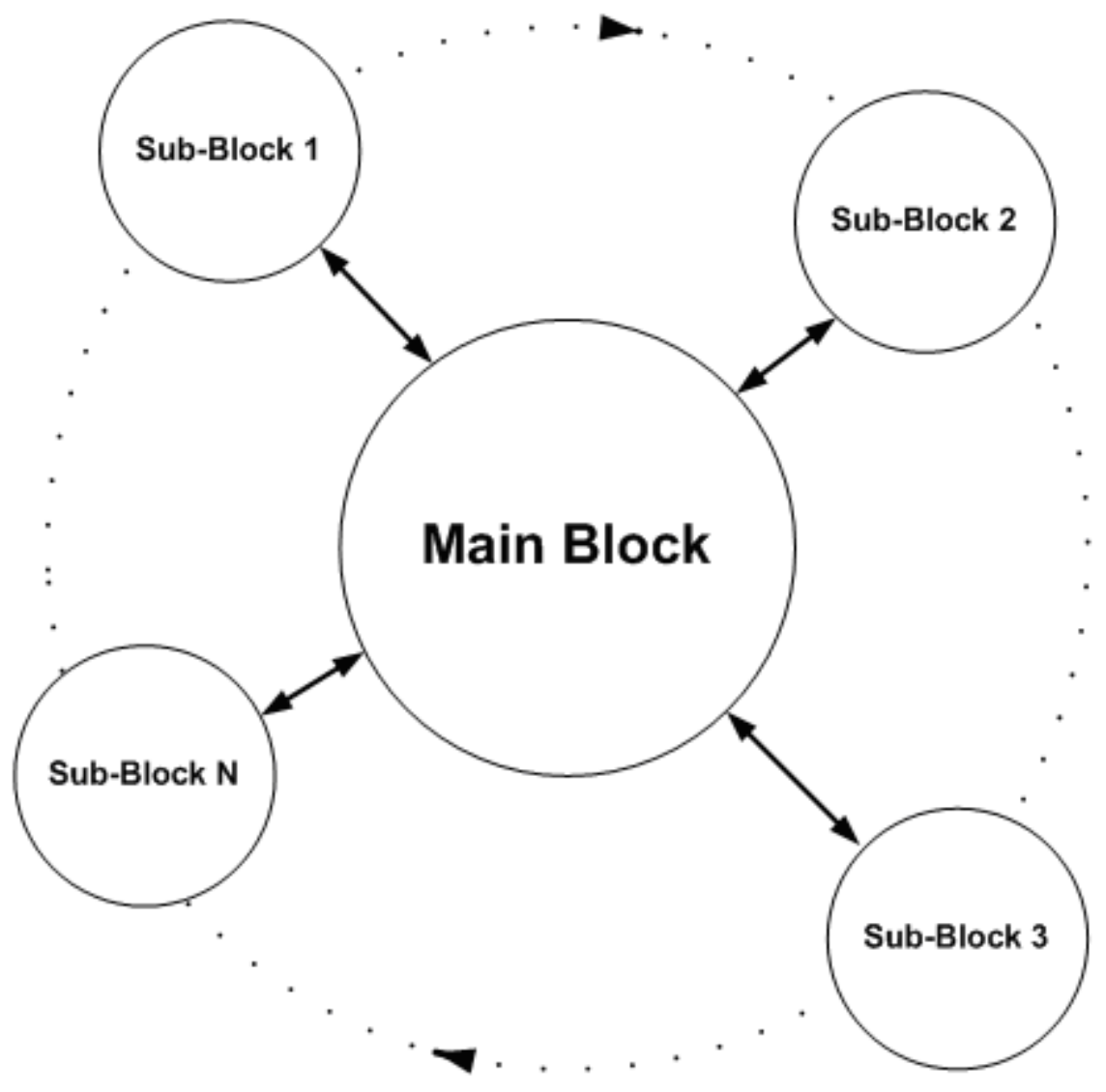

Figure 2.8: System Architecture idea

Architecture implementation in Pspice is shown in Figure 2.9, where $F_{n s}$ Block is the block responsible for calculating the normalized switching frequency, $Q Z$ Block is the block responsible for calculating the characteristic impedance $Z_{o}$ and the Quality factor $Q . B G$ Block is the block responsible for calculating Alpha, Beta and Gamma values, ERR Block is the block responsible for calculating the error between the calculated $F_{n s}$ value (calculated from M, Gamma, Beta. Q) and the true $F_{n s}$ value (Calculated from user inputs of $L_{r}, C_{r}$ and $F_{s}$ ). SOL Block is the block responsible for 
generating the steady-state gain value $M$ and, finally, the $D R W$ Block is the block responsible for generating the steady-state waveforms for the switching cell inductor current $\mathrm{I}_{\mathrm{Lr}}$ and the switching cell capacitor voltage $\mathrm{V}_{\text {cr.. }}$
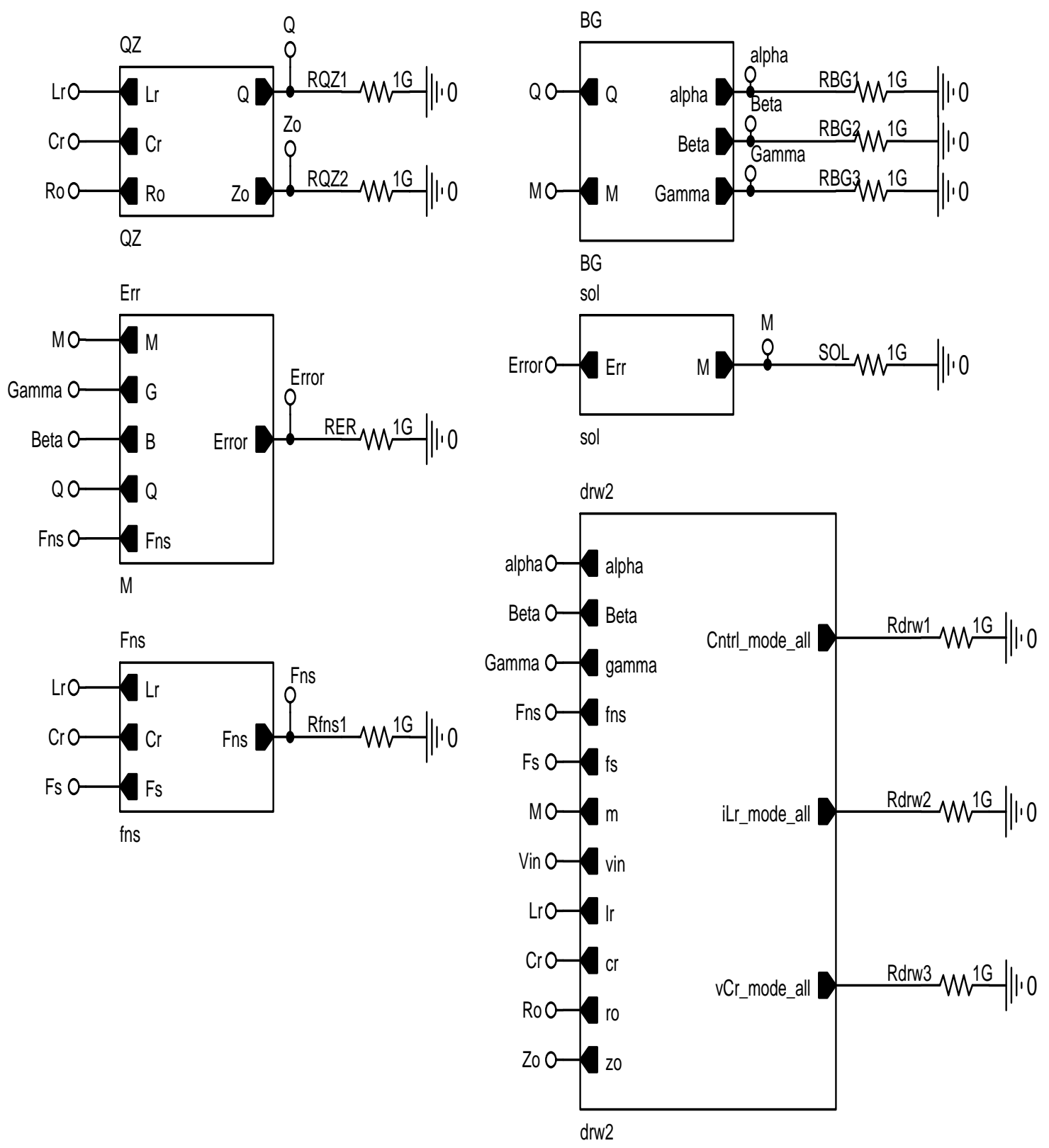

Figure 2.9: System Architecture Implementation in Pspice 


\subsection{Developing System Sub-blocks}

The development procedure of the system sub-blocks is discussed in this section; the discussion includes the main function of each block along with its implementation details.

\subsubsection{Unified model Inputs}

The Unified model uses five variables to do all the necessary calculations. The required inputs from the user include the soft-switching cell inductance $L_{r}$, the softswitching cell Capacitance $C_{r}$, the topology output resistance $R_{o}$, the required switching frequency $f_{s}$ and the topology input voltage $V_{i n}$. Figure 2.10 shows the required system inputs.

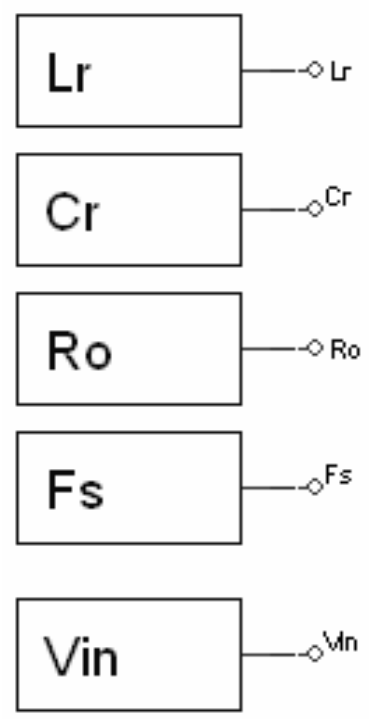

Figure 2.10: Required System Inputs 


\subsubsection{Normalized Switching Frequency Block}

The normalized switching frequency, or $f_{n s}$, Block is the block responsible for calculating the normalized switching frequency for the unified model. Figure 2.11 shows the $f_{n s}$ block.

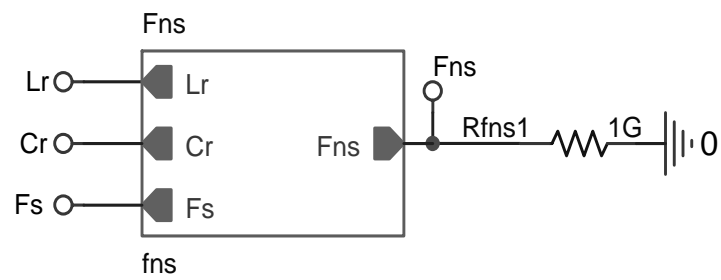

Figure 2.11: Normalized Switching Frequency Block

The normalized switching frequency $f_{n s}$ is the ratio of switching frequency $f_{s}$ to the resonant angular frequency $f_{o}$.

$$
f_{n s}=\frac{f_{s}}{f_{o}}
$$

The resonant angular frequency $w_{o}$ is given by the relation:

$$
w_{o}=\frac{1}{\sqrt{L_{r} C_{r}}}
$$

Or

$$
f_{o}=\frac{1}{2 \pi \sqrt{L_{r} C_{r}}}
$$

Substituting Equation 2.16 in Equation 2.14: 


$$
f_{n s}=2 \pi \cdot f_{s} \cdot \sqrt{L_{r} C_{r}}
$$

To implement this equation and any other equation in Pspice, a new technique called "Block Concatenation" is followed. Block-Concatenation is a procedure to implement mathematical functions in Pspice. The method starts by breaking down the equation into its simplest form, then the equation input and output are identified. The next step involves dividing the equation into a group of sub-functions with certain logical or mathematical relations between them, and finally those sub-functions are arranged in a certain hierarchical structure to achieve the desired final function or equation. The BlockConcatenation development procedure for Normalized Switching Frequency $f_{n s}$ block is shown in table 2.2 .

Table 2.2: development procedure for $f_{n s}$ block

\begin{tabular}{|c|l|c|}
\hline Procedure & & \\
\hline 1 & Equation Inputs & $f_{s}, L_{r}, C_{r}$ \\
\cline { 2 - 3 } & Equation Output & $f_{n s}$ \\
\hline 2 & $\begin{array}{l}\text { Mathematical and } \\
\text { Logical Expressions }\end{array}$ & $\begin{array}{c}\text { Multiplication and } \\
\text { Division }\end{array}$ \\
\hline 3 & Required sub-blocks & $\begin{array}{c}\text { Two blocks are needed: } \\
\text { one to calculate } \sqrt{L_{r} C_{r}} \\
\text { and the other to } \\
\text { perform division. }\end{array}$ \\
\hline
\end{tabular}

Based on this procedure, a flowchart was developed to implement the $f_{n s}$ block. Figure 2.12 shows the flowchart. The flowchart was converted at a later stage into functional 
Pspice block. Figure 2.13 shows Pspice implementation of the Normalized Switching Frequency, $f_{n s}$ block.

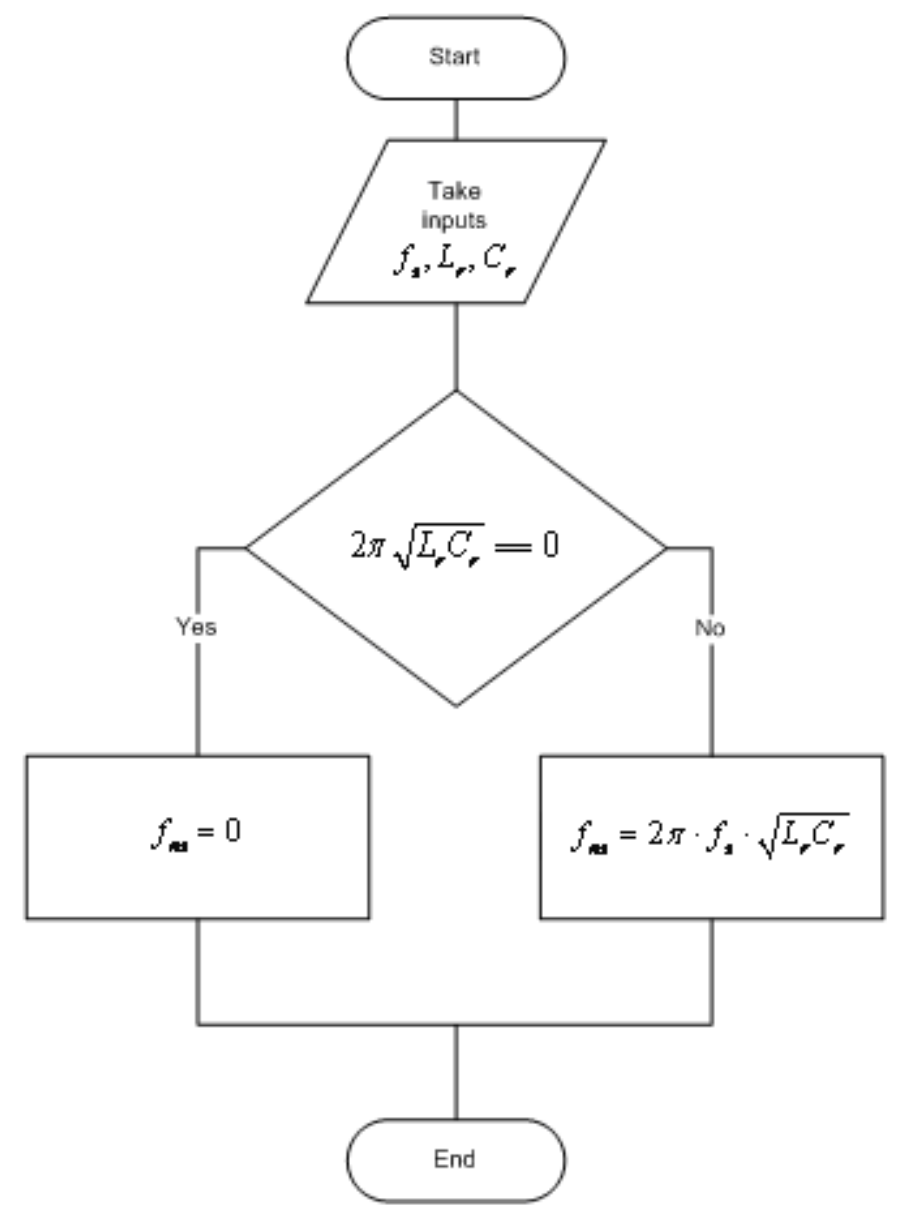

Figure 2.12: Normalized Switching Frequency Block flowchart

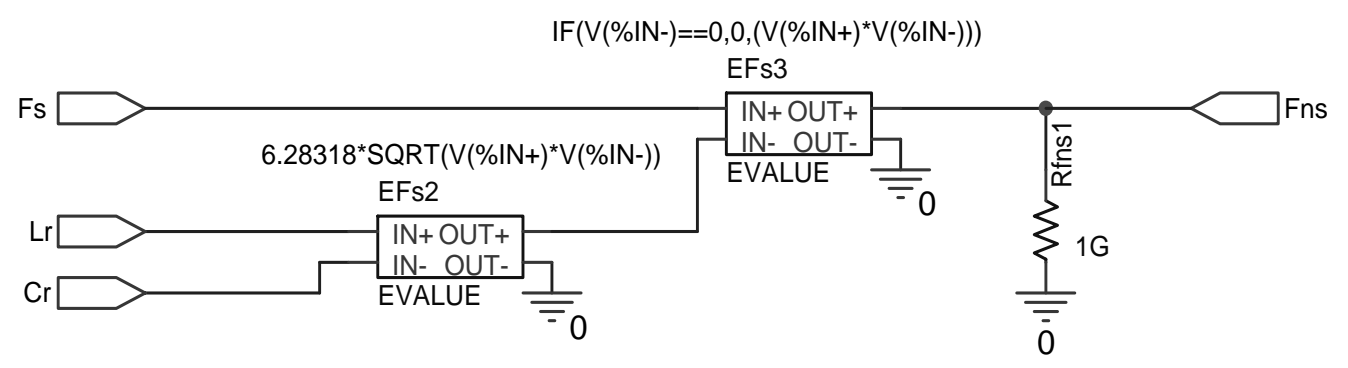

Figure 2.13: Pspice implementation of $f_{n s}$ Block 
The block was tested using soft-switching cell inductance $L_{r}=3 \mu \mathrm{H}$, softswitching cell capacitance $C_{r}=0.02 \mu \mathrm{F}$, switching frequency $f_{s}$ that steps from $500 \mathrm{KHz}$ to $250 \mathrm{KHz}$. After substituting those values in Equation 2.17 the expected theoretical results are:

$$
\begin{aligned}
& f_{n s 1}=2 \pi \cdot f_{s} \cdot \sqrt{L_{r} C_{r}}=2 \pi \cdot 500 \times 10^{3} \cdot \sqrt{\left(3 \times 10^{-6}\right) \cdot\left(0.02 \times 10^{-6}\right)}=0.769 \\
& f_{n s 2}=2 \pi \cdot f_{s} \cdot \sqrt{L_{r} C_{r}}=2 \pi \cdot 250 \times 10^{3} \cdot \sqrt{\left(3 \times 10^{-6}\right) \cdot\left(0.02 \times 10^{-6}\right)}=0.385
\end{aligned}
$$

Figure 2.14 shows the simulation results. From Figure 2.14, the simulation results match with the theoretical results.

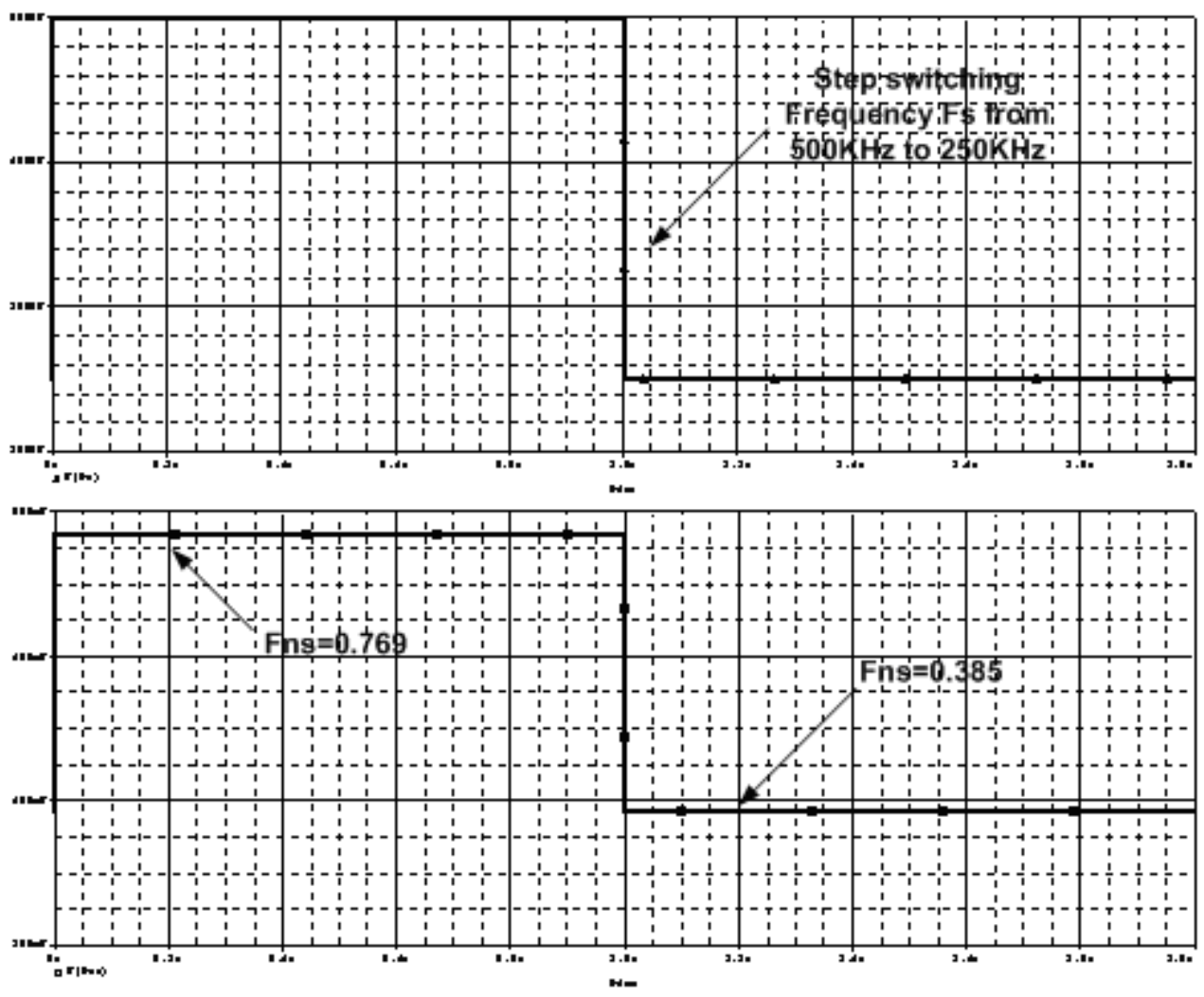

Figure 2.14: Pspice Simulation results for $f_{n s}$ Block 


\subsubsection{Quality Factor \& Characteristics Impedance Block “QZ Block”}

The Quality Factor \& Characteristics Impedance Block or QZ Block, is the block responsible for calculating the Characteristics Impedance $Z_{o}$ and the Quality Factor $Q$ for the unified model. As seen from equation 2.13 the Characteristics Impedance and the Quality Factor are important quantities used for other sub-blocks calculations. Figure 2.15 shows the $Q Z$ block.

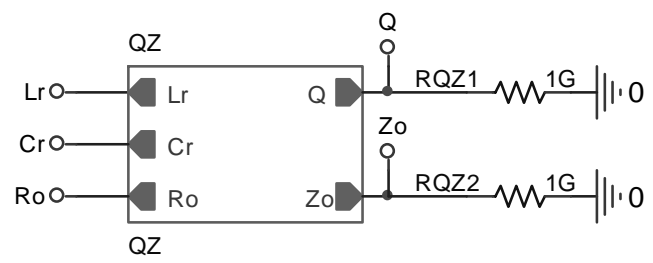

Figure 2.15: Quality Factor \& Characteristics Impedance Block

The Quality Factor $Q$ and the Characteristics Impedance $Z_{o}$ are mathematically related and can be calculated from each other, so they were placed in the same block. The characteristic impedance $Z_{o}$ can be calculated from Equation 2.18 and the Quality Factor $Q$ can be calculated from Equation 2.19. The relation between $Z_{o}$ and $Q$ is shown in Equation 2.20.

$$
\begin{gathered}
Z_{o}=\sqrt{\frac{L_{r}}{C_{r}}} \\
Q=\frac{R_{o}}{Z_{o}}
\end{gathered}
$$




$$
Q=\frac{R_{o}}{\sqrt{\frac{L_{r}}{C_{r}}}}
$$

Using the Block-Concatenation technique, the development procedure for the $Q Z$ block is shown in Table 2.3

Table 2.3: development procedure for $Q Z$ block

\begin{tabular}{|c|l|c|}
\hline Procedure & & \\
\hline 1 & Equation Inputs & $R_{o}, L_{r}, C_{r}$ \\
\cline { 2 - 3 } & Equation Output & $Z_{o}$ and $Q$ \\
\hline 2 & $\begin{array}{l}\text { Mathematical and } \\
\text { Logical Expressions }\end{array}$ & $\begin{array}{c}\text { Multiplication and } \\
\text { Division }\end{array}$ \\
\hline 3 & Required sub-blocks & $\begin{array}{c}\text { Two blocks are needed: } \\
\text { one to calculate } \\
\sqrt{L_{r} / C_{r}} \text { and the other } \\
\text { to perform division. }\end{array}$ \\
\hline
\end{tabular}

Based on this procedure, a flowchart was developed to implement the QZ block.

Figure 2.16 shows the flowchart. The flowchart was converted at a later stage into functional Pspice block. Figure 2.17 shows Pspice implementation of the Quality Factor and the Characteristics Impedance QZ block. 


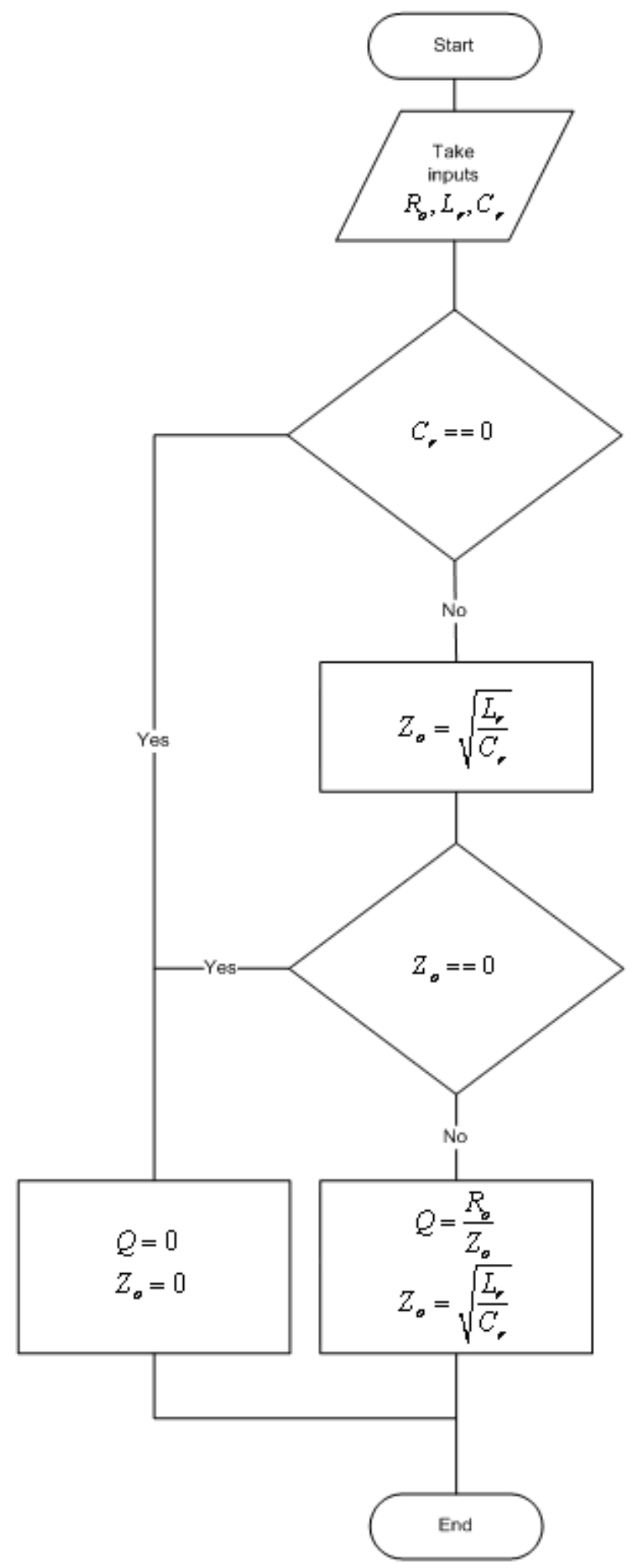

Figure 2.16: Quality Factor and the Characteristics Impedance QZ block flowchart 


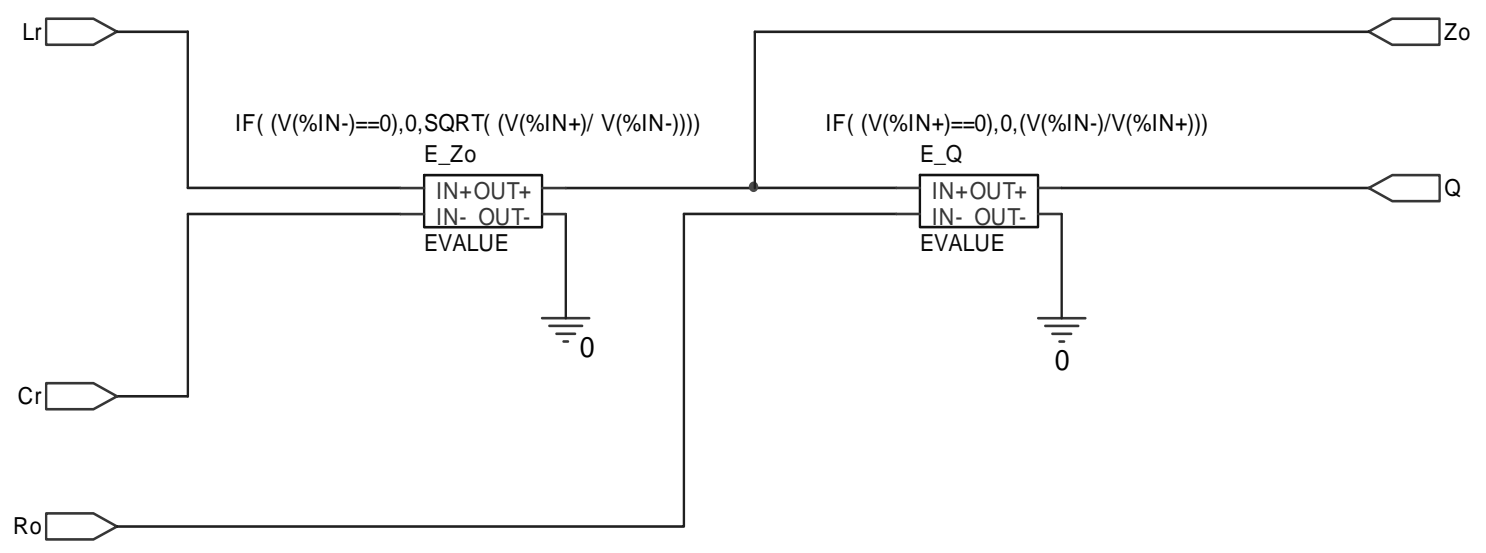

Figure 2.17: Pspice implementation of QZ Block

The QZ block was tested using soft-switching cell inductance $L_{r}=3 \mu \mathrm{H}$ and soft-switching cell capacitance $C_{r}=0.02 \mu \mathrm{F}$. Applying equations 2.18 and 2.19 respectively, the expected theoretical results are shown below and Figure 2.18 shows the simulation results. From Figure 2.18 the simulation results matches with the calculated theoretical values.

$$
\begin{aligned}
& Z_{o}=\sqrt{\frac{L_{r}}{C_{r}}}=\sqrt{\frac{3.0 \times 10^{-6}}{0.02 \times 10^{-6}}}=12.2474 \\
& Q=\frac{R_{o}}{Z_{o}}=\frac{12}{12.2474}=0.97979
\end{aligned}
$$




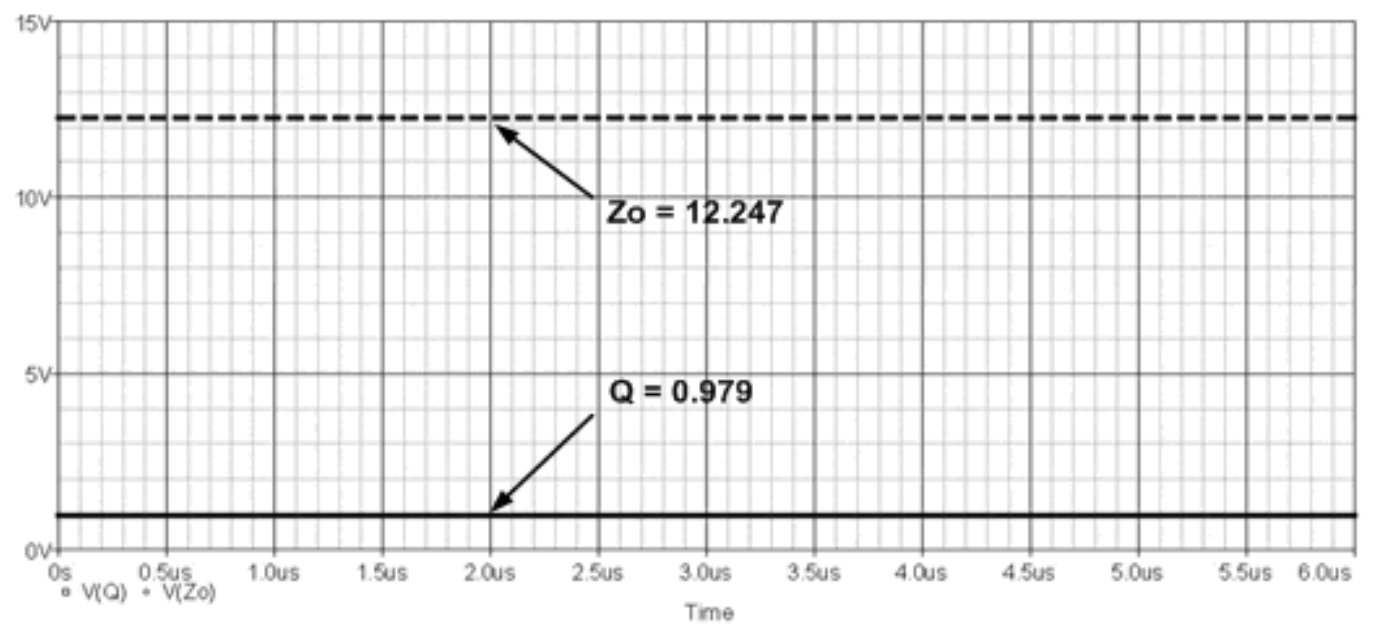

Figure 2.18: Pspice Simulation results for $f_{n s}$ Block

\subsubsection{Beta, Gamma and Alpha Block "BG Block"}

The Beta, Gamma and Alpha, or the BG Block, is the block responsible for calculating the values of Alpha " $\alpha$ ", Beta " $\beta$ " and Gamma " $\gamma$ " for the unified model. Alpha, Beta and Gamma are the generalized interval equations used to simplify the generalized analysis and are defined in Equations 1.13, 1.14 and 1.15 respectively. As can be seen from Equation 2.13, Alpha, Beta and Gamma are important quantities used for later calculations. Figure 2.19 shows the $B G$ block.

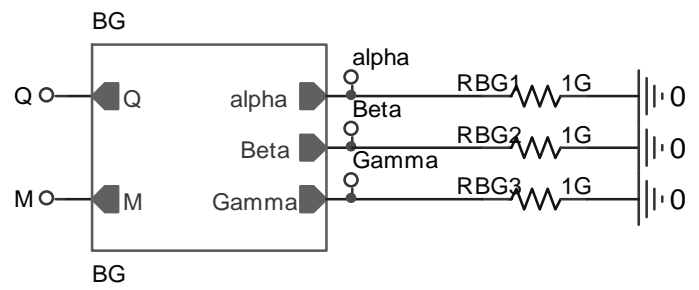

Figure 2.19: Alpha, Beta and Gamma Block 
From Figure 2.19 the $B G$ block takes two inputs: the Quality factor $Q$ and the converter output to input voltage gain $M$. From $Q$ and $M$ the $B G$ block calculates the corresponding values of Alpha, Beta and Gamma. The $B G$ block differs from the previous two blocks in two main ways: first, it depends on other block calculations to generate its output. Second, it has a two-way communication with the Main block - The Main block is the "SOL" block defined in Section 2.6.6 -- in other words, the BG block takes the voltage gain $M$ from the main block and for every iteration, it updates the Main block with new values of Alpha, Beta and Gamma.

Using Alpha, Beta and Gamma generalized interval equations defined in Equations 1.13, 1.14 and 1.15, and applying the Generalized Transformation Table defined in Table 2.1, the generalized interval equations for the buck converter were obtained and are defined in Equations 2.10, 2.11 and 2.12. Those equations are repeated here as a reference for the $B G$ block flowchart.

$$
\begin{gathered}
\alpha=\frac{M}{Q} \\
\beta=\sin ^{-1}\left(-\frac{M}{Q}\right)=\sin ^{-1}(-\alpha) \\
\gamma=\frac{Q}{M}(1-\cos \beta)
\end{gathered}
$$

Implementing the Block-Concatenation technique, the development procedure for the $B G$ block is shown in Table 2.4. Based on this procedure, a flowchart was developed to implement the $B G$ block. Figure 2.20 shows the flowchart. 
Table 2.4: development procedure for $B G$ block

\begin{tabular}{|c|l|c|}
\hline Procedure & & \\
\hline 1 & Equation Inputs & $\alpha, M$ \\
\cline { 2 - 3 } & Equation Output & $\alpha, \gamma$ \\
\hline 2 & $\begin{array}{l}\text { Mathematical and } \\
\text { Logical Expressions }\end{array}$ & $\begin{array}{c}\text { Multiplication and } \\
\text { Division }\end{array}$ \\
\hline 3 & Required Sub-Blocks & $\begin{array}{c}\text { Three blocks are } \\
\text { needed: one to } \\
\text { calculate } \alpha \text { and the } \\
\text { other two for } \beta, \gamma .\end{array}$ \\
\hline
\end{tabular}

As shown in Equation 2.11 the Beta block uses an inverse sine function to calculate the $\beta$ value. Pspice has a $S I N^{-1}$ function that can be programmed within Analog Behavior Model $A B M$ to implement the Beta block. During the simulation, the Beta block kept running into convergence problems. After debugging the source of error, it was found that the input to the $S I N^{-1}$ block was sometimes greater than one, which means the result will be imaginary. Although Pspice documentation states that Pspice is capable of handling imaginary numbers, the simulation proved the opposite. This imaginary value causes the block to fail. 


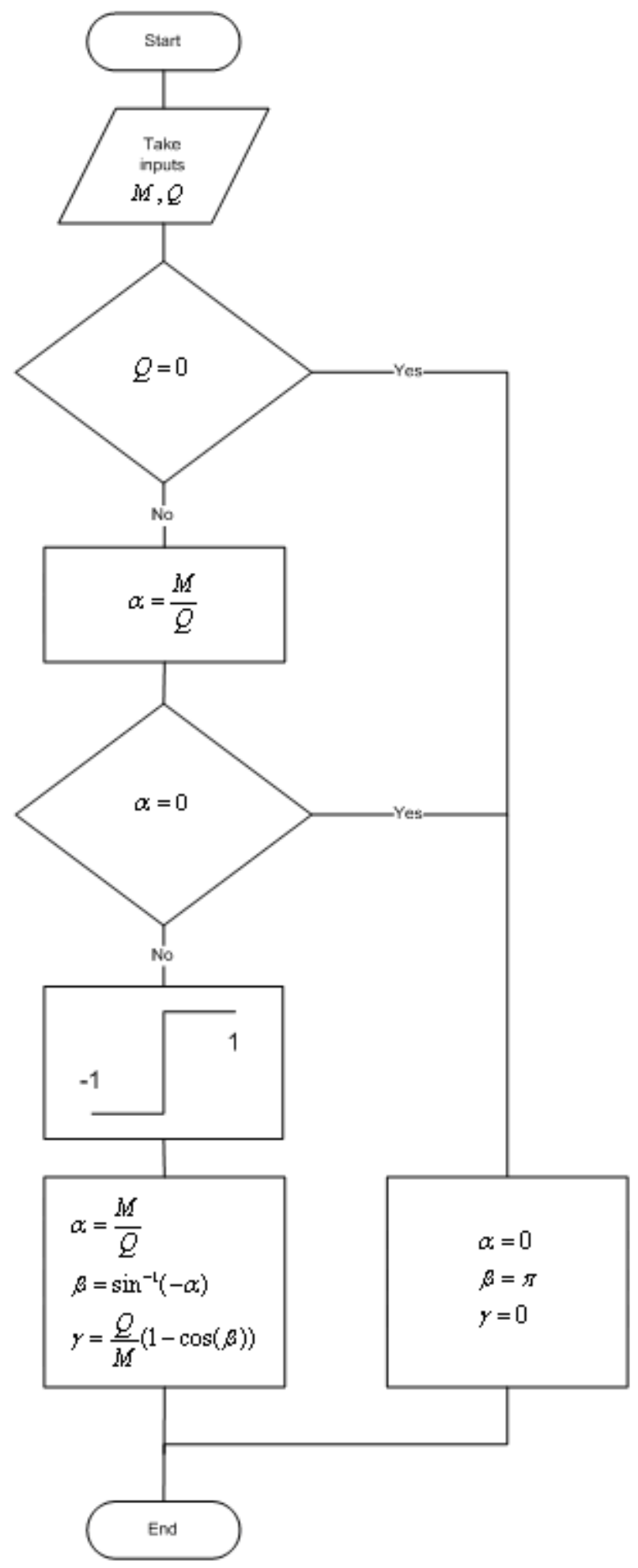

Figure 2.20: Alpha, Beta and Gamma block flowchart 
One solution to the imaginary value problem is to limit the input to $S I N^{-1}$ function to values between -1 and 1 since the $S I N^{-1}$ function generates real numbers in this range. The block was tested again with the limiter but it still keeps running into convergence problems. After testing the block with the limiter function there was a big question about the functionality of the Pspice $S I N^{-1}$ function. It was decided to build a new $S I N^{-1}$ function with added conditions to avoid convergence problems.

\subsubsection{Building Inverse Sine function in Pspice}

A popular way to rebuild complex non-linear functions like inverse sine or $S I N^{-1}$ is by using a series expansion to represent the function around certain point $\mathrm{x}$. Series expansion is a representation of a particular function as a sum of powers in one of its variables. The most famous series expansion method is the Taylor series defined in Equation 2.21. If $a=0$ the series is called the Maclaurin series. The Maclaurin series is a Taylor series expansion of a function about 0 . The general form of the Maclaurin series is given in Equation 2.22 [21, 22, 23].

$$
\begin{gathered}
f(x)=\sum_{n=0}^{\infty} \frac{f^{(n)}(a)}{n !}(x-a)^{n} \\
f(x)=f(0)+f^{\prime}(0) x+\frac{f^{\prime \prime}(0)}{2 !} x^{2}+\frac{f^{(3)}(0)}{3 !} x^{3}+\cdots+\frac{f^{(n)}(0)}{n !} x^{n}+\cdots
\end{gathered}
$$

Using the Maclaurin series, the general form of Inverse Sine function or $\operatorname{SIN}^{-1}$ is given in equation 2.23. 


$$
\operatorname{SIN}^{-1}(x)=\sum_{n=0}^{\infty} \frac{\Gamma\left(n+\frac{1}{2}\right)}{\sqrt{\pi}(2 n+1) n !} x^{2 n+1}
$$

Where:

$$
\begin{aligned}
\Gamma(x) & =\int_{0}^{\infty} t^{x-1} e^{-t} d t \\
& =\left[-t^{x-1} e^{-t}\right]_{0}^{\infty}+\int_{0}^{\infty}(x-1) t^{x-2} e^{-t} d t \\
& =(x-1) \int_{0}^{\infty} t^{x-2} e^{-t} d t \\
& =(x-1) \Gamma(x-1) .
\end{aligned}
$$

If $\mathrm{x}$ is an integer:

$$
\begin{aligned}
\Gamma(n) & =(n-1) \Gamma(n-1) \\
& =(n-1)(n-2) \Gamma(n-2) \\
& =(n-1)(n-2)(n-3) \cdots 1 \\
& =(n-1) !
\end{aligned}
$$

One issue during the implementation of $S I N^{-1}$ function is choosing a good value of " $n$ " that satisfies both accuracy and the adequate number of blocks for Pspice implementation. Using different values for " $n$ ", different approximation curves were obtained. Those curves were compared with actual values of $S I N^{-1}$ which were obtained by using a large value for "n". Figure 2.21 shows the different approximation curves versus the actual inverse sine function. From the figure it was noted that choosing $n=15$ gives a very high approximation accuracy for input values $\mathrm{x}$ less than 0.9 and an error of $1 \%$ for $x$ values larger than 0.9 . This error figure remain almost the same until $n=23$. 


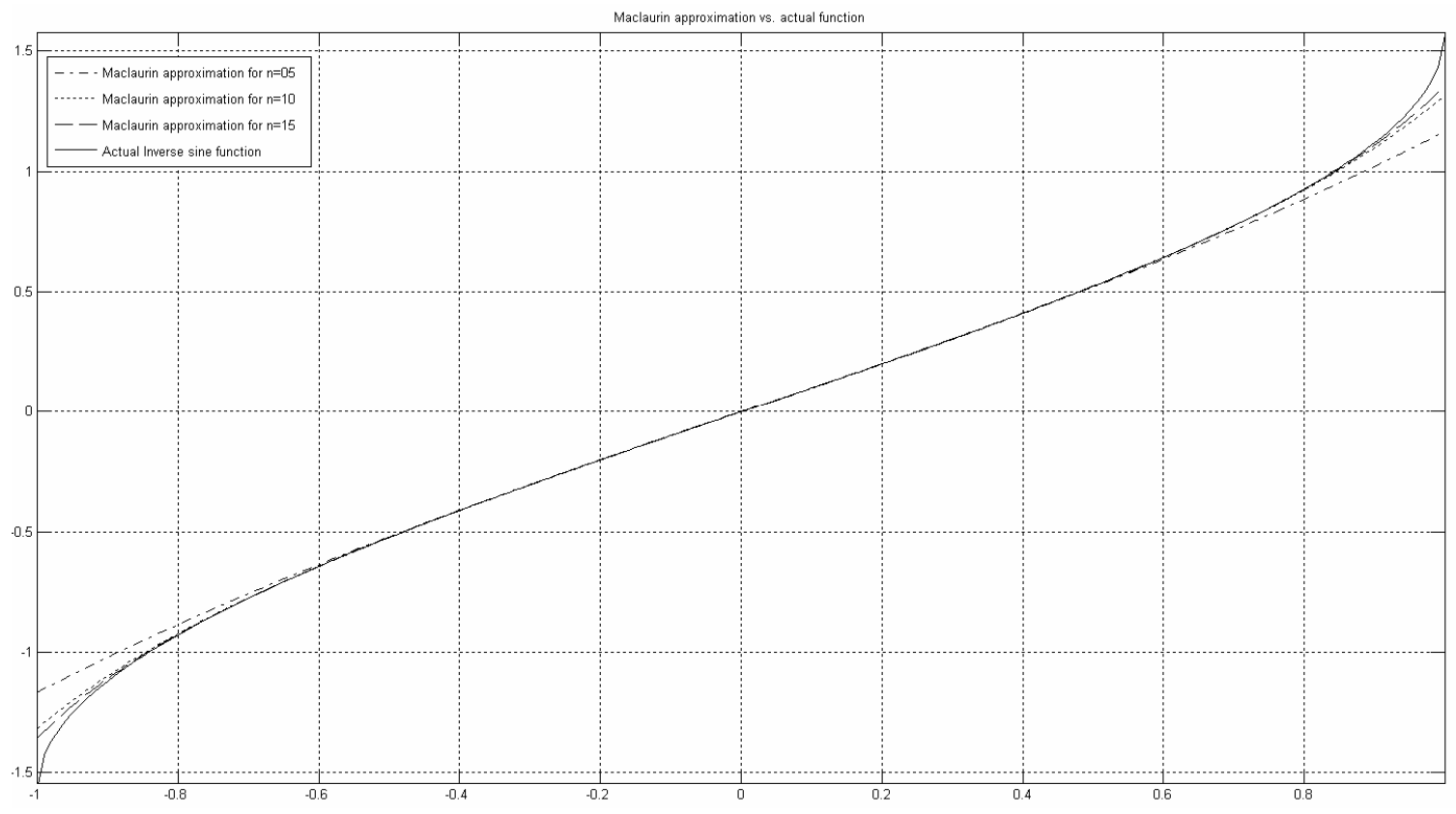

Figure 2.21: Maclaurin series approximation versus actual $S I N^{-1}$ function

Using the Block-Concatenation technique, the development procedure for $S I N^{-1}$ block is shown in Table 2.5. Based on this procedure, a flowchart was developed to implement the $S I N^{-1}$ block. Figure 2.22 shows the flowchart.

Table 2.5: development procedure for $S I N^{-1}$ block

\begin{tabular}{|c|l|c|}
\hline Procedure & & \\
\hline 1 & Equation Input & $x$ in the range $[-1,1]$ \\
\cline { 2 - 3 } & Equation Output & $\operatorname{SIN}^{-1}(x)$ \\
\hline 2 & $\begin{array}{l}\text { Mathematical and } \\
\text { Logical Expressions }\end{array}$ & $\begin{array}{c}\text { Addition,Multiplication } \\
\text { and Division }\end{array}$ \\
\hline 3 & Required Sub-Blocks & $\begin{array}{c}\text { Eight } A B M \text { blocks to } \\
\text { represent the Maclaurin } \\
\text { series coefficients. }\end{array}$ \\
\hline
\end{tabular}




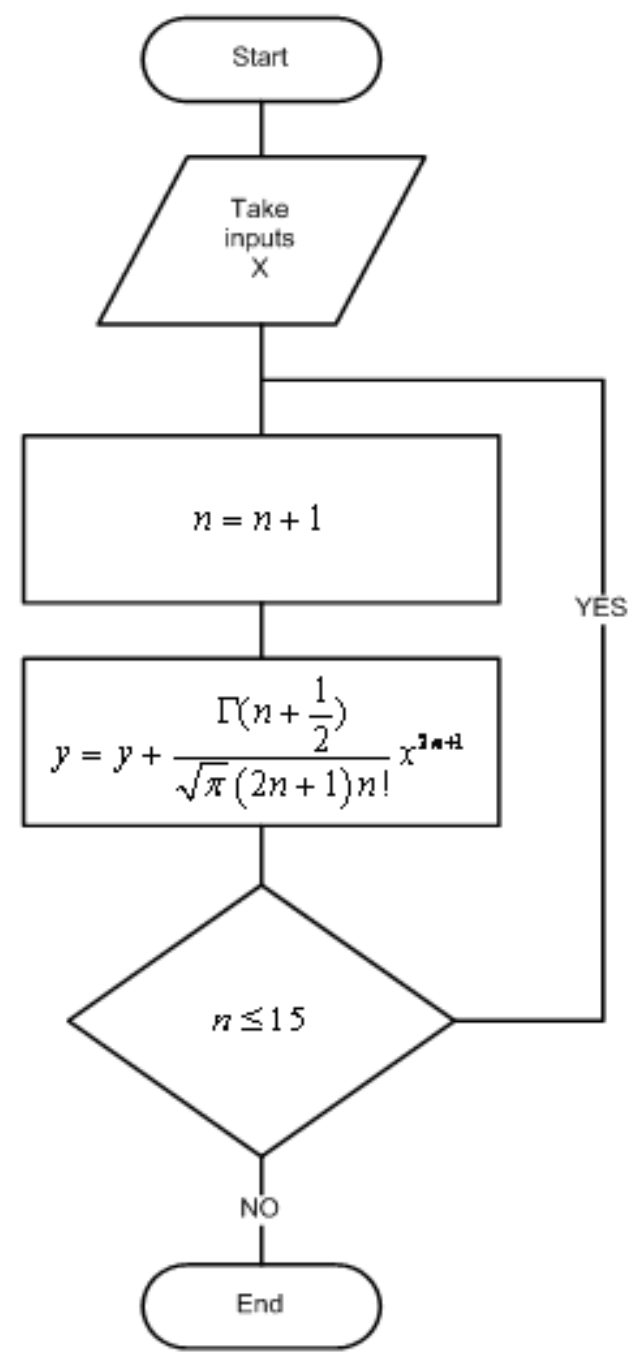

Figure 2.22: $S I N^{-1}$ block flowchart

Figure 2.23 shows the Pspice implementation of BG block along with the Maclaurin series implementation of $S I N^{-1}$. One note about Pspice implementation is that ABM block has maximum capacity of 132 characters. This limits the capability of programming all the Maclaurin coefficients in one line. The problem was solved by spreading the coefficients through many blocks by using the Block concatenation technique. 


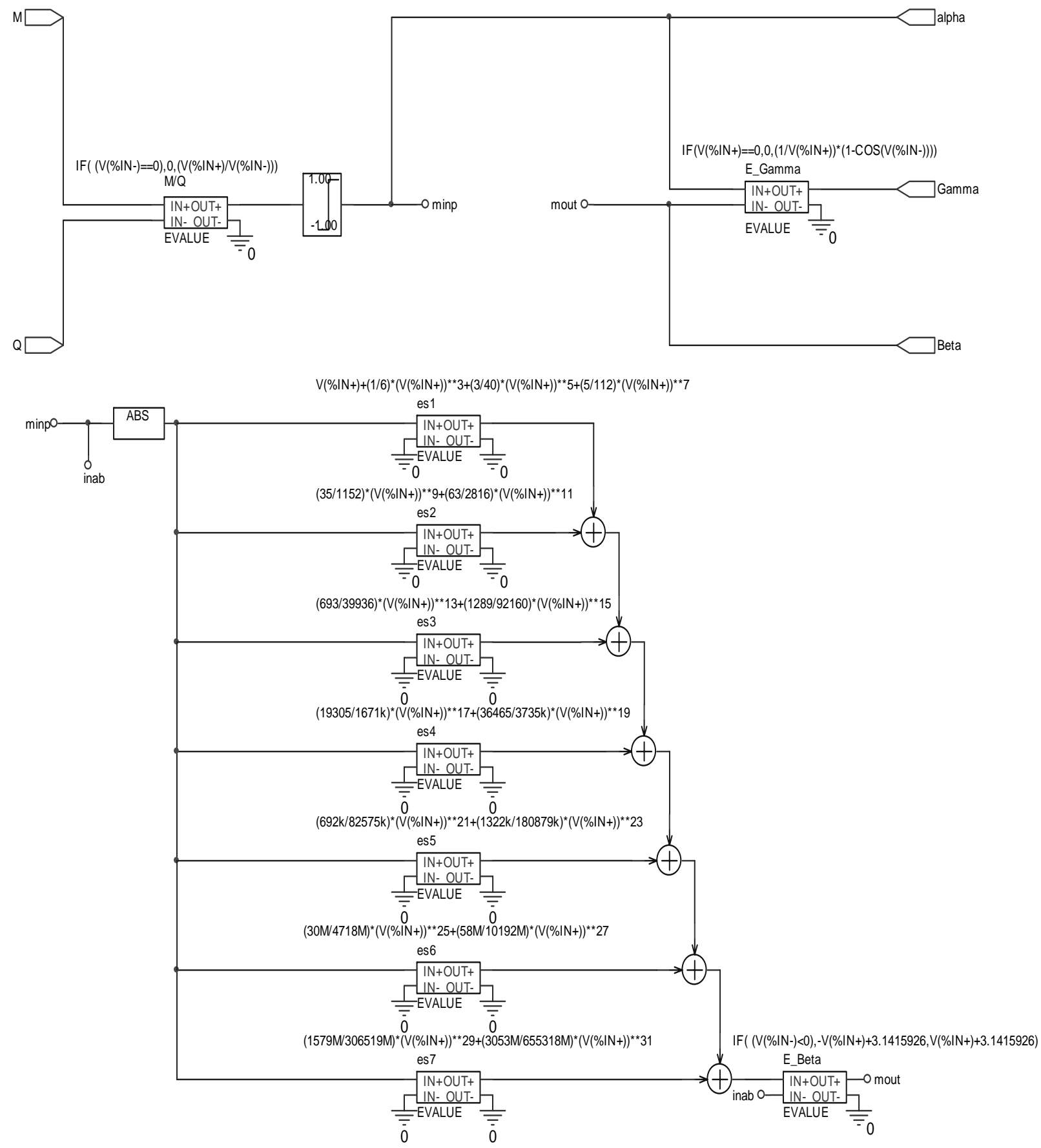

Figure 2.23: Pspice implementation of $B G$ Block 


\subsubsection{Error Block “ERR Block”}

Error block, or ERR Block, is the block responsible for calculating the error value for the unified model. The error value is the absolute difference between the true $f_{n s}$ value obtained from the normalized switching frequency block (Section 2.6.2) and the calculated $f_{n s}$ value obtained from Equation 2.13. Error value is an important quantity used to set the conditional statement which determines the continuation or breaking out from the steady-state solution loop (as will be discussed in Section 2.6.6). Figure 2.24 shows the ERR block.

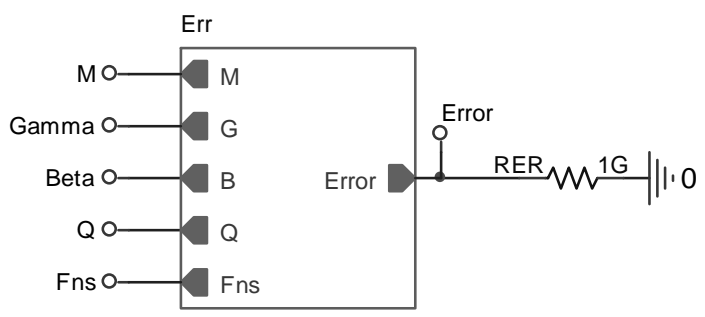

Figure 2.24: Error Block

The ERR Block represents the second type of relation between the Unified Model blocks. The first type was discussed in system architecture Section 2.5 , where the information is exchanged between the main block and sub-blocks. In the second type, the sub-blocks exchange information between each other. In this type of relation, each block calculates its own outputs and those outputs can be passed to other blocks without returning back to the main block. The ERR Block has both types of relation. As shown in Figure 2.24, the ERR block takes the outputs of the BG Block (Alpha, Beta and Gamma) and the normalized switching frequency block ( $f_{n s}$ value). This is a sub-block to sub- 
block relation. In the other type, the ERR block exchanges data with the SOL block (Section 2.6.6, Error value and steady-state solution). This is sub-block to main-block relation.

The ERR Block is a direct implementation of the normalized switching frequency equation developed in Section 2.3. This equation is repeated here as a reference for the ERR block flowchart.

$$
f_{n s}=\frac{-2 \pi M}{\frac{M}{2 Q} \gamma^{2}-(\beta+\gamma-\sin \beta-\gamma \cos \beta)}
$$

Using the Block-Concatenation technique, the development procedure for the $E R R$ block is shown in Table 2.6. Based on this procedure, a flowchart was developed to implement the ERR block. Figure 2.25 shows the flowchart.

Table 2.6: development procedure for ERR block

\begin{tabular}{|c|l|c|}
\hline Procedure & & \\
\hline 1 & Equation Inputs & $M, \beta, \gamma, Q, f_{n s}$ \\
\cline { 2 - 3 } & Equation Output & Error value \\
\hline 2 & $\begin{array}{l}\text { Mathematical and } \\
\text { Logical Expressions }\end{array}$ & $\begin{array}{l}\text { Addition,Multiplication } \\
\text { and Division }\end{array}$ \\
\hline 3 & Required sub-blocks & $\begin{array}{c}\text { The implementation of } \\
\text { Equation (2.13) was } \\
\text { divided between five } \\
\text { blocks and the sixth } \\
\text { block was used to } \\
\text { calculate the absolute } \\
\text { error. }\end{array}$ \\
\hline
\end{tabular}




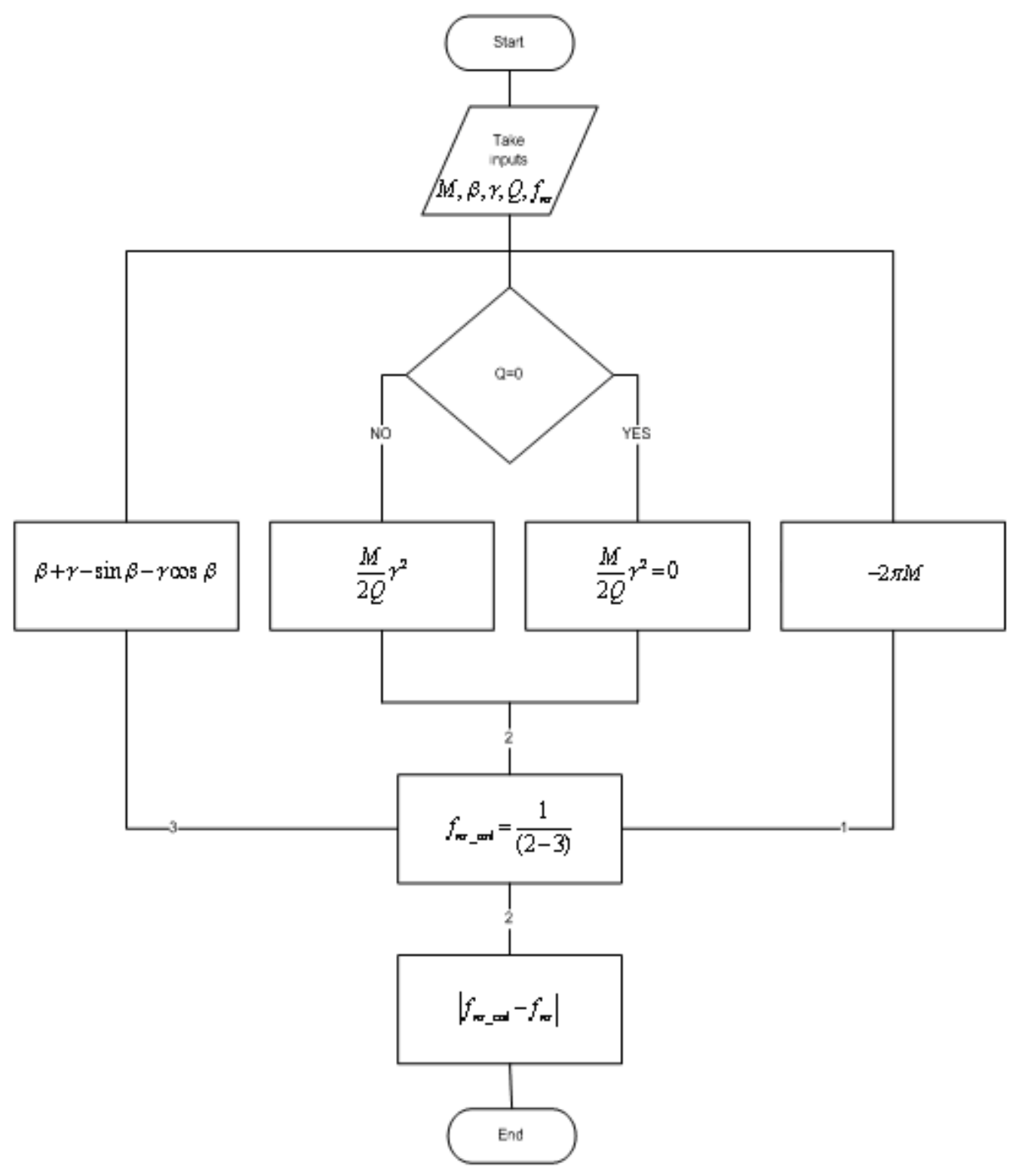

Figure 2.25: The ERR block flowchart

The flowchart was converted at later stage into functional Pspice block. Figure 2.26 shows the Pspice implementation of the Error or ERR block. The block was tested in Pspice and worked successfully. To get a better understanding of the results, the simulation figures were included in the SOL block (Section 2.6.6). 


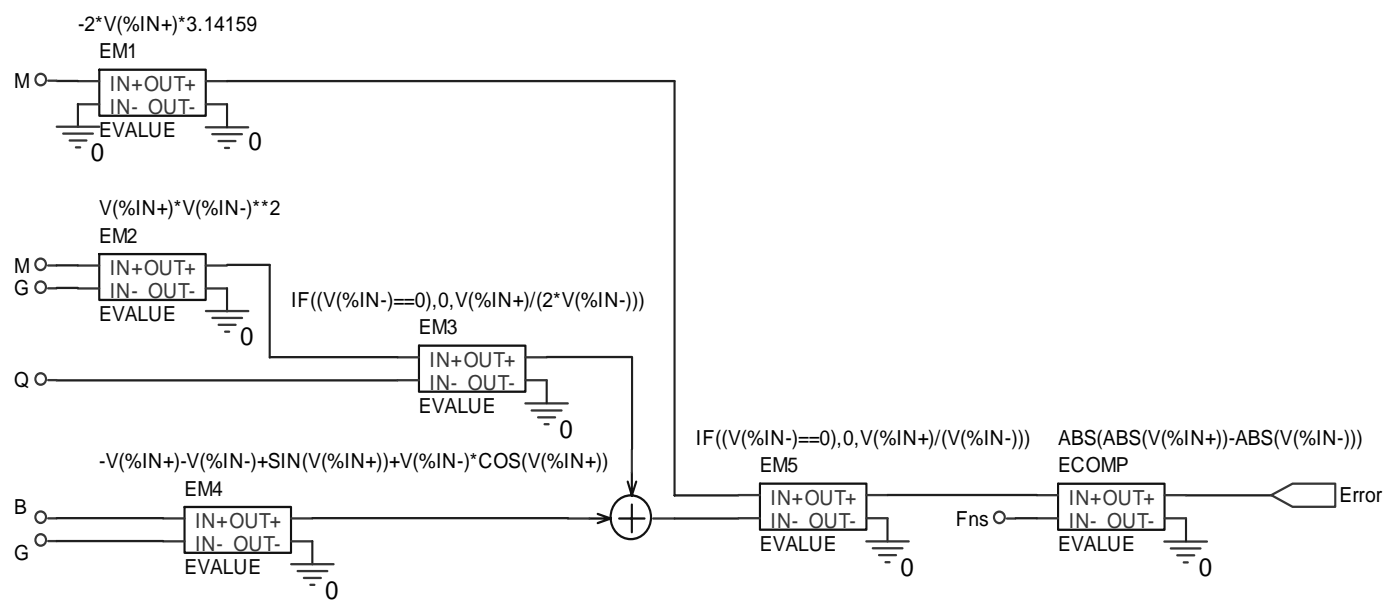

Figure 2.26: Pspice implementation of the ERR Block

\subsubsection{Steady-State Gain Solution Block “SOL Block”}

Steady-State gain Solution, or SOL Block, is the block responsible for calculating the Steady-State gain value for the unified model. The Steady-State gain value is the ratio of the converter output voltage to the converter input voltage. The Steady-State gain solution $M$ is the final product of the Unified Model that is used by other sub-blocks to generate the state-plane diagram and the Steady-State waveforms for the switching cell inductor current $I_{L r}$ and switching cell capacitor voltage $V_{C r}$. Figure 2.27 shows the $S O L$ block.

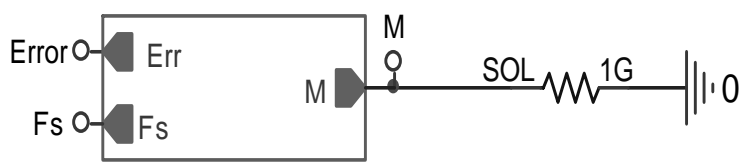

Figure 2.27: The Steady-State Gain Solution Block 
The basic logic behind the SOL Block is that is has a FOR loop. In this loop the Steady-State gain value $M$ starts from a very low value and keeps increasing iteratively. For each new value of $M$, a new error value is generated by the ERR Block (Section 2.6.5). Then this error is compared with a predetermined threshold. If the calculated error is below that threshold, the system breaks out from the loop and outputs the latest calculated Steady-State gain value $M$. Implementing the SOL Block was challenging for many reasons: first, determining when to break out from the loop and the correct way to do it. Second, the SOL Block has to respond to changes in the switching frequency as going to be discussed in the next chapter. To understand this block thoroughly, the block is divided into sub-blocks where each sub-block is explained independently and finally their combined function is illustrated.

\subsubsection{FOR Loop with Breakout capability}

As discussed earlier in this section, the unified Steady-State gain solution is found by going through several iterations until the error value goes below a certain threshold. Then the iteration process stops and the final calculated value is generated. To implement this process, a FOR loop with breakout capability is needed. The implementation of a generic FOR loop was discussed in Section 2.4.2.1 "Creating FOR Loop." Briefly, FOR loop is a simple ramp source that, in this case, goes from 0 to a user specified value, and the condition is the monitored error signal; if the error value goes below certain threshold, the loop is terminated. 
Figure 2.28 shows the basic implementation of the SOL Block structure. The block works as follows: The ABM block keeps monitoring the error signal generated from ERR Block. While the error signal is greater than the threshold value, the Sbreak switch is $\mathrm{ON}$ and the capacitor is charging, The Sbreak switch is programmed to turn ON when the control signal equals 1 volt, and to turn OFF when the control signal equals -1 volt. Once the error signal goes below a 0.01 threshold, a -1 volt control signal is generated from the ABM block to turn the Sbreak OFF switch and to stop charging the capacitor. At this time the capacitor holds the last charged value.

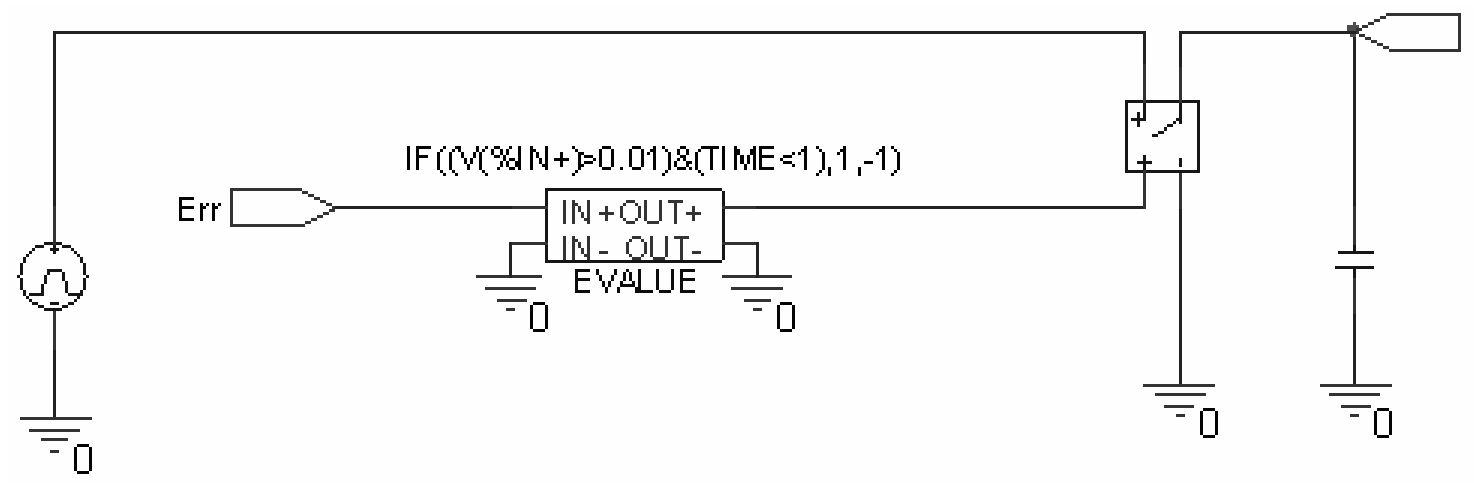

Figure 2.28 Basic implementation of SOL Block structure

The block in this simple structure cannot work correctly, since the error signal converges toward a solution - if there is any - and then starts diverging again. Figure 2.29 illustrates the problem more clearly. 


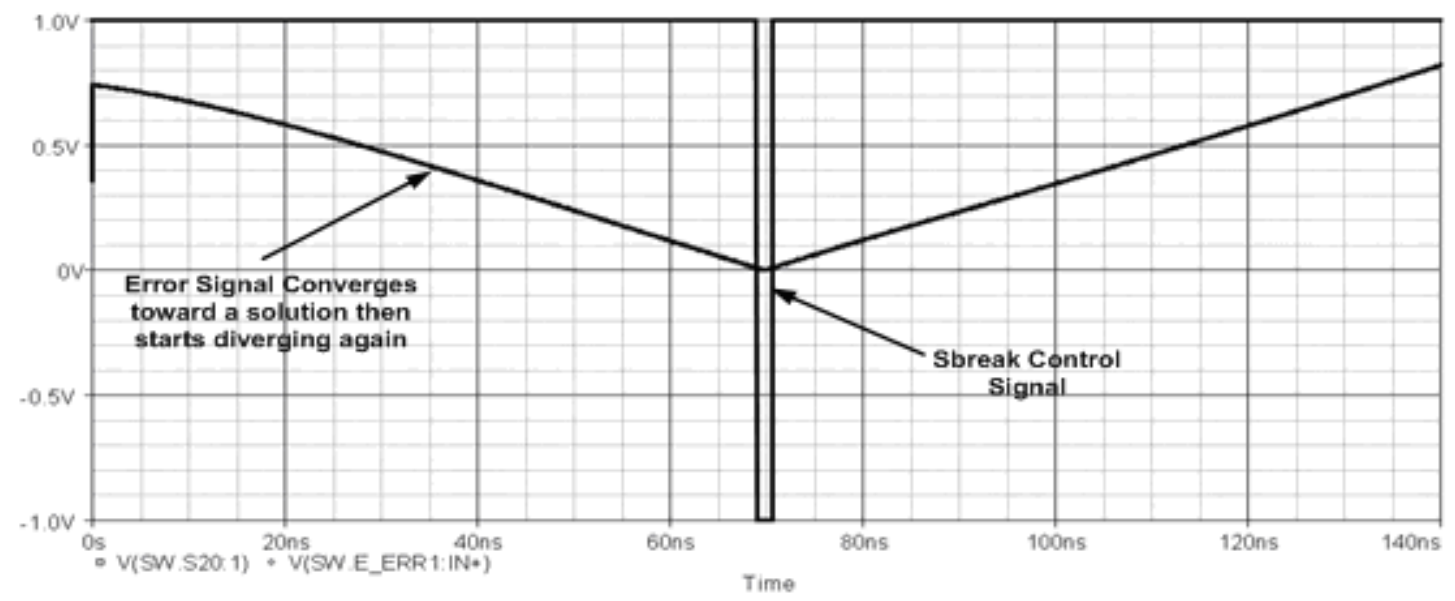

Figure 2.29 Error Signal Behavior

In Figure 2.29, The Sbreak Control signal which controls the charging of the Capacitor is $\mathrm{ON}$ and the capacitor is charging as long as the error value is above the predetermined thresholds. When the error reaches the threshold value, the Sbreak signal is OFF and the capacitor holds the latest charged value. But since the error will diverge again, the error signal will no longer be below the threshold value, and thus the Sbreak control signal will turns $\mathrm{ON}$ again. Hence the capacitor will start charging again and the Steady-State solution will be lost. One solution to this problem would be adding a latch to the control signal. That is, once a solution is achieved the control will be latched to the OFF state, whatever changes happen at a later time. Figure 2.30 shows the proposed circuit. 


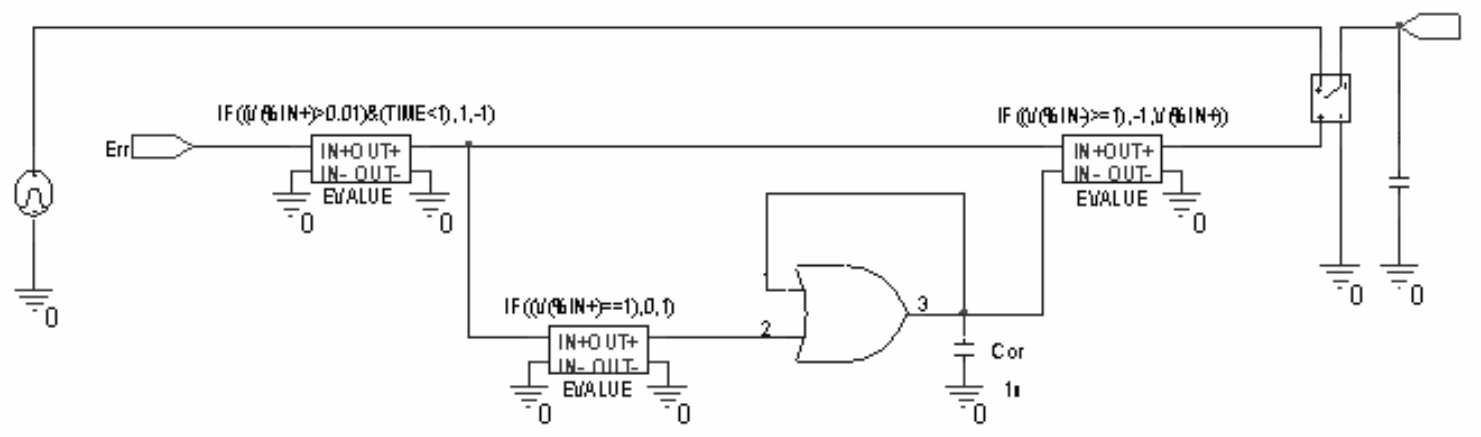

Figure 2.30 SOL Block with a latch circuit

From Figure 2.30, the OR gate is used as a Memory element. The output of the OR gate is fed back to one of its inputs. A capacitor with zero initial voltage is added at the output of the OR gate to set the gate initial conditions to zero. The logic of the circuit can be explained as follows: while the error is greater than the threshold value, the ABM block E_ERR1 outputs a logic zero to the input of the OR gate. The other input is zero since it is connected to the output Capacitor $C_{O R}$, which has a zero initial value. This means the output of the OR gate is zero. Now, once a solution is achieved, the ABM block E_ERR1 outputs a logic one to the input of the OR gate. This means the OR gate output will be logic one. It should be noted here that the OR gate now acts as a memory element that stores this logic 1 value, since, regardless of the input to the OR gate, its output will always be logic 1. Finally, the ABM block E_ERR4 monitors the output of the OR gate; if the OR gate output changes to logic 1, the E_ERR4 block forces the Sbreak switch to turn OFF. And hence the found solution will be stored. The SOL Block with the latch circuit in Figure 2.30 was tested with the same error signal shown in Figure 2.29. The outputs of the circuit are shown in Figure 2.31. From the figure, the ramp source is charging the capacitor and the capacitor has the same voltage as the ramp 
source. The control signal has a high value and hence the switch is ON. At certain times, the error goes below the error threshold value. The control signal will have a low value, hence the switch is OFF and the capacitor holds the latest charged value.

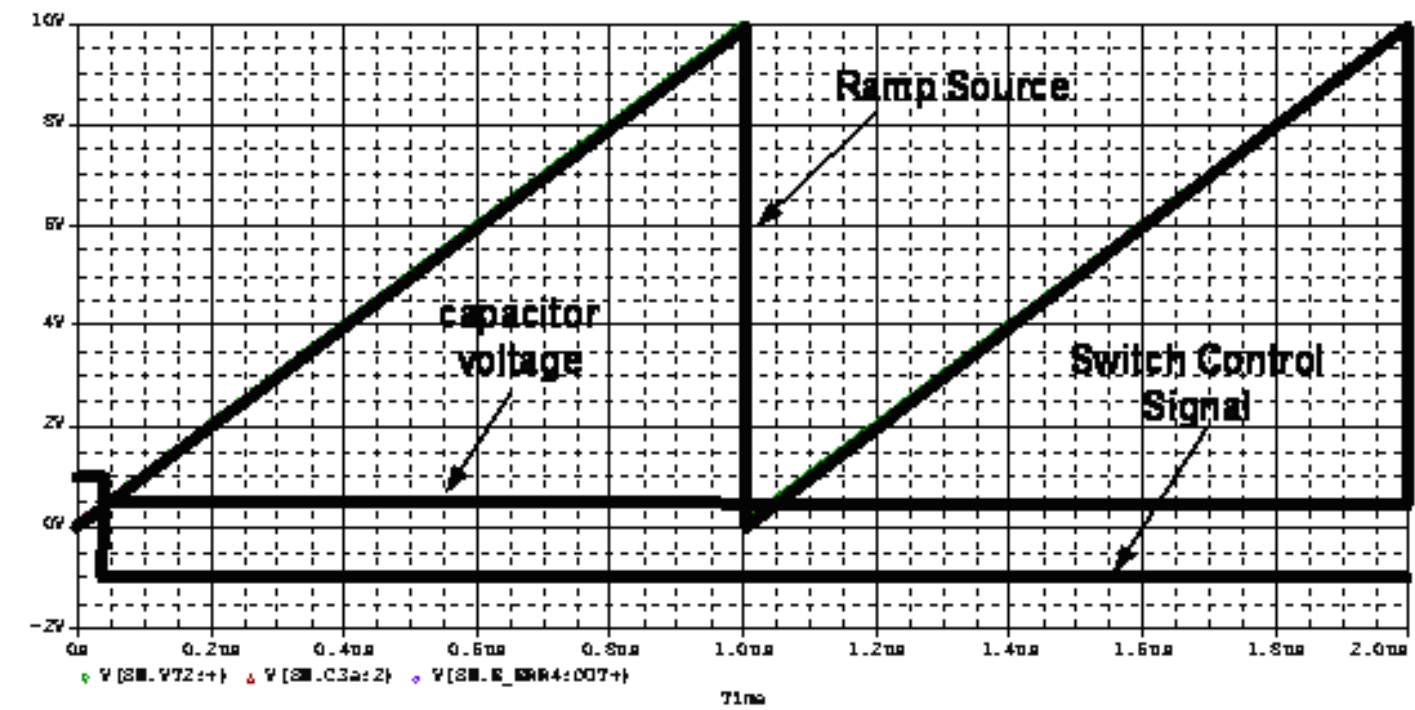

Figure 2.31 SOL Block with a latch circuit outputs 


\section{CHAPTER 3}

\section{UNIFIED MODEL APPLICATIONS}

\subsection{Introduction}

The unified steady-state model is a new way to simulate soft-switching cells; the user connects the simulation model to the desired topology configuration and the Model calculates the steady-state gain solution for the user. The model also provides the user with different plots needed for soft-switching cell design. These include the internal switching cell information such as switching cell inductor current $I_{L r}$ and the switching cell capacitor voltage $V_{C r}$, and their corresponding state-plane diagram. The model can also be used in feedback design applications where the output voltage is regulated by changing the switching frequency.

This chapter includes a detailed discussion about how the above applications were included in the unified model along with their Pspice implementation. Section 3.2 discusses how the unified model generates the switching cell inductor current $I_{L r}$ and the

switching cell capacitor voltage $V_{C r}$ steady-state waveform. Section 3.3 presents the State-Plane Diagram using the unified model. Section 3.4 shows feedback design using the unified model and, finally, section 3.5 shows how the model can be used with other topologies. 


\subsection{Generating $I_{L r}$ and $V_{C r}$ steady-state waveform}

The steady-state plot for the switching cell inductor current $I_{L r}$ and the switching cell capacitor voltage $V_{C r}$ includes important waveforms that show the user how the soft switching cell behaves at different times and if the soft switching condition was achieved. Also, the waveforms are useful in determining the voltage and current stress on the switch.

$I_{L r}$ and $V_{C r}$ steady-state waveforms can be generated using the $D R W$ block. The $D R W$ block is the block responsible for generating the time domain steady-state waveforms of the soft-switching cell inductor current and the soft-switching cell capacitor voltage. Figure 3.1 shows the $D R W$ block [12].

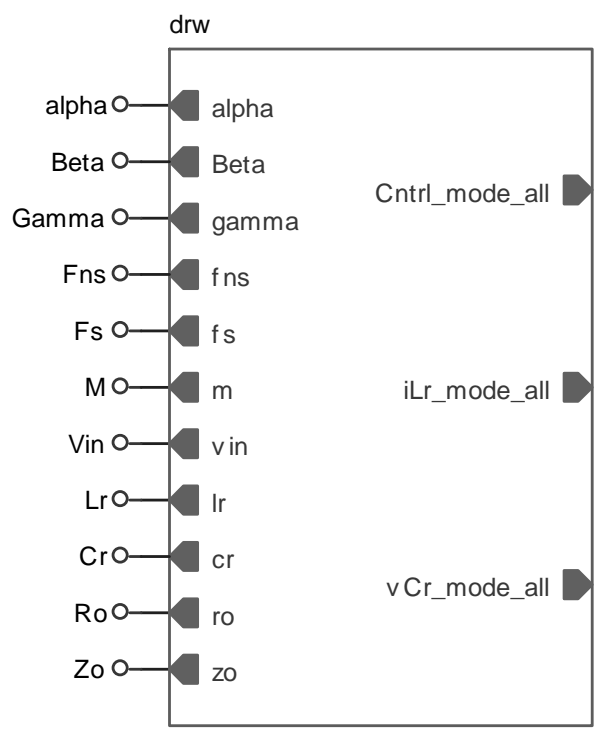

Figure 3.1: $I_{L r}$ and $V_{C r}$ steady-state waveform Block 
The $D R W$ Block generates the time domain waveforms by using the softswitching cell equations derived for different modes of operations; in other words, the $D R W$ block has different time ranges and for each time range there is a specific equation that describes the switching cell capacitor voltage and the switching cell inductor current. If the simulation time is within that range, the corresponding equation will be used and the other equations will be equated to zero. At the end, the results from different time intervals will be added. Based on the analysis in Section 1.5, the zero current switching (ZCS) quasi-resonant converter (QRC) has four modes of operation. Based on those modes, the $D R W$ block has four sub-blocks that use the steady-state equations for different modes to generate the time domain waveforms.

Using the Block-Concatenation technique, the development procedure for the $D R W$ block is shown in Table 3.1. Based on this procedure, a flowchart was developed to implement the $D R W$ block. Figure 3.2 shows the flowchart.

Table 3.1: development procedure for $D R W$ block

\begin{tabular}{|c|c|c|}
\hline Procedure & & \\
\hline 1 & $\begin{array}{c}\text { Equation } \\
\text { Inputs }\end{array}$ & $\alpha, \beta, \gamma, f_{n s}, f_{s}, M, V_{\text {in }}, L_{r}, C_{r}, R_{o}, Z_{o}$ \\
\cline { 2 - 3 } & $\begin{array}{c}\text { Equation } \\
\text { Outputs }\end{array}$ & $I_{L r} \& V_{C r}$ Steady-state waveforms \\
\hline 2 & $\begin{array}{c}\text { Mathematical } \\
\text { and Logical } \\
\text { Expressions }\end{array}$ & $\begin{array}{c}\text { Addition, Multiplication and } \\
\text { Division }\end{array}$ \\
\hline 3 & $\begin{array}{c}\text { Required } \\
\text { sub-blocks }\end{array}$ & $\begin{array}{c}\text { Four sub-blocks to implement the } \\
\text { four different modes of operation }\end{array}$ \\
\hline
\end{tabular}




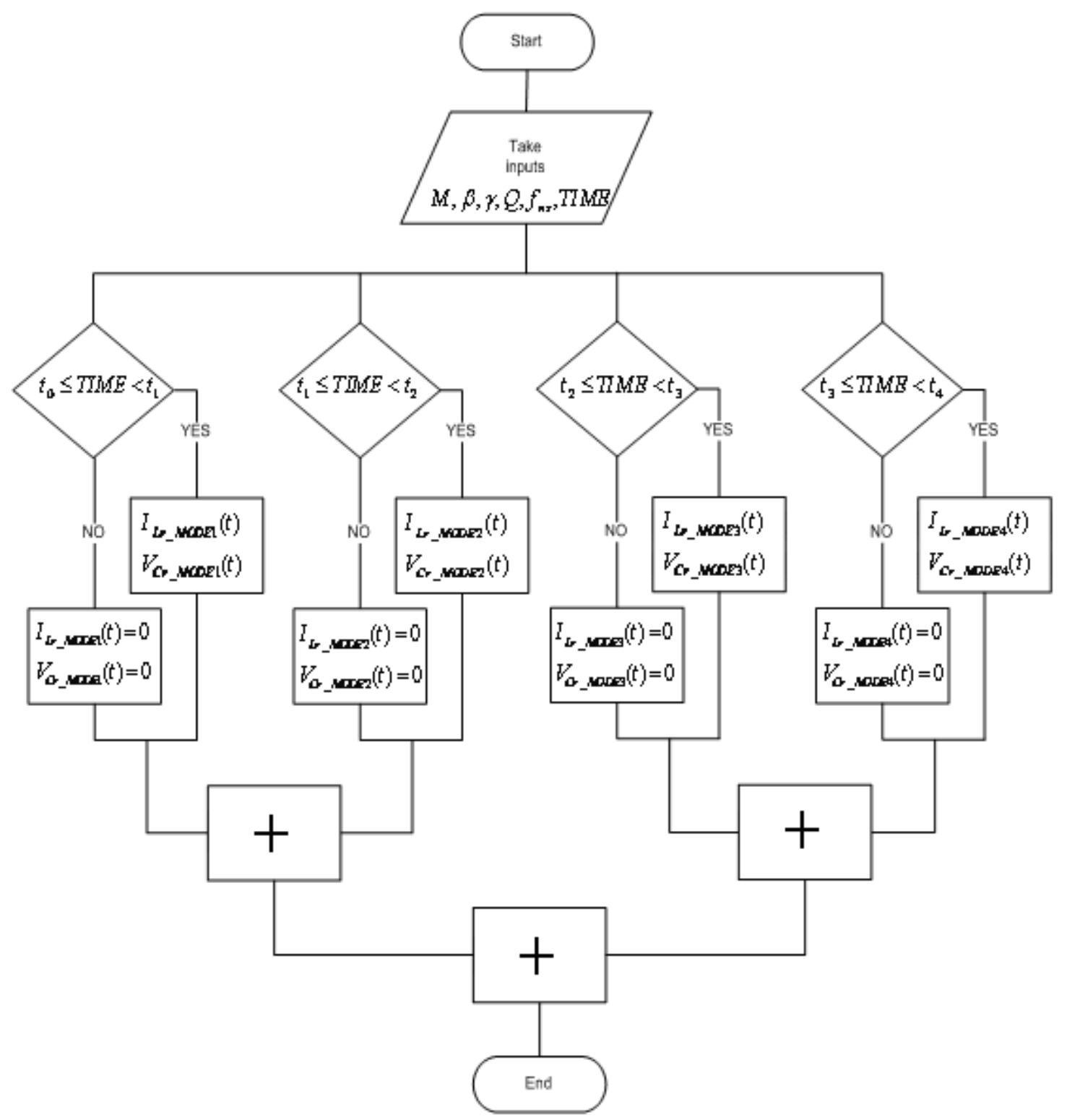

Figure 3.2: $D R W$ block flowchart

The following paragraphs summarize the ZCS-QRC four modes of operations and their corresponding Pspice blocks. The derivation for the equations below was previously introduced in Chapter 1, Section 1.5. 
Mode 1: $\left[t_{o} \leq t \leq t_{1}\right]$ :

This mode starts at time $t=t_{0}$ when the switch turns ON, and ends at time $t=t_{1}$ when the switching cell diode D turns off. The following equations summarize this mode:

$$
\begin{gathered}
v_{c r}(t)=0 \\
i_{L r}(t)=\frac{V_{g}}{L_{r}}\left(t-t_{0}\right) \\
v_{C r}\left(t_{1}\right)=0 \\
i_{L r}\left(t_{1}\right)=I_{F}
\end{gathered}
$$

The corresponding Pspice block for this mode is shown in Figure 3.3, where the block represents a direct implementation of the above equations.

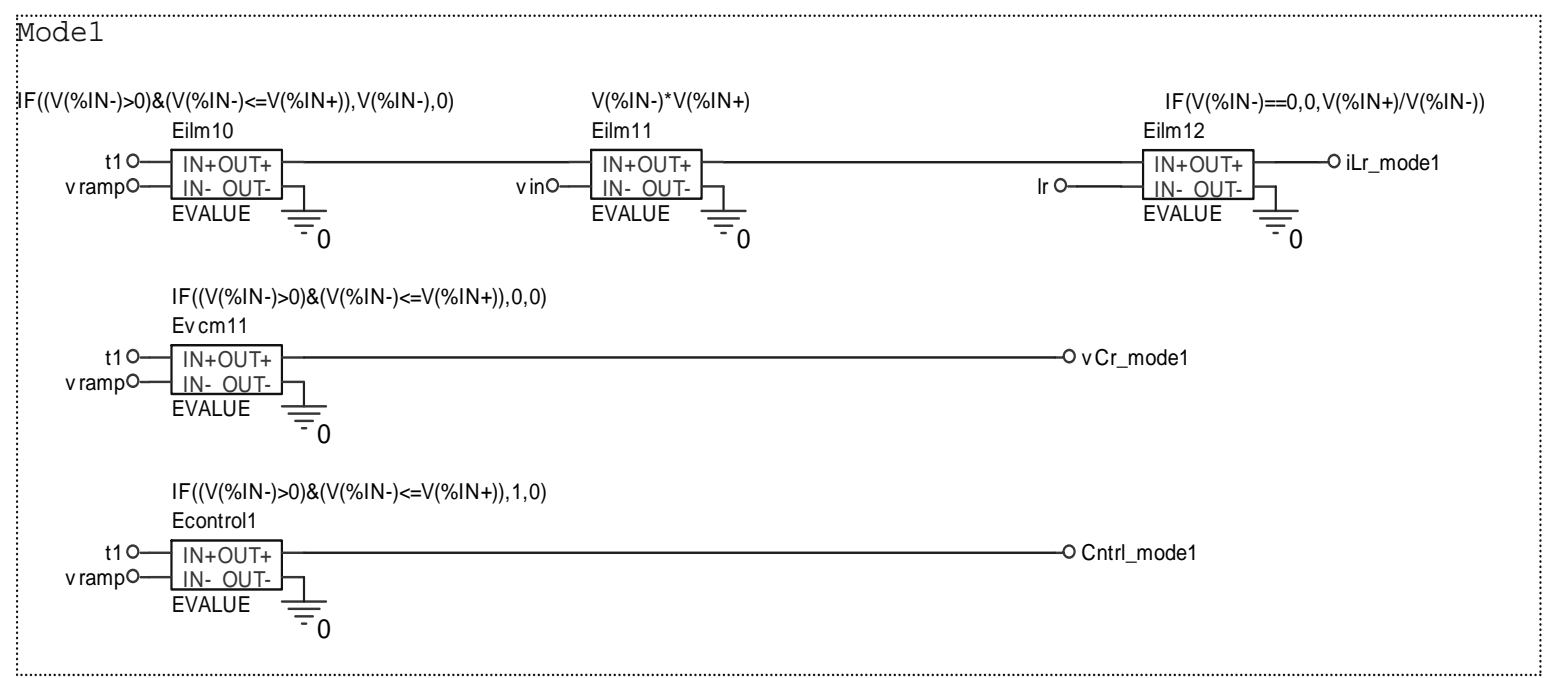

Figure 3.3: $M O D E 1$ Pspice block 
Mode 2: $\left[t_{1} \leq t \leq t_{2}\right]$ :

This mode starts at time $t=t_{1}$ when the Diode $D$ turns OFF, and ends at time $t=t_{2}$ when the current through $L_{r}$ drops to zero. The following equations summarize this mode:

$$
\begin{gathered}
v_{C r}(t)=V_{g}\left[1-\cos w_{0}\left(t-t_{1}\right)\right] \\
i_{L r}(t)=I_{F}+\frac{V_{g}}{Z_{o}} \sin w_{0}\left(t-t_{1}\right) \\
i_{L r}\left(t_{2}\right)=0
\end{gathered}
$$

The corresponding Pspice block for this mode is shown in Figure 3.4, where the block represents a direct implementation of the above equations.

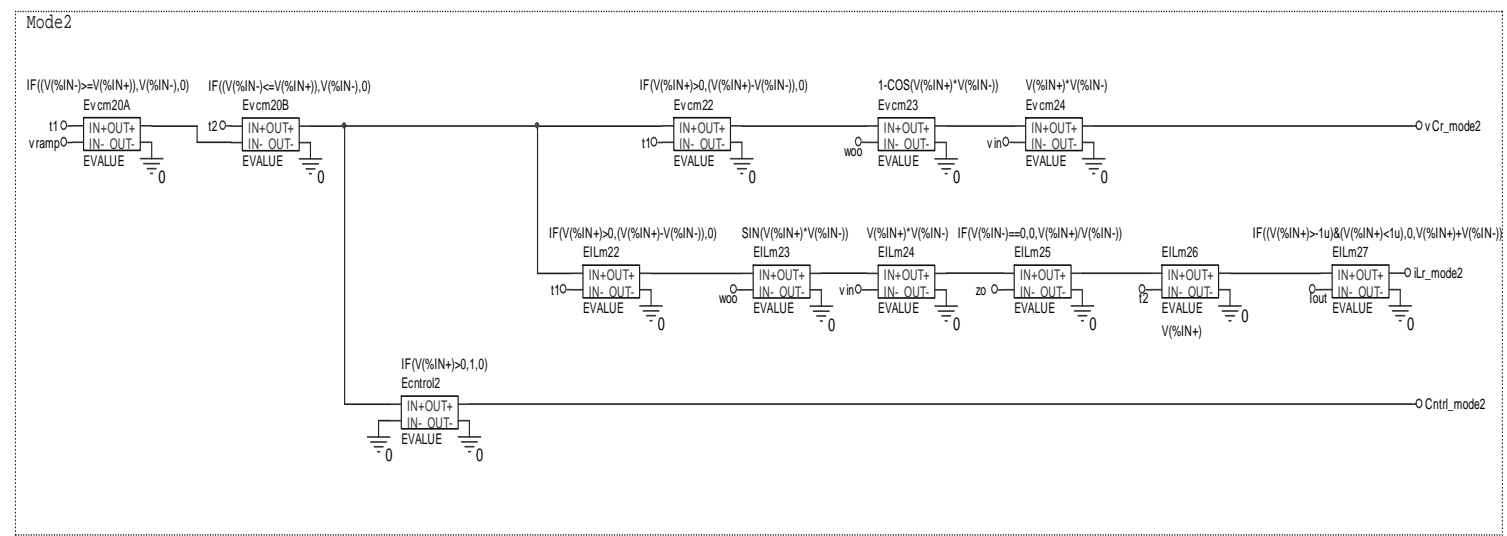

Figure 3.4: $M O D E 2$ Pspice block 
Mode 3: $\left[t_{2} \leq t \leq t_{3}\right]$ :

This mode starts at time $t=t_{2}$ when the switch $S$ is turned OFF, and ends at time $t=t_{3}$ when the capacitor voltage drops to zero causing the diode $D$ to turn ON. The following equations summarize this mode:

$$
\begin{gathered}
v_{C r}(t)=-\frac{I_{F}}{C_{r}}\left(t-t_{2}\right)+V_{g}[1-\cos \beta] \\
i_{L r}(t)=0 \\
v_{C r}\left(t_{3}\right)=0
\end{gathered}
$$

The corresponding Pspice block for this mode is shown in Figure 3.5, where the block represents a direct implementation of the above equations.

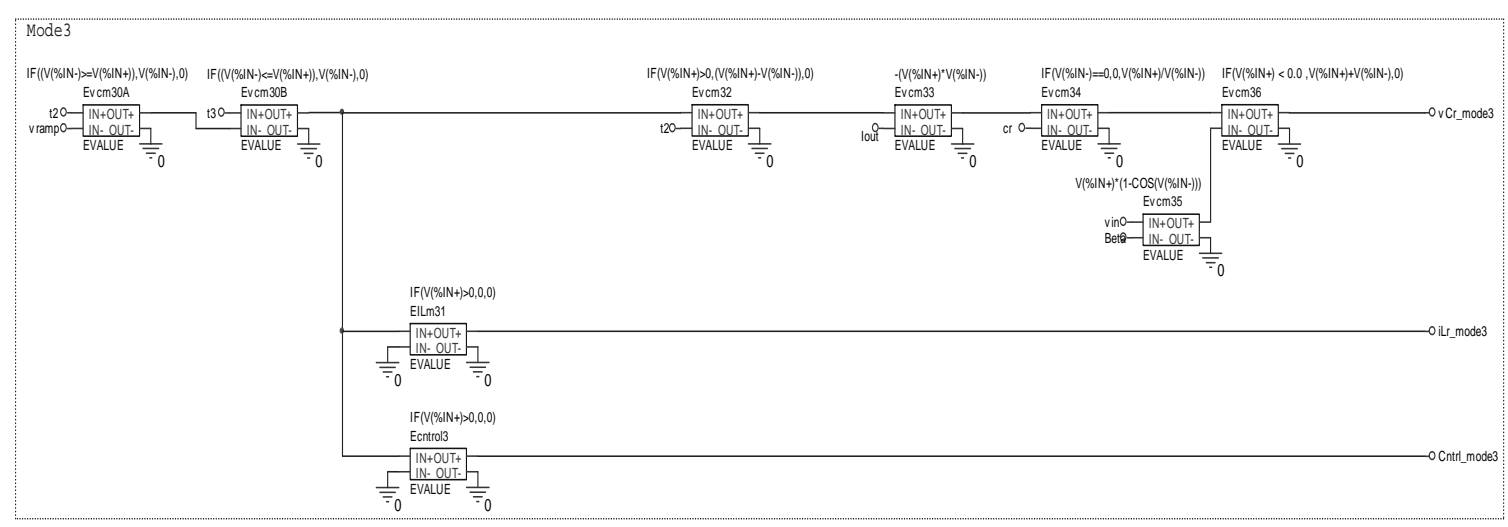

Figure 3.5: MODE3 Pspice block 
Mode 4: $\left[t_{3} \leq t \leq t_{o}+T_{s}\right]$ :

This mode is the steady-state mode and nothing happens until the switch $S$ turns ON again to start the next switching cycle. The following equations summarize this mode:

$$
\begin{gathered}
i_{L r}(t)=0 \\
v_{C r}\left(t_{3}\right)=0
\end{gathered}
$$

The corresponding Pspice block for this mode is shown in Figure 3.6, where the block represents a direct implementation of the above equations.

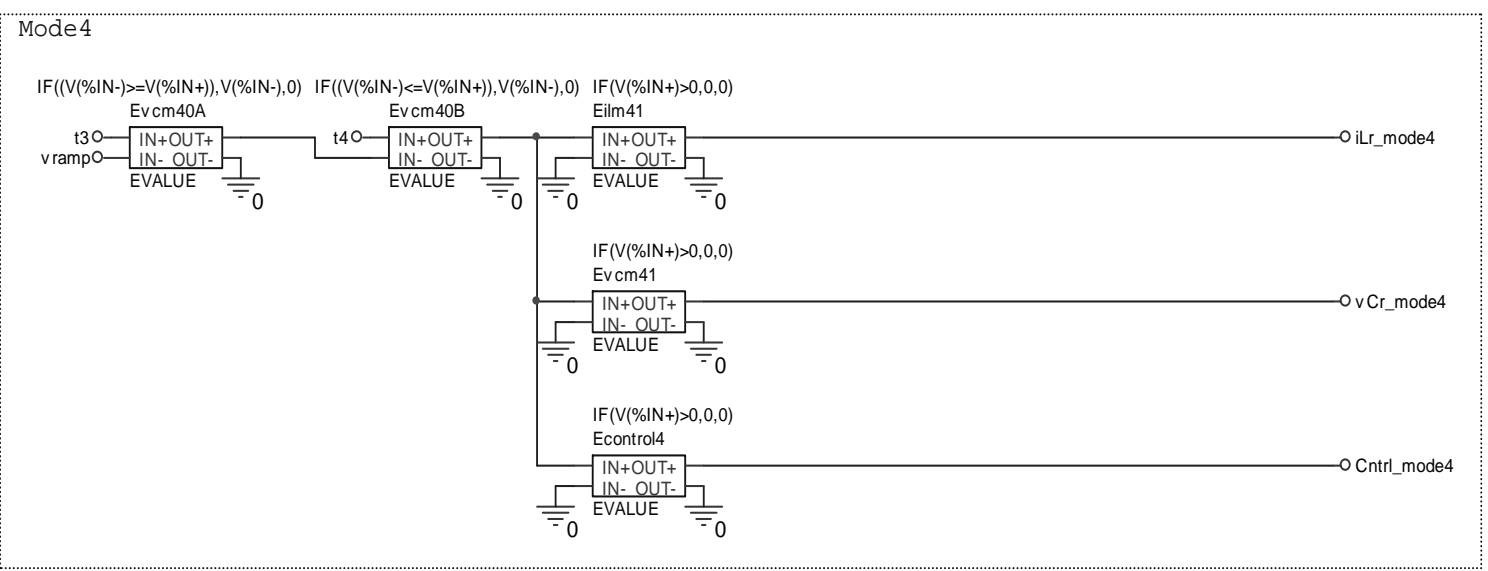

Figure 3.6: MODE4 Pspice block

The last stage in this block is summing the outputs from different mode subblocks to generate the steady-state $I_{L r}$ and $V_{C r}$ waveform. Figure 3.7 shows the Pspice summation block. 


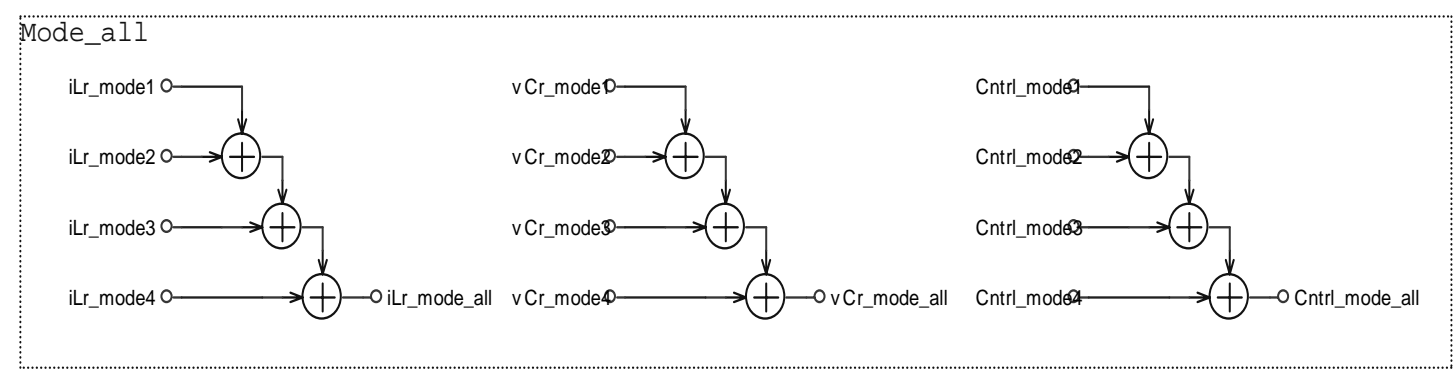

Figure 3.7: All Modes summation Pspice block

Another important function in the $D R W$ block is a sub-block that finds the different time intervals $\left(t_{1}, t_{2}, t_{3}, t_{4}\right)$ from $(\alpha, \beta, \gamma, \sigma)$ values. The block is based on simple equations summarized below and Figure 3.8 shows Pspice implementation.

$$
\begin{gathered}
t_{1}=\frac{\alpha}{w_{o}} \\
t_{2}=\frac{\beta}{w_{o}}+t_{1} \\
t_{3}=\frac{\gamma}{w_{o}}+t_{2} \\
t_{4}=\frac{\sigma}{w_{o}}+t_{3}
\end{gathered}
$$




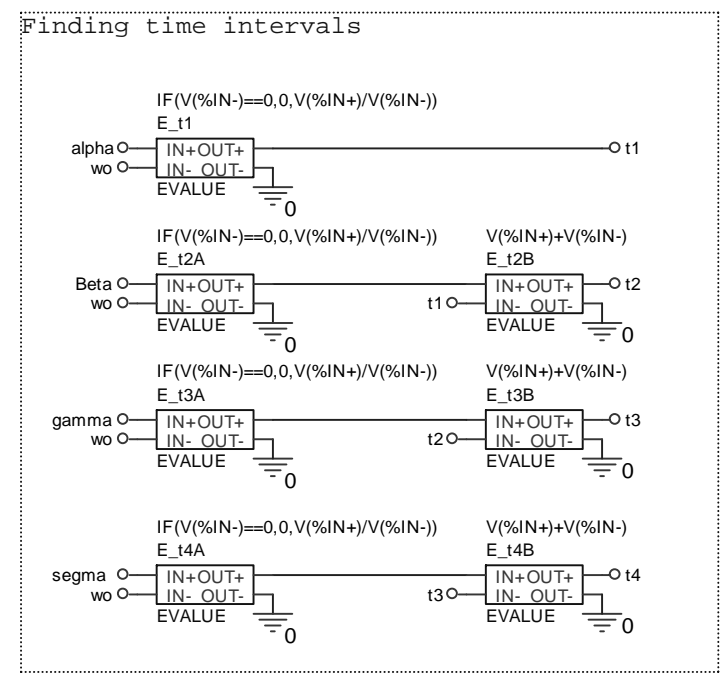

Figure 3.8: Finding time intervals Pspice block

The $D R W$ block was tested using soft-switching cell inductance $L_{r}=3 \mu \mathrm{H}$, softswitching cell capacitance $C_{r}=0.02 \mu \mathrm{F}$, switching frequency $f_{s}=250 \mathrm{KHz}$, input voltage $V_{\text {in }}=25 \mathrm{~V}$ and load $R_{o}=12 \Omega$. Figure 3.9 shows the simulation results.

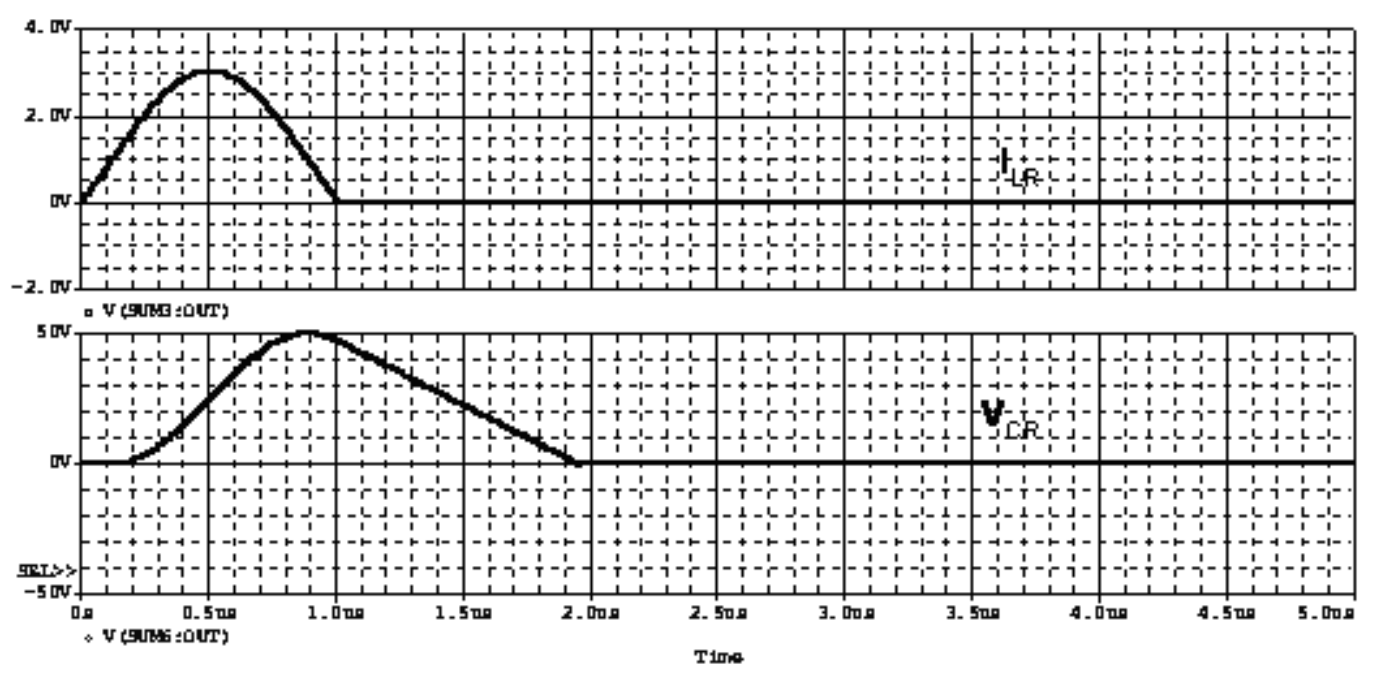

Figure 3.9: $I_{L r}$ and $V_{C r}$ steady-state waveform 


\subsection{Generating the State-Plane Diagram}

Using the resonant inductor current and the resonant capacitor voltage from the model outputs, the state-plane diagram can be generated. The State-plane diagram is a two dimensional graph that sketches the state variables for a given switching cell at different modes of operation. It is helpful in gaining better understanding of the cell operation and is used in determining the control characteristic of soft-switching cell. In other words, the converter design gain as a function of frequency and load can be obtained from the state-plane diagram. Moreover, the state variables peak values can be determined from the state plane. Figure 3.10 shows the generalized state plane diagram for ZCS-QRC Switching Cell [28-32].

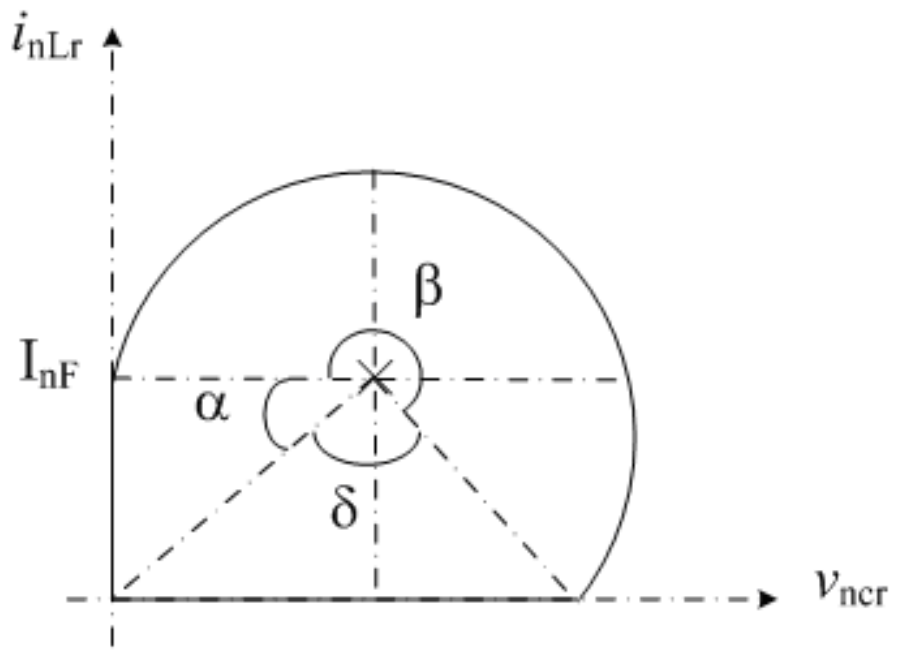

Figure 3.10: The Generalized State plane for ZCS-QRC Switching Cell 
The Generalized state plane diagram analysis can be used to derive other characteristic equations for the generalized switching cell such as the generalized peak resonant capacitor voltage $V_{n c r, P}$, the generalized peak resonant inductor current $I_{n L r, P}$, the generalized peak switch current and voltage $I_{n, s w-p} \& V_{n, s w-p}$ and generalized average switch current and voltage $V_{n, s w-a v e} \& I_{n, s w-a v e}$. The equations for the above parameters are summarized below $[3,30,31]$ :

$$
\begin{gathered}
V_{n c r, P}=2 V_{n g} \\
I_{n L r, P}=I_{n F}+\frac{Q V_{n g}}{M} \\
I_{n, s w-p}=I_{n F}+V_{n g} \\
V_{n, s w-p}=V_{n g} \\
I_{n, s w-a v e}=I_{n F}-I_{n D}=I_{n F}-I_{n b} \\
V_{n, s w-a v e}=V_{n g}-V_{n F}
\end{gathered}
$$

The state plane figure can be generated in the unified model by using $I_{L r}$ and $V_{C r}$ steady-state waveform block " $D R W$ Block." The $D R W$ Block generates the steady-state waveforms for the soft-switching cell inductor current $I_{L r}$ and the soft-switching cell capacitor voltage $V_{C r}$. The state plane diagram can be generated by plotting $V_{C r}$ versus $I_{L r}$ in Pspice. 
The state-plane block was tested for ZCS-QRC Buck using soft-switching cell inductance $L_{r}=3 \mu \mathrm{H}$, soft-switching cell capacitance $C_{r}=0.02 \mu \mathrm{F}$, switching frequency $f_{s}=250 \mathrm{KHz}$, input voltage $V_{\text {in }}=25 \mathrm{~V}, V_{\text {out }}=12 \mathrm{~V}$ and load $R_{o}=12 \Omega$. The corresponding theoretical values are calculated as follows:

First the voltage gain is:

$$
M=\frac{V_{o}}{V_{\text {in }}}=\frac{12}{25}=0.48
$$

Choosing $f_{n s}=0.4$ and using the control characteristic curve Section 1.7 , this results in $Q=1$. The characteristic impedance can be found by:

$$
Z_{o}=\frac{R_{o}}{Q}=\sqrt{\frac{L}{C}}=12.0 \Omega
$$

The normalized switching frequency and the angular frequency can be found by:

$$
\begin{aligned}
& f_{o}=\frac{f_{s}}{f_{n s}}=\frac{250 e 3}{0.4}=625 \mathrm{kHz} \\
& w_{o}=2 \pi f_{o}=3.9270 \mathrm{e}+6
\end{aligned}
$$

The peak inductor current can be calculated by:

$$
I_{L-P e a k}=I_{o}+\frac{V_{\text {in }}}{Z_{o}}=3 \mathrm{~A}
$$

And the peak capacitor voltage is:

$$
V_{C-P e a k}=2 V_{\text {in }}=50 \mathrm{~V}
$$


And finally time intervals can be calculated from the following expressions:

$$
\begin{gathered}
\alpha=\frac{M}{Q}=0.480 \mathrm{rad} \\
\beta=\operatorname{SIN}^{-1}\left(\frac{-M}{Q}\right)=\operatorname{SIN}^{-1}\left(\frac{-0.48}{1}\right)=3.6422 \mathrm{rad} \\
\gamma=\frac{Q}{M}(1-\operatorname{COS} \beta)=\left(\frac{1}{0.48}\right)(1-\operatorname{COS}(3.6422))=3.911 \mathrm{rad}
\end{gathered}
$$

Figure 3.11 shows the simulation results and Table 3.2 shows a comparison between theoretical values and model calculated values.

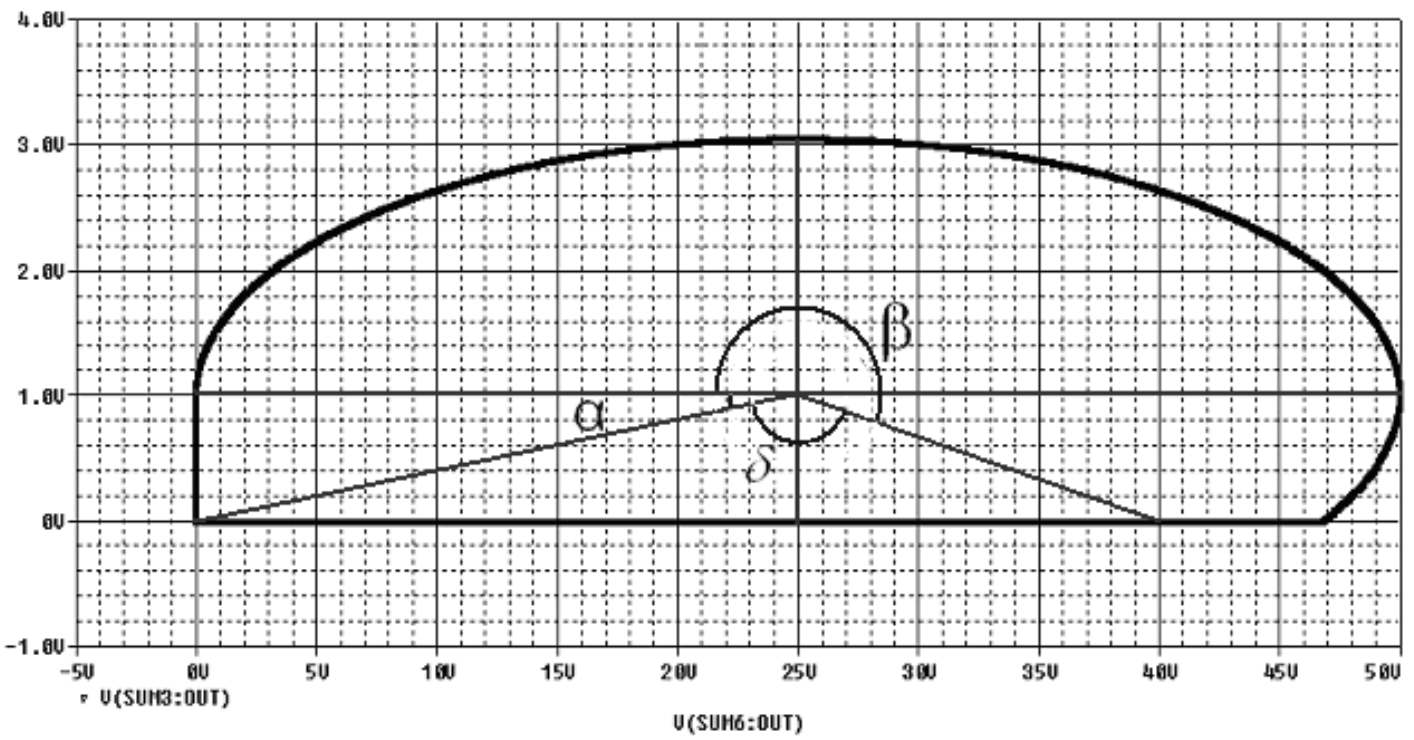

Figure 3.11: Switching cell state-plane diagram 
Table 3.2: Comparison between theoretical values and Model calculated values.

\begin{tabular}{|c|c|c|}
\hline Parameter & Theoretical Value & Model Value \\
\hline$\alpha$ & $0.480 \mathrm{rad}$ & $0.4898 \mathrm{rad}$ \\
\hline$\beta$ & $3.6422 \mathrm{rad}$ & $3.6535 \mathrm{rad}$ \\
\hline$\gamma$ & $3.911 \mathrm{rad}$ & $3.8207 \mathrm{rad}$ \\
\hline$\sigma$ & $7.6748 \mathrm{rad}$ & $7.744 \mathrm{rad}$ \\
\hline$I_{L-\text { Peak }}$ & $3 \mathrm{~A}$ & $3 \mathrm{~A}$ \\
\hline$V_{\text {C-Peak }}$ & $50 \mathrm{~V}$ & $50 \mathrm{~V}$ \\
\hline
\end{tabular}

\subsection{Feedback Design}

The unified steady-state model can be used for feedback design in applications where the output voltage is regulated by changing the switching frequency $f_{s}$.Figure 3.12 shows a general block diagram of the closed loop system using Pspice unified model. It should be noted here that feedback design using the unified model is still in progress and this section presents only the work done so far. Future publications will contain a detailed complete discussion about feedback design using the unified model.

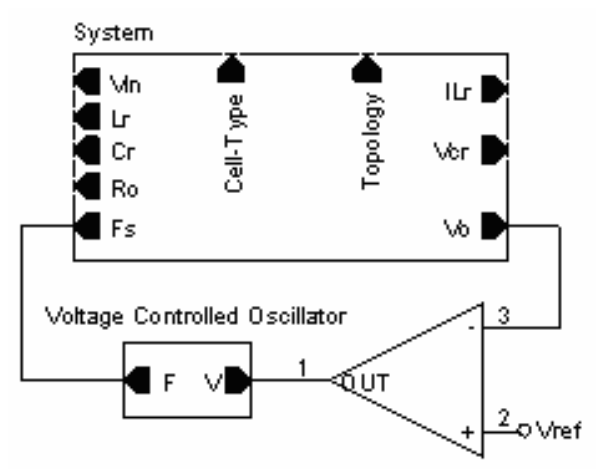

Figure 3.12: Closed loop using the unified general model 
The closed loop starts by sensing the output voltage from the unified model; this voltage is then compared with the desired reference voltage. The error signal generated is fed to a voltage-controlled oscillator which in turn generates a frequency value that depends on the error signal. This new frequency will regulate the output voltage.

The unified model in its current structure cannot regulate the output voltage since the model cannot detect frequency changes. The next sections describe the necessary changes in SOL block to detect frequency changes and to find new solutions if there is a frequency change.

\subsubsection{Detecting changes in switching frequency}

For Quasi resonant cells the output voltage is regulated by changing the switching frequency. The unified model needs to detect frequency changes to update its different parameters and its final steady-state gain solution. There are two difficulties in building this functionality: first, building the circuit that detects changes in the input variable; second, the unified model at this stage needs $1 \mathrm{u}$ second to find a steady-state solution. The detecting circuit should not interrupt the unified model blocks within the $1 \mathrm{u}$ second period and at the same time it should latch a signal to the solution block showing that a change has occurred in the switching frequency so that a new solution cycle starts.

Frequency change detection circuit was built using the sample and hold concept. The switching frequency $f_{s}$ is processed by two sample and hold circuits. A sample and hold circuit is simply a switch and a capacitor-the switch turns ON and the capacitor starts charging to the input voltage value, and then the switch turns OFF and the capacitor 
holds the last stored value until the switch turns ON again. One of the two "sample and Hold" circuits is delayed by $1 \mathrm{u}$ second from the other circuit and both circuits have a sampling period of $2 \mathrm{u}$ seconds. In this way, the frequency detection circuit updates the solving block of new changes at the correct time without interrupting the solving process. The frequency change detection circuit is shown in Figure 3.13.

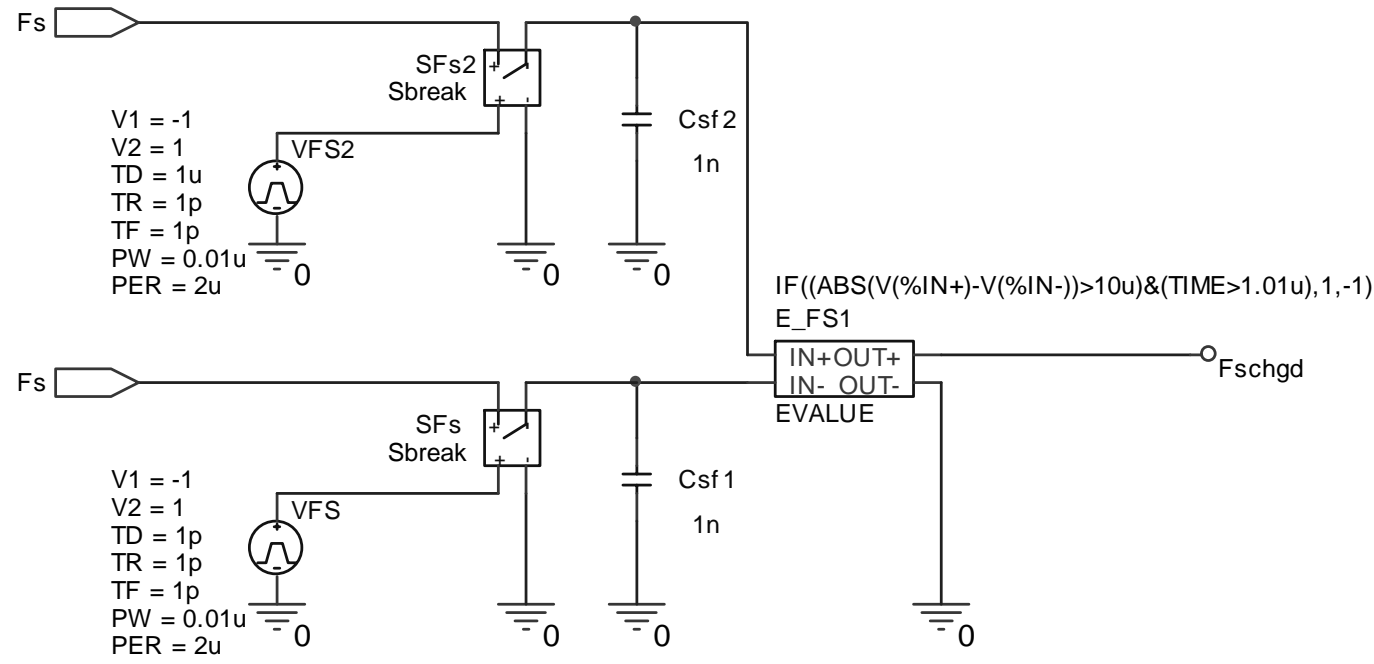

Figure 3.13: Frequency change detection circuit

The frequency change detection circuit was tested with a hypothetical change in the switching frequency shown in Figure 3.14. Figure 3.15 shows the sampling pulses for "Sample and Hold" Circuits 1 and 2. Figure 3.16 shows the sampled waveform and Finally Figure 3.17 shows the output of frequency change detection block. 


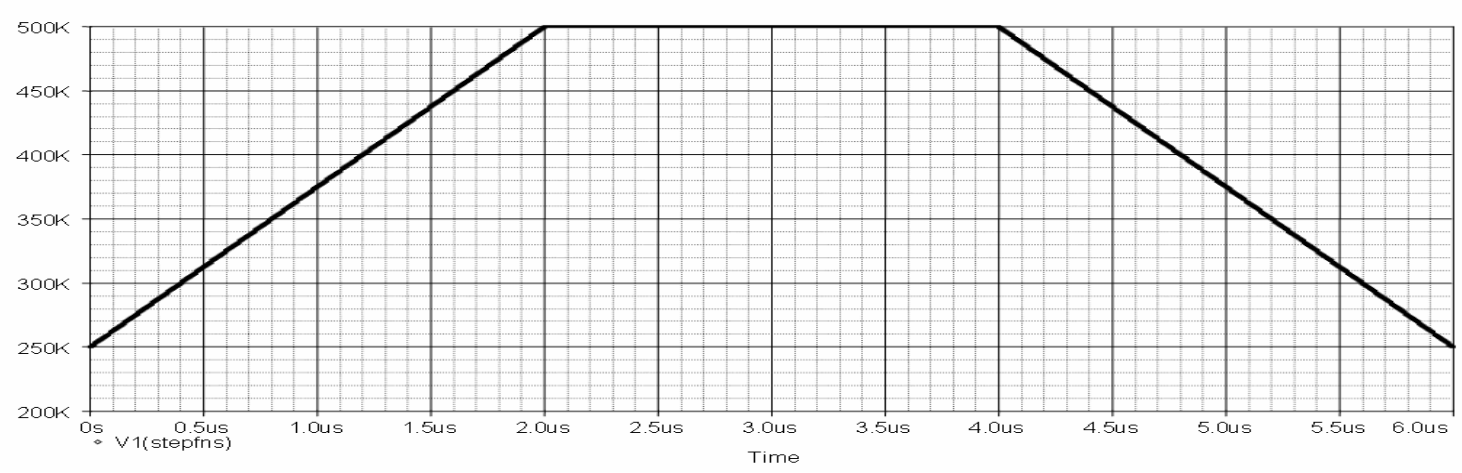

Figure 3.14: Frequency change test signal

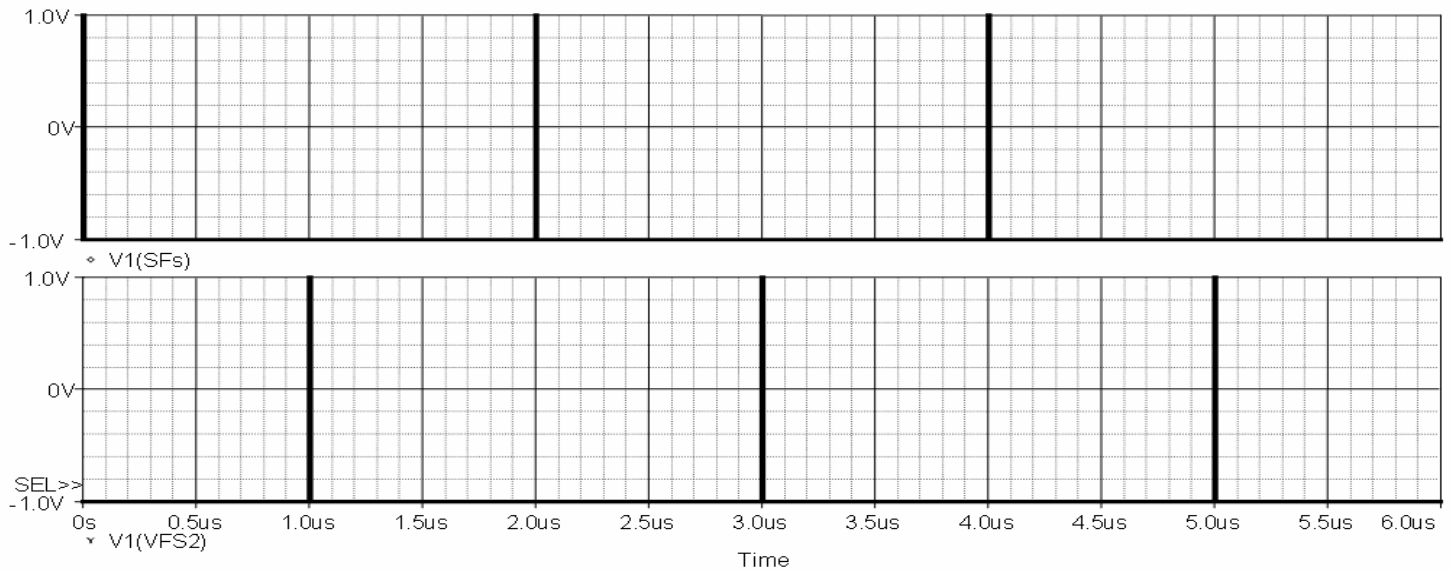

Figure 3.15: Sampling Pulses

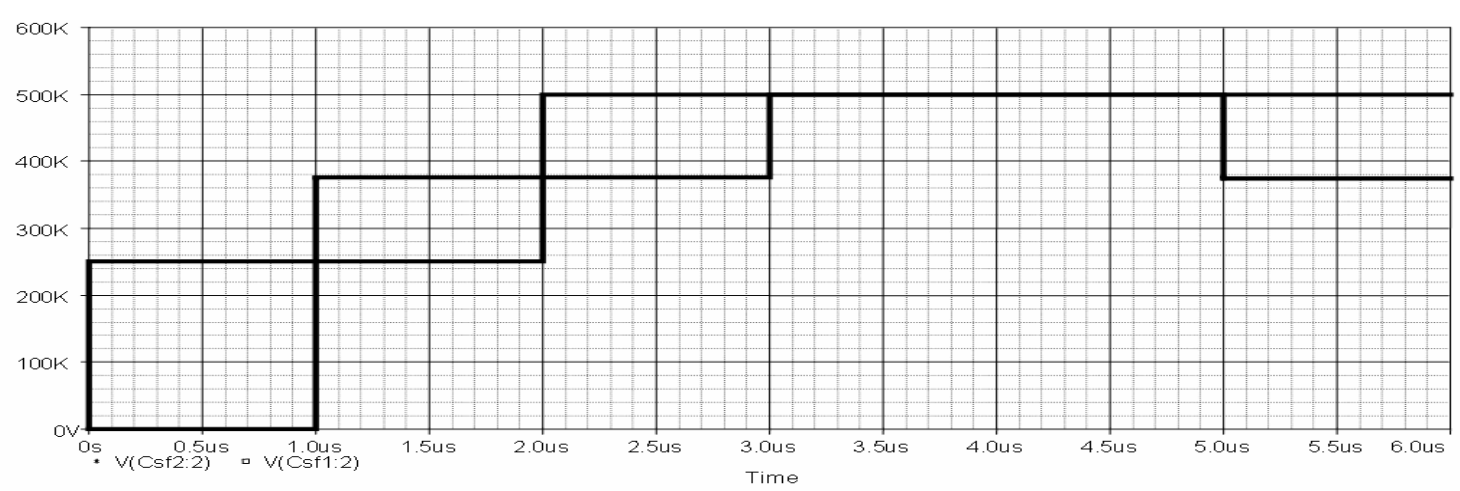

Figure 3.16: Sampled Test Waveform 


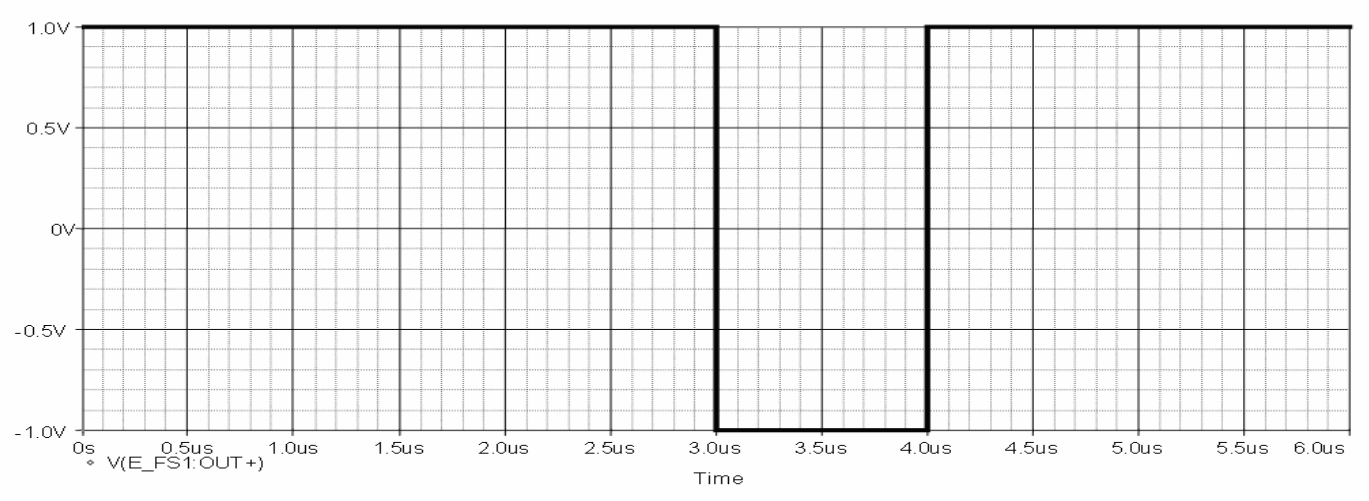

Figure 3.17 Frequency change detection block output result

\subsubsection{Modified SOL block}

A major problem with the OR gate solution introduced in Chapter 2 (2.6.6.1) is that once the OR gate output is logic 1, it will be always at logic 1 regardless of input changes, so if the switching frequency changes for some reason and a new steady-state solution is needed, the circuit cannot process the new solution since the output switch will be OFF, and the output capacitor will not change to any new value. One idea to make the SOL block see frequency changes is to use a counter; the counter is set with an initial value. When the error signal goes below the threshold, the counter value is incremented and another $\mathrm{ABM}$ monitors the counter value. Whenever the counter value changes, this ABM stops the solution capacitor from charging by turning the solution Switch OFF.

A Modified version of the counter idea is to use a capacitor as a storage element. In other words, when the error goes below the specified threshold, the capacitor is charged with new values. Another ABM monitors the capacitor voltage; whenever it increases, it generates a control signal to keep the output switch OFF. Figure 3.18 shows the circuit implementation. 


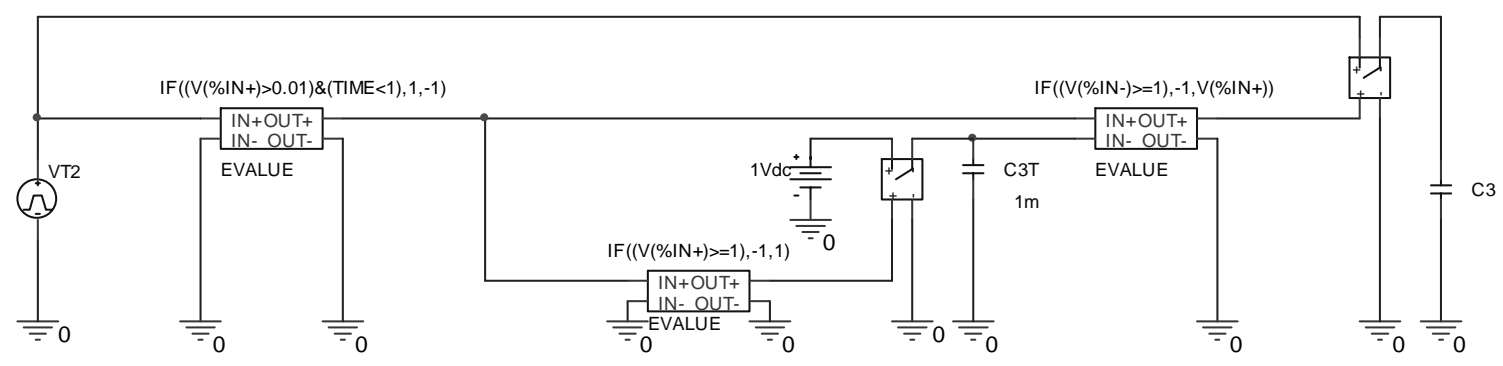

Figure 3.18: Pspice implementation of counter idea

The next stage is modifying the control circuit to see changes in switching frequency $f_{s}$. The circuit that detects frequency changes was explained in Section 3.4.1; this circuit generates $+1 \mathrm{v}$ when there is a frequency change and generates $-1 \mathrm{v}$ when the frequency keeps constant. The circuit output is generated every $1 \mathrm{u}$ second.

Frequency changes mean a new solution is needed. A modified version of the capacitor-counter circuit uses two sub-circuits that control the counter-capacitor voltage, in other words, the first counter-capacitor sub-circuit monitors the error value; if the error goes below a certain threshold it charges the counter-capacitor to a new voltage. Another $\mathrm{ABM}$ monitors the counter-capacitor voltage, and if the voltage exceeds a certain limit, the ABM will turn the solution switch OFF, and the solution capacitor will hold the latest charged value. The second counter-capacitor sub-circuit monitors the output of frequency change detection circuit (Section 3.4.1). If there is a frequency change it will discharge the counter-capacitor to a lower voltage so the monitoring $\mathrm{ABM}$ will close the solution ON again and the solution capacitor will charge to a new value. The modified capacitorcounter circuit is shown in Figure 3.19. 


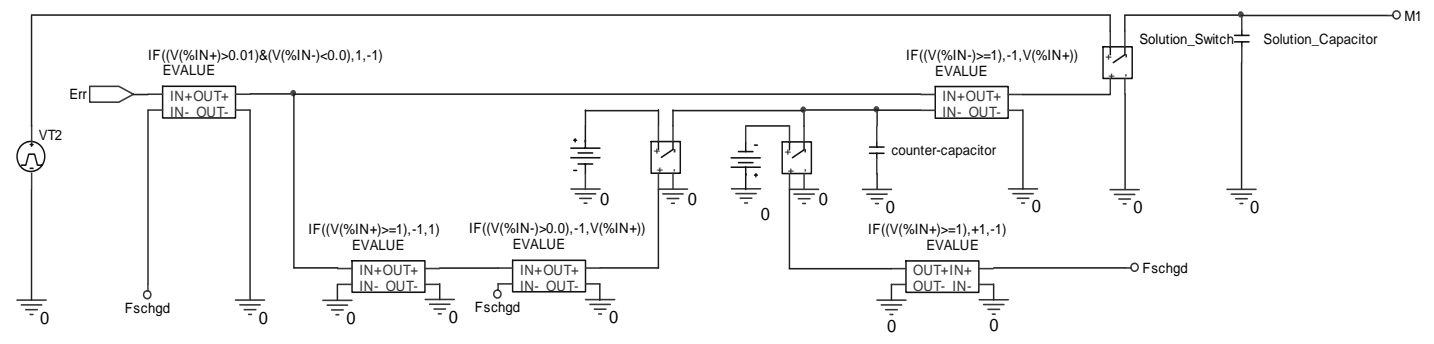

Figure 3.19 modified capacitor-counter circuit

To test the modified solution block, the system was simulated using ZCS-QRC Buck with soft-switching cell inductance $L_{r}=3 \mu \mathrm{H}$, soft-switching cell capacitance $C_{r}=0.02 \mu \mathrm{F}$, input voltage $V_{\text {in }}=25 \mathrm{~V}, V_{\text {out }}=12 \mathrm{~V}$, load $R_{o}=12 \Omega$. Switching frequency $f_{s}$ that step from $f_{s}=500 \mathrm{KHz}$ to $f_{s}=250 \mathrm{KHz}$. Figure 3.20 shows the unified model step response.

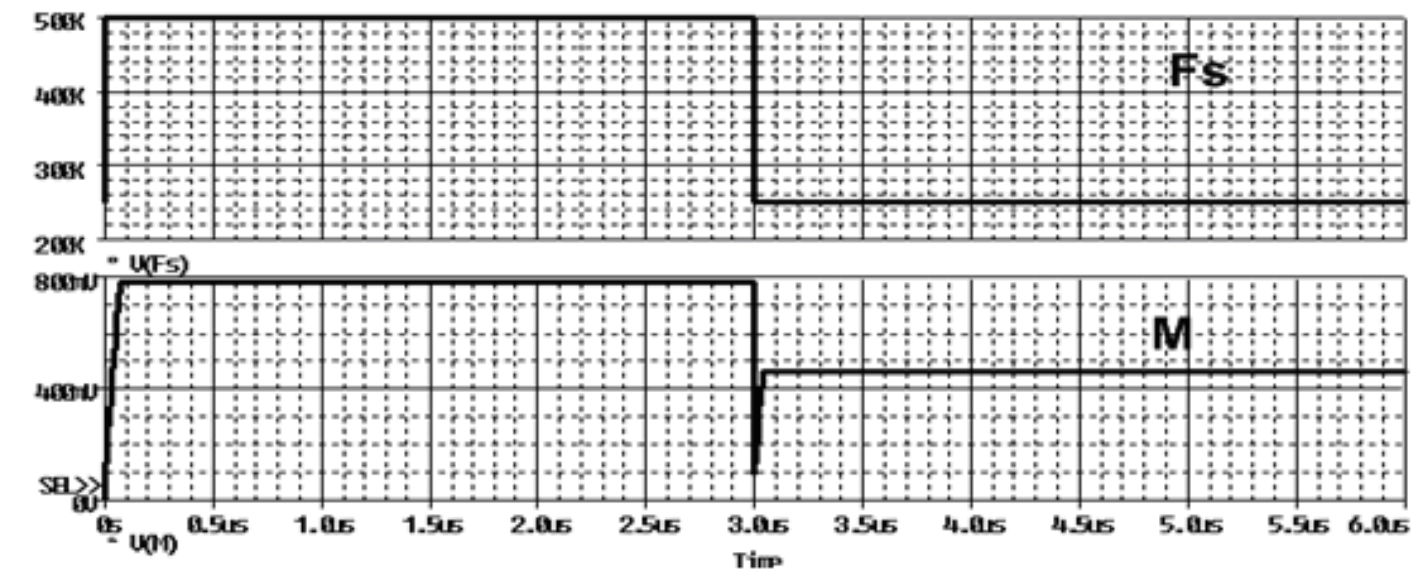

Figure 3.20 Unified Model step response 


\subsection{Unified Model for common DC-DC converters families}

The unified steady-state simulation model can be used with the most common DC-DC converter family, namely, buck, boost, buck-boost, cuck, zeta and sepic. The user chooses the soft-switching cell type and connects the Unified model in the desired topology configuration. In order for the unified model to generate the correct steady-state values, the model needs to know the topology it is connected to. In previous chapters, the unified model was used in one topology type, the buck converter. This section summarizes how the unified model can be used with other DC-DC converters families.

\subsubsection{Unified Model for Boost converter family}

For the Boost topology and based on the discussion on chapter 1, the ZCS-QRC will have the same switching waveforms and the same modes of operation regardless of the cell orientation $[2,3]$. Using the generalized steady-state analysis in section 1.6 , the generalized interval equations are:

$$
\begin{gathered}
\alpha=\omega_{o}\left(t_{1}-t_{0}\right)=\frac{M I_{n F}}{Q V_{n g}} \\
\beta=\omega_{o}\left(t_{2}-t_{1}\right)=\sin ^{-1}\left(-\frac{M I_{n F}}{Q V_{n g}}\right) \\
\gamma=\omega_{o}\left(t_{3}-t_{2}\right)=\frac{Q V_{n g}}{M I_{n F}}(1-\cos \beta) \\
\delta=\omega_{o}\left(\left(t_{0}+T_{s}\right)-t_{3}\right)=\frac{2 \pi}{f_{n s}}-\alpha-\beta-\gamma
\end{gathered}
$$


By substituting for the generalized parameters $\left(V_{n g}\right.$ and $\left.I_{n F}\right)$ from Table 1.1 the corresponding intervals for the Boost topology are:

$$
\begin{gathered}
\alpha=\omega_{o}\left(t_{1}-t_{0}\right)=\frac{M}{Q} \\
\beta=\omega_{o}\left(t_{2}-t_{1}\right)=\sin ^{-1}\left(-\frac{M}{Q}\right) \\
\gamma=\omega_{o}\left(t_{3}-t_{2}\right)=\frac{Q}{M}(1-\cos \beta) \\
\delta=\omega_{o}\left(\left(t_{0}+T_{s}\right)-t_{3}\right)=\frac{2 \pi}{f_{n s}}-\alpha-\beta-\gamma
\end{gathered}
$$

It can be noted that the generalized interval values for the Boost topology are exactly the same as the generalized interval values for the Buck topology, and this means the corresponding Pspice blocks will be the same. Also using the generalized steady-state analysis in section 1.6 , the generalized gain equation is:

$$
V_{n b c}=\frac{f_{n s}}{2 \pi}\left[\frac{M I_{n F}}{2 Q} \gamma^{2}-V_{n g}(\beta+\gamma-\sin \beta-\gamma \cos \beta)\right]
$$

By substituting for the generalized parameters $\left(V_{n F}, V_{n g}\right.$, and $\left.I_{n F}\right)$ from Table 1.1 in Equation (1.17), the gain equation for the Boost converter is: 


$$
(1-M)=\frac{M f_{n s}}{2 \pi}\left[\frac{M}{2 Q} \gamma^{2}-(\beta+\gamma-\sin \beta-\gamma \cos \beta)\right]
$$

Rearrange the equation:

$$
f_{n s}=\frac{2 \pi\left(\frac{1-M}{M}\right)}{\frac{M}{2 Q} \gamma^{2}-(\beta+\gamma-\sin \beta-\gamma \cos \beta)}
$$

The Pspice implementation of ZCS-QRC Boost is exactly the same as the ZCSQRC Buck. From the equation derived above, the generalized interval equations are the same as the Buck converter, and the same developed Pspice blocks can be used. For the generalized gain equation, the only difference is in the multiplication factor $-\left(\frac{1-M}{M}\right)$.

Figure 3.21 the Pspice implementation for the Error block for the Boost topology.

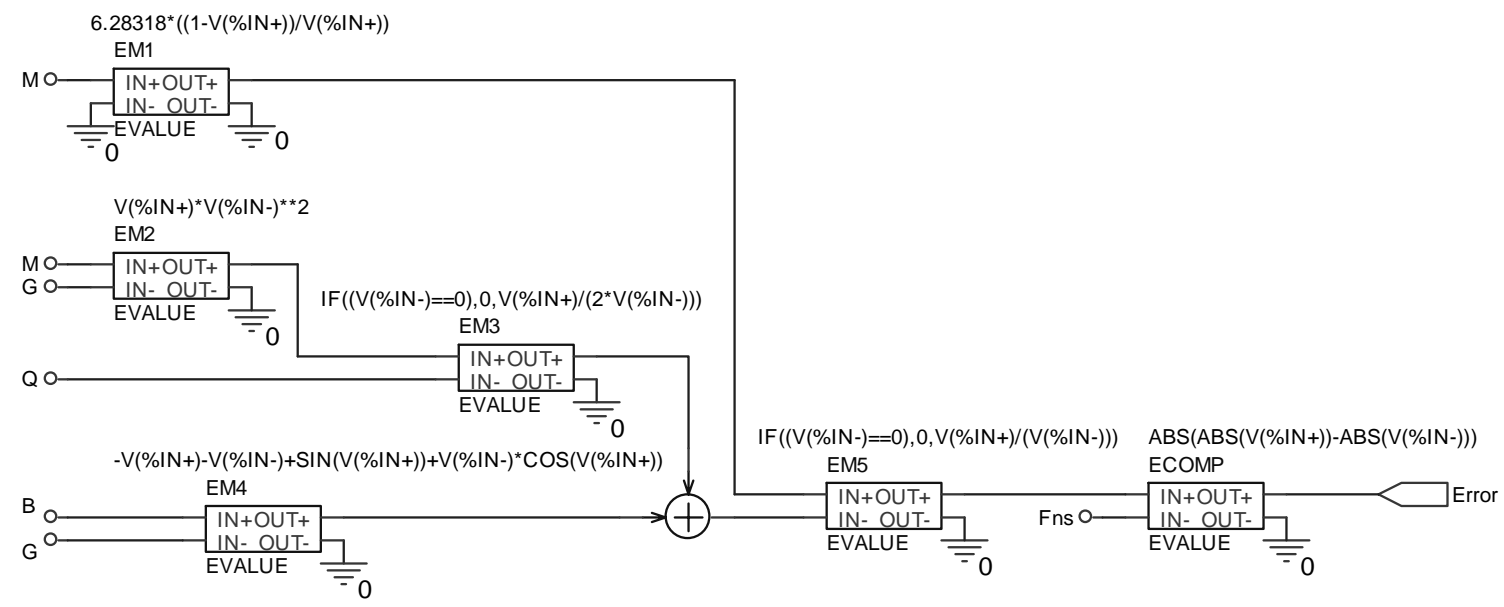

Figure 3.21 Error block for the Boost topology 


\subsubsection{Unified Model for Buck-Boost converter family}

For the Buck-Boost topology and based on the discussion on Chapter 1, the ZCSQRC will have the same switching waveforms and same modes of operations regardless of cell orientation $[2,3]$. Using the generalized steady-state analysis in section 1.6 , the generalized interval equations are:

$$
\begin{gathered}
\alpha=\omega_{o}\left(t_{1}-t_{0}\right)=\frac{M I_{n F}}{Q V_{n g}} \\
\beta=\omega_{o}\left(t_{2}-t_{1}\right)=\sin ^{-1}\left(-\frac{M I_{n F}}{Q V_{n g}}\right) \\
\gamma=\omega_{o}\left(t_{3}-t_{2}\right)=\frac{Q V_{n g}}{M I_{n F}}(1-\cos \beta) \\
\delta=\omega_{o}\left(\left(t_{0}+T_{s}\right)-t_{3}\right)=\frac{2 \pi}{f_{n s}}-\alpha-\beta-\gamma
\end{gathered}
$$

By substituting for the generalized parameters $\left(V_{n g}\right.$ and $\left.I_{n F}\right)$ from Table 1.1, the corresponding intervals for the Buck-Boost topology are:

$$
\begin{gathered}
\alpha=\omega_{o}\left(t_{1}-t_{0}\right)=\frac{M}{Q} \\
\beta=\omega_{o}\left(t_{2}-t_{1}\right)=\sin ^{-1}\left(-\frac{M}{Q}\right) \\
\gamma=\omega_{o}\left(t_{3}-t_{2}\right)=\frac{Q}{M}(1-\cos \beta) \\
\delta=\omega_{o}\left(\left(t_{0}+T_{s}\right)-t_{3}\right)=\frac{2 \pi}{f_{n s}}-\alpha-\beta-\gamma
\end{gathered}
$$


It can be noted that the generalized interval values for the Buck-Boost topology are exactly the same as the generalized interval values for the Buck topology and this means the corresponding Pspice blocks will be the same. Using also the generalized steady-state analysis in section 1.6, the generalized gain equation is:

$$
V_{n b c}=\frac{f_{n n}}{2 \pi}\left[\frac{M I_{n F}}{2 Q} \gamma^{2}-V_{n g}(\beta+\gamma-\sin \beta-\gamma \cos \beta)\right]
$$

By substituting for the generalized parameters $\left(V_{n F}, V_{n g}\right.$, and $\left.I_{n F}\right)$ from Table 1.1 in Equation (1.17), the gain equation for the Buck-Boost converter is:

$$
-M=\frac{(1+M) f_{n s}}{2 \pi}\left[\frac{M}{2 Q} \gamma^{2}-(\beta+\gamma-\sin \beta-\gamma \cos \beta)\right]
$$

Rearrange the equation:

$$
f_{n s}=\frac{-2 \pi\left(\frac{M}{1+M}\right)}{\frac{M}{2 Q} \gamma^{2}-(\beta+\gamma-\sin \beta-\gamma \cos \beta)}
$$

The Pspice implementation of ZCS-QRC Buck-Boost is exactly the same as the ZCS-QRC Buck. From the equation derived above the generalized interval equations are the same as the Buck converter and the same developed Pspice blocks can be used. For 
the generalized gain equation, the only difference is in the multiplication factor $\left(\frac{M}{1+M}\right)$.

Figure 3.22 the Pspice implementation for the Error block for the Buck-Boost topology.

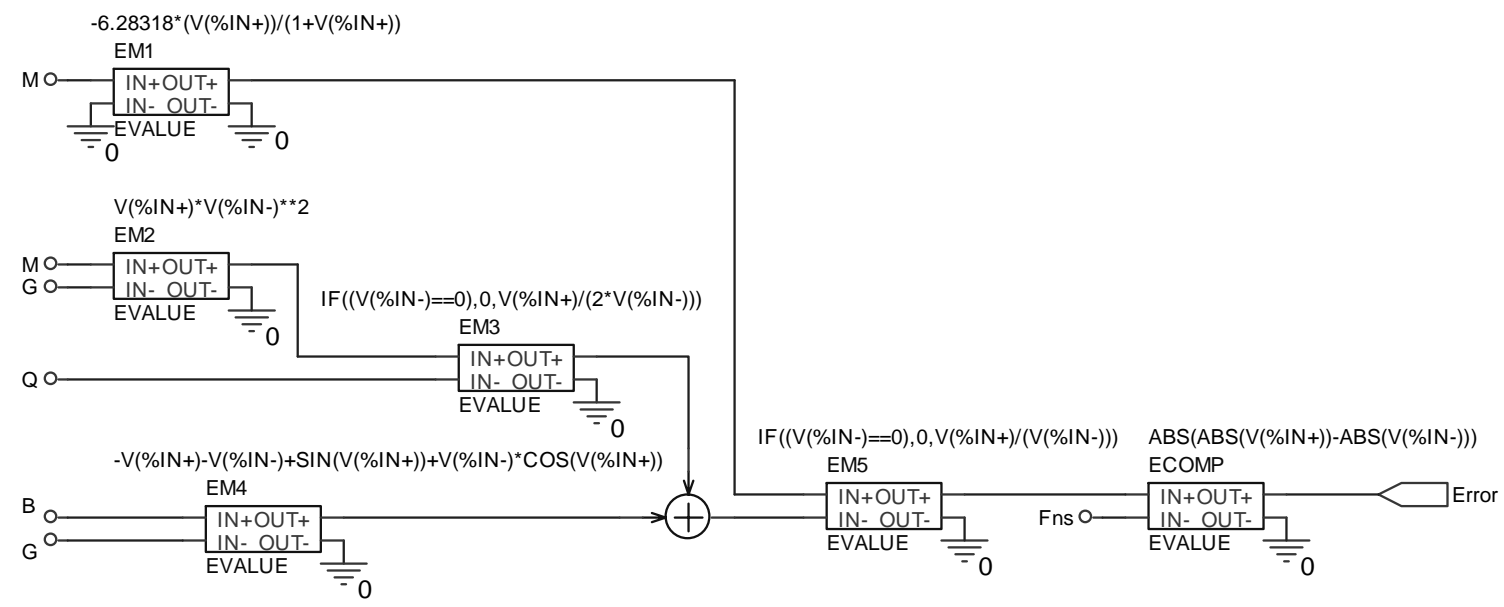

Figure 3.22 Error block for the Buck-Boost topology

The other DC-DC converter topologies, the cuck, zeta and sepic, will have the same generalized interval equations and the same steady-state gain equation as the BuckBoost topology (Equation 3.15). For this reason, they will have the same Pspice implementation shown in Figure 3.22.

\subsubsection{Selecting between different topologies}

In order for the unified model to calculate the correct steady-state values and to generate the correct switching waveforms, the model needs to know the topology it is connected to. The unified model has a "Topology" pin input through which the user selects the topology type i.e. Buck, Boost, Buck-Boost. Figure 3.23 show the complete Error block with topology selection feature. 


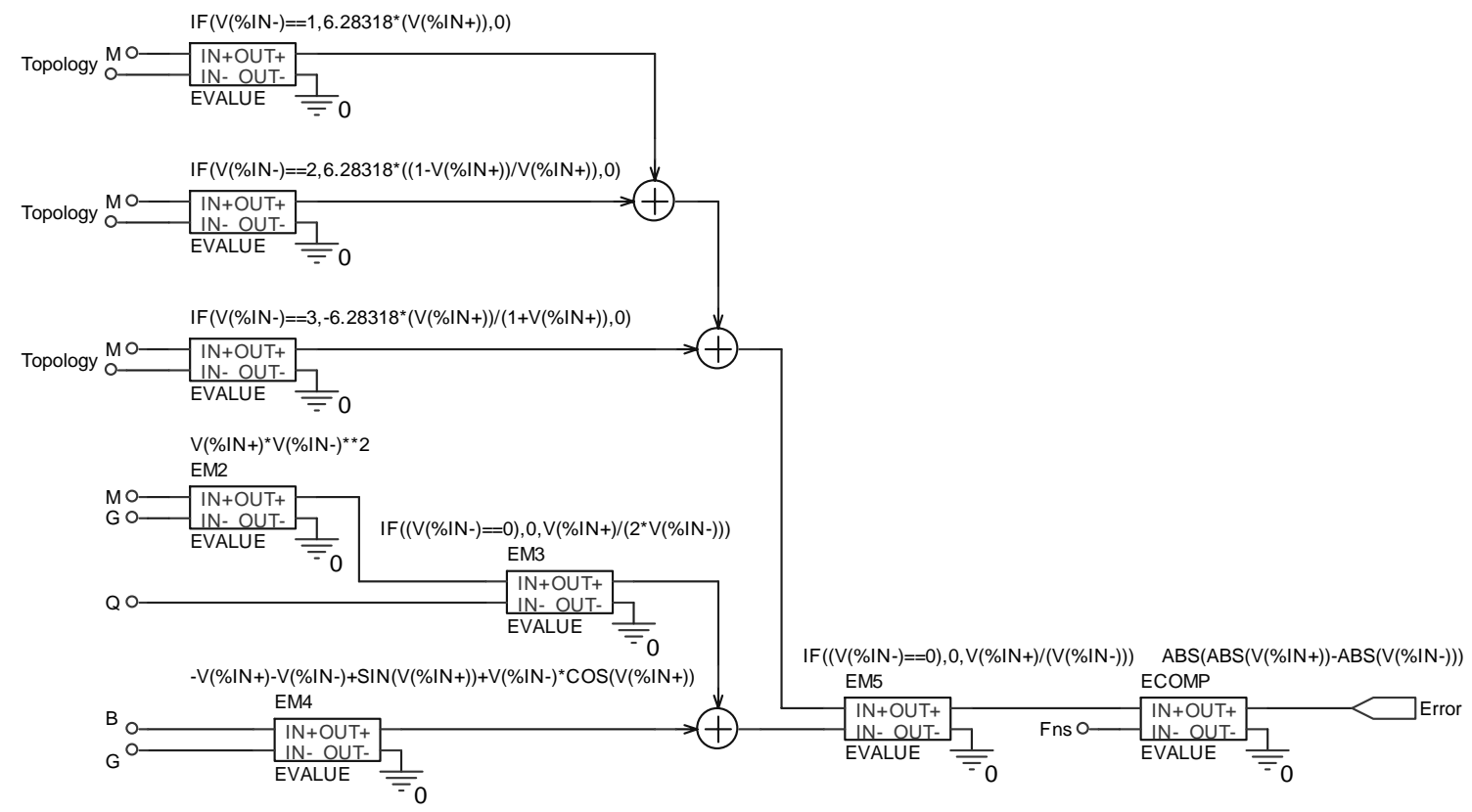

Figure 3.23 Complete Error block with topology selection

From figure 3.23, the Topology pin corresponds to a voltage level, the error block was programmed to solve buck equations when topology pin equals 1 volt, and to solve boost equations when topology pin equals 2 volt and finally to solve buck-boost equations when topology pin equals 3 volt. When a certain topology is selected its corresponding equation will be solved and all other equations outputs will be equated to zero finally the block adds all the outputs to get the final solution. 


\section{CHAPTER 4}

\section{CONCLUSION}

\subsection{Summary}

This thesis presents a unified steady-state model for soft-switching converters; the unified model is an easy and versatile way to simulate soft-switching and other complicated topologies. Also, the unified model provides designers with a better understanding of soft-switching topologies and offers a fast steady-state solution in addition to providing accurate waveforms for different cell parameters and state-plane diagrams. It is also a good educational tool.

The unified model is based on generalized analysis which is a new technique to analyze and to find steady-state solutions for soft-switching cells. Generalized analysis is based on the idea that soft-switching cells will have the same modes of operation and the same switching waveforms in spite of cell orientation. So instead of analyzing switching cells contained in different converters as a one topology, only the switching cell needs to be analyzed and the result can be applied to different DC-DC converter families using transformation tables. To introduce unified model development issues and challenges, the zero current switching (ZCS) quasi-resonant (QRC) unified switching cell was selected.

The proposed unified simulation model is implemented in Pspice/Orcad $\AA$ simulation software to provide a model that can be used as a part of complete simulation schematic that may include other models in Pspice/Orcad®. Using the mathematical equations approach to implement the model requires the ability to implement 
programming loops and to perform instructions in a certain sequence, which was the main challenge in this work because Pspice/Orcad $₫$ is not a programming language and soft switching cells have complicated set of equations that describe the operation modes.

The unified steady-state model is useful in soft-switching cells analysis and design; the user connects the simulation model to the desired topology configuration and the Model calculates the steady-state gain solution for the user. The model also provides the user with different necessary plots needed for soft-switching cell design. This includes the internal switching cell information such as switching cell inductor current $I_{L r}$ and the switching cell capacitor voltage $V_{C r}$, and their corresponding state-plane diagram. The model can also be used in feed-back design applications where the output voltage is regulated by changing the switching frequency.

\subsection{Future work}

Future work will focus on optimizing the unified model accuracy and speed. The model speed can be increased by implementing fast numerical techniques to solve the unified equation non-linear equation. Model accuracy can be increased by searching for the error signal minimum point instead of using a threshold value. Feed-back design using the unified model is still in its early stages. It needs more research and work. 


\section{REFERENCES}

[1] I. Batarseh, "Power Electronics Circuits," John Wiley, Fall 2000.

[2] J. Abu-Qahouq, "Generalized Analysis of Soft-Switching DC-DC Converters Families," Master Thesis, University of Central Florida,2000.

[3] J. Abu-Qahouq, I. Batarseh, "Unified steady-state analysis of soft-switching DC-DC converters," IEEE Trans. Power Electron. vol. 17, pp. 684 - 691, Sept. 2002.

[4] G. Hua, "Soft-Switching Techniques for Pulse-Width-Modulated Converters," Virginia Power Electronics Center, 1994.

[5] G. Hua, F.C. Lee, "An overview of soft-switching techniques for PWM converters," EPE Journal, vol. 3, no. 1, March 1993.

[6] G. Hua, F.C. Lee, "Soft-switching techniques for PWM converters," International Conf. on Industrial Electronics, Control and Instrumentation, 1993.

[7] G. Hua and F. Lee "Evaluation of Switched-Mode Power conversion Topologies," The first international Power Electronics and Motion Control Conf. (IPEMC), Vol.1, pp.12-26,1994.

[8] E.J. Miller "Resonant Switching Power Conversion," IEEE Power Electronics Specialists conference Rec. pp. 206-211, 1976.

[9] K. Liu, R. Oruganti, and F. C. Lee, "Resonant Switches - Topologies and Characteristics," IEEE Trans. On Power Electronics, Vol. PE-2, No.1, pp. 62-74, 1987.

[10] T. Mizoguchi, T. Ohgai, and T. Ninomiya, "A Family of Single-Switch ZVS-CV DC-DC Converters," IEEE Applied Power Electronics Specialists Conf. Vol. 2, pp. 1392-1398,1994.

[11] C.P. Henze, H.C. Martin, D.W. Parsley, "Zero-voltage-switching in high frequency power converters using pulse width modulation," IEEE Applied Power Electronics Conf. Proc., pp. 33-40, 1988. 
[12] Treviso, C.H.G., De Freitas, O.C., and Farias V.J., "A ZVS-ZCS-PWM self resonant DC-DC Sepic converter," Industrial Electronics, Control and Instrumentation, Vol. 1, pp. 176 - 181,1994.

[13] V. Vorperian, "Quasi-Square-wave Converters: Topologies and Analysis," IEEE Trans. On Power Electronics, Vol. 3, No. 2, pp. 183-191,1998.

[14] V. Vorperian, R. Tymerski; F.C.Y Lee, "Equivalent circuit models for resonant and PWM switches," IEEE Trans. On Power Electronics, Vol. 4, pp. 205 - 214,1989.

[15] V. Vorperian, W.T. McLyman, "Analysis of a PWM-resonant converter," IEEE Trans. On Aerospace and Electronic Systems, Vol. 33, pp. 163 - 170, 1997.

[16] S.G. Trabert, R.W. Erickson, "Steady state analysis of the duty cycle controlled series resonant converter," IEEE Power Electronics Specialists Conf. Rec., pp. 545$556,1987$.

[17] OrCAD Pspice User's Guide.

[18] OrCAD Capture User's Guide.

[19] MicroSim Application Notes.

[20] M. Cotorogea , "Using analog behavioral modeling in PSpice for the implementation of subcircuit-models of power devices," IEEE International Power Electronics Congress, CIEP 98, pp. 158 - 163, 1998.

[21] http://mathworld.wolfram.com/SeriesExpansion.html.

[22] http://mathworld.wolfram.com/MaclaurinSeries.html.

[23] Daniel Zwillinger, "CRC Standard Mathematical Tables and Formulae," $31^{\text {st }}$ Edition, John Wiley, November 2002.

[24] M. Cotorogea, "Implementation of mathematical models of power devices for circuit simulation in Pspice," 6th Workshop on Computers in Power Electronics, 1998. pp. 17 - 22, 1998. 
[25] N.D Muhamad, A.J Shafie , "An approach to Pspice-aided control loop design of DC-DC converter systems," Power and Energy Conference, PECon, pp. 121 - 126, 2003.

[26] N.D Muhamad, A.J Shafie , "A PSPICE-based design of DC-DC converter systems," Power and Energy Conference, PECon, pp. 73 - 77, 2004.

[27] M.K Kazimierczuk, W.D Morse, "State-plane analysis of zero-current-switching resonant DC/DC power converters," IEEE Transactions on Power Electronics, Volume 4, pp 265 - 271, 1989.

[28] M.K Kazimierczuk, W.D Morse, "State-plane analysis of zero-voltage-switching resonant DC/DC power converters," IEEE Transactions on Power Electronics, Volume 24, pp 232-240, 1989.

[29] Naser Kutkut, C. Q. Lee and Issa Batarseh "A Generalized Program For Extracting the Control Characteristcs of Resonant Converters via State-Plane Diagram," IEEE. PP. 1236-1242, 1995.

[30] A. Alsharqawi, "Steady State Analysis of Softs Switching DC-DC and MAGAMP Forward Converters," Master Thesis, University of Central Florida,2002.

[31] A. Alsharqawi, I. Batarseh, "Generalized state-plane analysis of softswitching DC-DC converters," , IEEE International Symposium on Circuits and Systems (ISCAS), 2002.

[32] W. Al-Hoor, J. Abu-Qahouq and I. Batarseh, "Unified steady-state computer aided Model for soft-switching DC-DC converters," IEEE Applied Power Electronics Conference (APEC),2006. 